\title{
Six Degree of Freedom Vehicle Controller Design for the Operation of an Unmanned Underwater Vehicle in a Shallow Water Environment by
}

\author{
MICHAEL F. HAJOSY
}

B.S. Systems Engineering, U.S. Naval Academy (1985)

Submitted in partial fulfillment of the requirements for the dual degrees of

OCEAN ENGINEER

at the

MASSACHUSETTS INSTITUTE OF TECHNOLOGY and the WOODS HOLE OCEANOGRAPHIC INSTITUTION

and

\section{MASTER OF SCIENCE IN MECHANICAL ENGINEERING at the} MASSACHUSETTS INSTITUTE OF TECHNOLOGY

September 1994

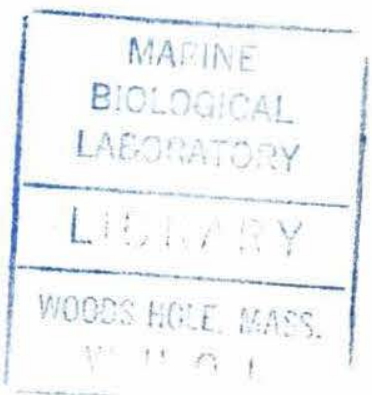

(C) Michael F. Hajosy, 1994. All rights reserved

The author hereby grants to MIT and WHOI permission to reproduce and to distribute publicly paper and electronics copiess of this thesis document in whole or in part.

Signature of Author

Joint Program in Uceanograpnic cuymu, $\overline{n g}$, Massachusetts Institute $\cap$ of Technology and Woods Hole Oceanographic Institution

Certified by

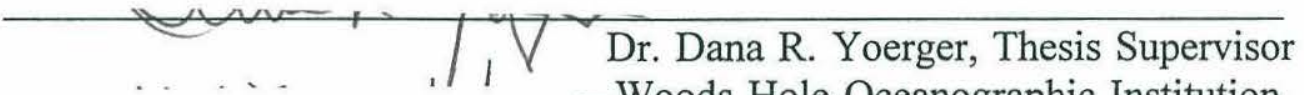
Woods Hole Oceanographic Institution

Certified by

$$
\vee v \checkmark \quad \checkmark \quad \text { ' 'Pfofessor Michael S. Triantafyllou, Thesis Reader }
$$

Department of Ocean Engineering, Massachusetts Institute of Technology

Certified by

Associáte Professor Jean-Jacques E. Slotine, Thesis Reader Department of Mechanical Engineering, Massachusetts Institute of Technology

Accepted by

Professor Arthur B. Baggeroer, Chairperson Joint Committee for Oceanographic Engineering Massachusetts Institute of Technology and the $\mathrm{W} \phi \mathrm{ds}$ (s)

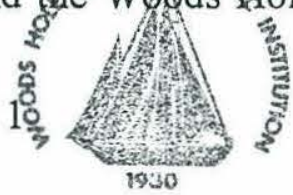




\title{
Six Degree of Freedom Vehicle Controller Design for the Operation of an Unmanned Underwater Vehicle in a Shallow Water Environment
}

\author{
by \\ Michael F. Hajosy \\ Submitted to the Massachusetts Institute of Technology and \\ Woods Hole Oceanographic Institution \\ Joint Program in Oceanographic Engineering \\ on August 5, 1994, in partial fulfillment of the \\ requirements for the degrees of \\ OCEAN ENGINEER \\ and \\ MASTER OF SCIENCE IN MECHANICAL ENGINEERING
}

\begin{abstract}
Closed loop control of an unmanned underwater vehicle (UUV) in the dynamically difficult environment of shallow water requires explicit consideration of the highly coupled nature of the governing non-linear equations of motion. This coupling between an UUV's six degrees of freedom (6 DOF) is particularly important when attempting complex maneuvers such as coordinated turns (e.g. simultaneous dive and heading change) or vehicle hovering in such an environment. Given the parameter and modelling uncertainties endemic to these equations of motion, then a robust 6 DOF sliding controller employing six-element vector sliding surfaces provides a framework in which satisfactory UUV control can be achieved in shallow water.

The vehicle equations of motion are developed and cast in a form that is amenable to non-linear sliding control design. A complete 6 DOF sliding controller with vector sliding surfaces is then formulated via a Lyapunov-like analysis. The sliding controller is then modified via a weighted least-squares approach to work with a particular UUV which has only 4 DOF control authority available. The modified controller is shown to work well for a variety of commanded UUV maneuvers in the presence of significant environmental disturbances and vehicle hydrodynamic parameter uncertainties via numerical simulation. Use of the signals generated by the controller are shown to be of utility in vehicle buoyancy control.
\end{abstract}

Thesis Supervisor: Dr. Dana R. Yoerger, Associate Scientist Woods Hole Oceanographic Institution 


\section{Acknowledgements}

As is the case for nearly all successfully completed endeavors in academics, a number of people besides the author were crucial and indispensable in the development of the ideas and research that went into this thesis. I cannot possibly individually thank each and every professor, instructor, teaching assistant, scientist, researcher, colleague and friend whose help - always provided generously and unreservedly - made this study possible; but I would like to cite and thank several whose assistance was of particular value:

- The U.S. Navy, whose financial assistance made this graduate study possible.

- Jean-Jacques Slotine and Dr. Fossen - the latter whom I have not had the pleasure of meeting - whose work was the basis for all that is this thesis.

- Mike Triantafyllou, who taught me how to think like an ocean engineer and was more than kind in research support.

- Dave Barrett, for his tremendous amount of guidance at the MIT Wave Tank.

- Dana Yoerger, my advisor, who provided the necessary support, guidance and freedom I needed these past two-plus years. I will always highly value the practical way he taught me to view engineering, a skill I know will greatly assist me in future endeavors.

- Chris Willy, whose advice and counsel has been invaluable.

- My parents, who always have been my biggest fans and cheerleaders.

- And most importantly, my immediate family - Cheryl, my wife and best friend, and Cassandra, my daughter - whose encouragement, understanding and love made it all possible.

In addition, the financial support of the Office of Naval Research under Contract No. N00014-90-J-1912 is gratefully acknowledged. 


\section{Author's Biographical Note}

Michael Francis Hajosy was born in 1963 in Hartford, Connecticut. While finishing high school in southern Maine, he received a congressional appointment to the United States Naval Academy. He graduated with distinction in 1985 with a B.S. in Systems Engineering and was commissioned an officer in the United States Navy.

After completing an twelve-month training program under the auspices of the Navy Nuclear Power Program, the author was assigned to the USS Mississippi (CGN-40) first serving as the Reactor Controls Division Officer in 1987. Significant billets while onboard included stints as the Electrical Officer and Assistant Engineer. He achieved the qualifications of Nuclear Engineer (Surface) and Surface Warfare Officer prior to being reassigned to serve as Division Director and instructor at the Navy Nuclear Power School for the Officer Chemistry, Materials Science and Radiological Fundamentals Division in 1990. While teaching at the Nuclear Power School the author also completed the coursework for a Masters degree in Engineering Management from the University of Central Florida (to be awarded Fall 1994).

Currently the author is a graduate student in the MIT/Woods Hole Oceanographic Institution Joint Program in Oceanographic Engineering. He lives in Cape Cod with his wife, Cheryl Lynne Godfrey, and 13/4 year old daughter, Cassandra Lynn. 


\section{Contents}

1. Introduction 12

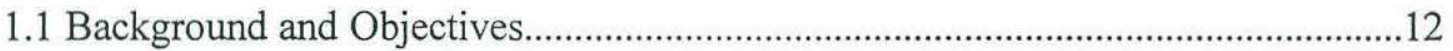

1.2 Thesis Outline

2. 6 DOF Rigid Body Equations of Motion 16

2.1 General Discussion...............................................................................................

2.2 Standard Notation and Reference Frames............................................................17

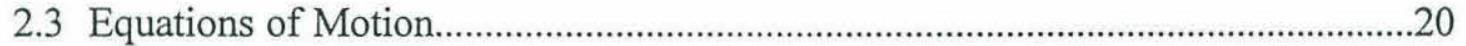

2.3.1 Notes on the Matrices in the Equations of Motion.......................................27

2.4 Hydrodynamic Coefficients...................................................................................28

3. 6 DOF Sliding Controller 30

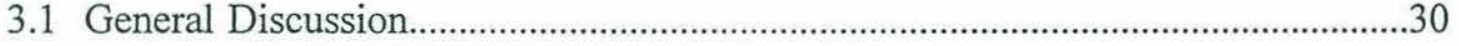

3.2 Scalar Sliding Control..........................................................................................

3.3 Vector Sliding Control..........................................................................................3

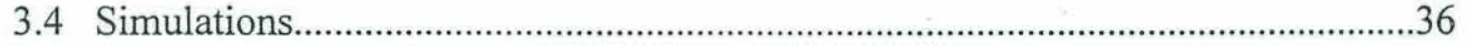

4. 4 DOF Implementation of the 6 DOF Sliding Controller 40

4.1 General Discussion..............................................................................................

4.2 6 DOF Commanded to 4 DOF Available Control......................................................41

4.2.1 Unweighted Least Squares..............................................................................42

4.2.2 Weighted Least Squares................................................................................43

4.2.3 Justification for the Weighted LeastSquaresMethod................................45 


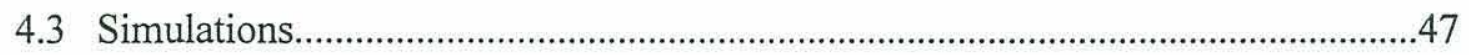

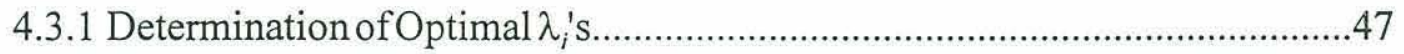

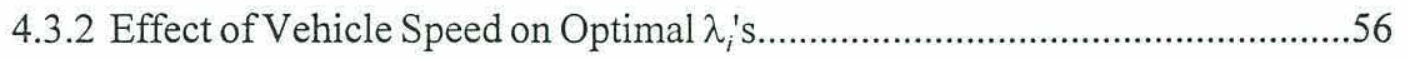

4.3.3 Effect of Trajectory Severity on Optimal $\lambda_{i}$ 's................................................61

4.3.4 Vehicle Roll Capability....................................................................................66

4.3.5 Vehicle Coordinated Maneuvering Response..................................................71

5. Related Topics to the 6 DOF Sliding Controller 86

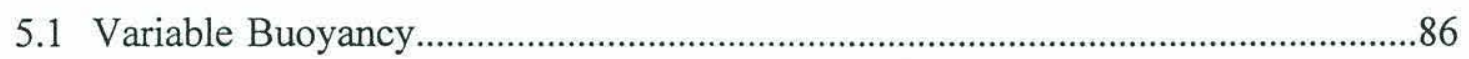

5.2 Disturbance Response.........................................................................................91

6. Conclusion $\quad 100$

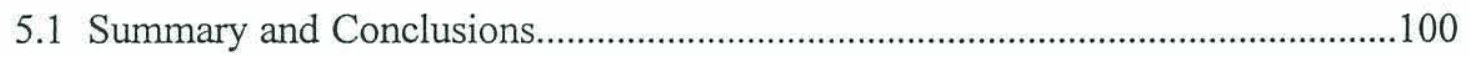

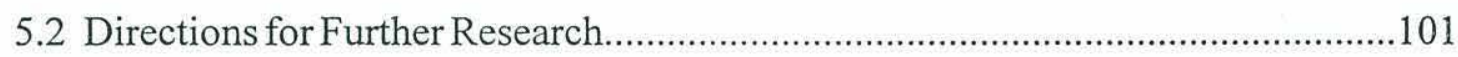

$\begin{array}{ll}\text { References } & 103\end{array}$

$\begin{array}{ll}\text { Appendix I } & 105\end{array}$

21UUV Body and Hydrodynamic Coefficients................................................................105

$\begin{array}{ll}\text { Appendix II } & 106\end{array}$

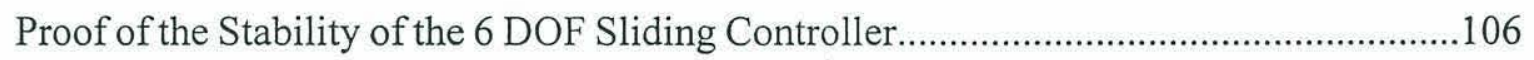




\section{List of Figures}

$1.1 \quad 21$ UUV Profile $\ldots \ldots \ldots \ldots \ldots \ldots \ldots \ldots \ldots \ldots \ldots \ldots \ldots \ldots$

3.1 Comparison of Actual vs. Desired Vehicle Response During a Coordinate Maneuver Assuming Full 6 DOF Control Authority Available . . . . . . . . 39

4.1 Comparison of Sternplane Response in a Commanded Dive for Pitch Weights

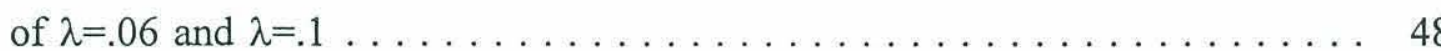

4.2 Comparison of Depth and Depth Rate Responses in a Commanded Dive for

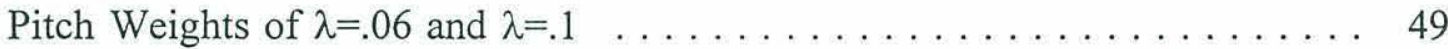

4.3 Comparison of Pitch and Pitch Rate Responses in a Commanded Dive for Pitch

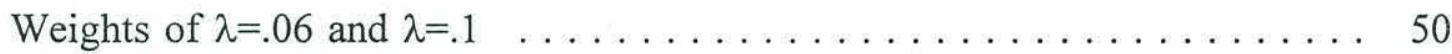

4.4 Comparison of Forward Speed and Thrust Responses in a Commanded Dive for

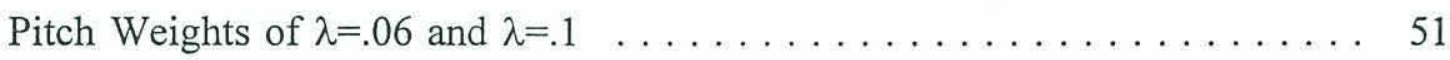

4.5 Comparison of Sternplane Response in a Commanded Dive for Pitch Weights

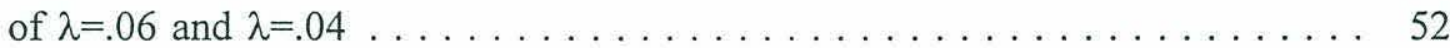

4.6 Comparison of Depth and Depth Rate Responses in a Commanded Dive for

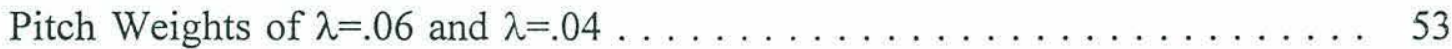

4.7 Comparison of Pitch and Pitch Rate Responses in a Commanded Dive for

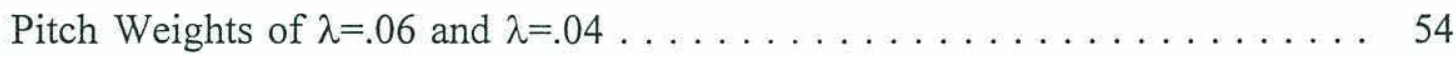

4.8 Comparison of Forward Speed and Thrust Responses in a Commanded Dive for

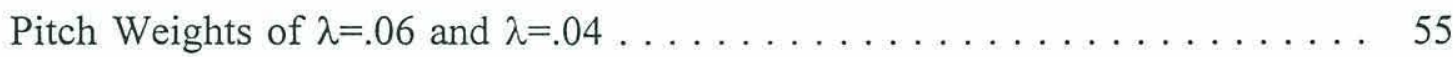

4.9 Comparison of Sternplane Response in a Commanded Dive at 5 and 8 Knots Nominal Speed for a Pitch Weighing of $\lambda=.06 \ldots \ldots \ldots \ldots \ldots$

4.10 Comparison of Depth and Depth Rate Responses in a Commanded Dive at 5 and 8 Knots Nominal Speed for a Pitch Weighing of $\lambda=.06$ 
4.11 Comparison of Pitch and Pitch Rate Responses in a Commanded Dive at 5 and 8 Knots Nominal Speed for a Pitch Weighing of $\lambda=.06 \ldots \ldots \ldots \ldots \ldots$

4.12 Comparison of Forward Speed and Thrust Responses in a Commanded Dive at 5 and 8 Knots Nominal Speed for a Pitch Weighing of $\lambda=.06 \ldots \ldots \ldots 60$

4.13 Comparison of Sternplane Response in Commanded Dives of 2.5 and 5 Meters at 5 Knots Nominal Speed for a Pitch Weighing of $\lambda=.06 \ldots \ldots \ldots \ldots 6$

4.14 Comparison of Depth and Depth Rate Responses in Commanded Dives of 2.5 and 5 Meters at 5 Knots Nominal Speed for a Pitch Weighing of $\lambda=.06 \ldots 63$

4.15 Comparison of Pitch and Pitch Rate Responses in Commanded Dives of 2.5 and 5 Meters at 5 Knots Nominal Speed for a Pitch Weighing of $\lambda=.06 \quad \ldots .64$

4.16 Comparison of Forward Speed and Thrust Responses in Commanded Dives of 2.5 and 5 Meters at 5 Knots Nominal Speed for a Pitch Weighing of $\lambda=.06$. .

4.17 Roll and Aileron Responses for a Commanded Counter-Clockwise Roll of 10

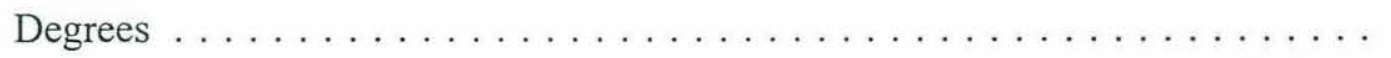

4.18 Depth and Pitch Angle Responses for a Commanded Counter-Clockwise Roll of 10 Degrees . . . . . . . . . . . . . . . . . . . . 68

4.19 Trackline and Yaw Angle Responses for a Commanded Counter-Clockwise Roll of 10 Degrees . . . . . . . . . . . . . . . . . . . . . . 69

4.20 Sternplane and Rudder Responses for a Commanded Counter-Clockwise Roll of

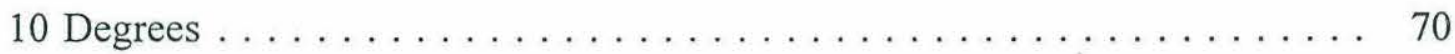

4.21 Depth and Depth Rate for a Coordinated Dive and Trackline Maneuver with a

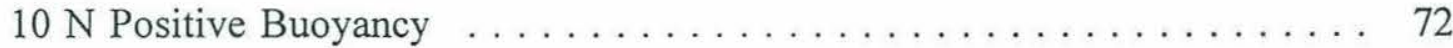

4.22 Trackline and Heading Rate for a Coordinated Dive and Trackline Maneuver with a $10 \mathrm{~N}$ Positive Buoyancy $\ldots \ldots \ldots \ldots \ldots \ldots \ldots \ldots \ldots \ldots$

4.23 Sternplane and Rudder Responses for a Coordinated Dive and Trackline Maneuver with a $10 \mathrm{~N}$ Positive Buoyancy . . . . . . . . . . . 74

4.24 Pitch and Pitch Rate for a Coordinated Dive and Trackline Maneuver with a 10

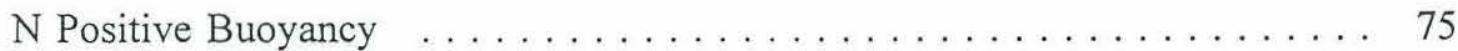

4.25 Yaw and Yaw Rate for a Coordinated Dive and Trackline Maneuver with a 10

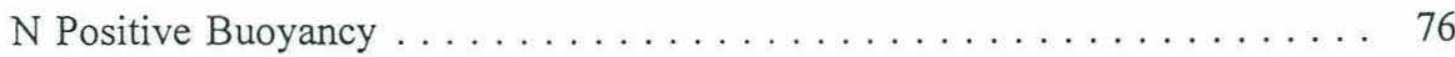


4.26 Forward Speed and Thrust for a Coordinated Dive and Trackline Maneuver with a $10 \mathrm{~N}$ Positive Buoyancy $\ldots \ldots \ldots \ldots \ldots \ldots \ldots \ldots \ldots \ldots$

4.27 Roll and Aileron Angles for a Coordinated Dive and Trackline Maneuver with

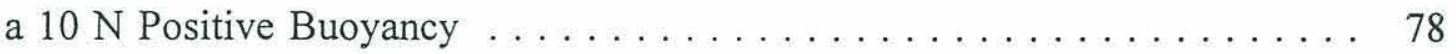

4.28 Depth and Depth Rate for a Coordinated Rise and Trackline Maneuver with a $10 \mathrm{~N}$ Positive Buoyancy . . . . . . . . . . . . . . . . . . . 79

4.29 Trackline and Heading Rate for a Coordinated Rise and Trackline Maneuver with a $10 \mathrm{~N}$ Positive Buoyancy $\ldots \ldots \ldots \ldots \ldots \ldots \ldots \ldots \ldots . . \ldots$

4.30 Sternplane and Rudder Responses for a Coordinated Rise and Trackline Maneuver with a $10 \mathrm{~N}$ Positive Buoyancy $\ldots \ldots \ldots \ldots \ldots \ldots \ldots \ldots . \ldots 1$

4.31 Pitch and Pitch Rate for a Coordinated Rise and Trackline Maneuver with a 10 N Positive Buoyancy . . . . . . . . . . . . . . . . . . . 82

4.32 Yaw and Yaw Rate for a Coordinated Rise and Trackline Maneuver with a 10 N Positive Buoyancy . . . . . . . . . . . . . . . . . . .

4.33 Forward Speed and Thrust for a Coordinated Rise and Trackline Maneuver with a $10 \mathrm{~N}$ Positive Buoyancy $\ldots \ldots \ldots \ldots \ldots \ldots \ldots \ldots \ldots . \ldots . \ldots$

4.34 Roll and Aileron Angles for a Coordinated Rise and Trackline Maneuver with a $10 \mathrm{~N}$ Positive Buoyancy $\ldots \ldots \ldots \ldots \ldots \ldots \ldots \ldots \ldots \ldots$

5.1 6 DOF Sliding Controller Generated Earth-Referenced Z Force and M Moment

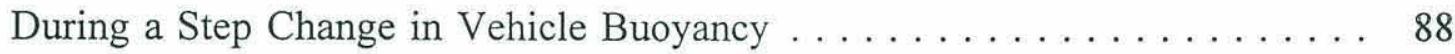

5.2 Sternplane Response During a Step Change in Vehicle Buoyancy . . . . . . . 89

5.3 Excursions from Nominal Depth and Pitch Angle During a Step Change in

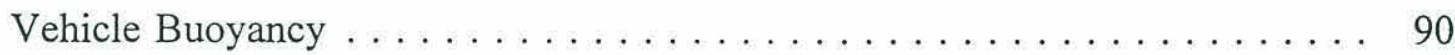

5.4 Depth and Depth Rate for a Coordinated Dive and Trackline Maneuver with a $10 \mathrm{~N}$ Positive Buoyancy in the Presence of Simulated Wave Disturbances ... 93

5.5 Trackline and Heading Rate for a Coordinated Dive and Trackline Maneuver with a $10 \mathrm{~N}$ Positive Buoyancy in the Presence of Simulated Wave Disturbances . . . . . . . . . . . . . . . . . . . . . . . . 94

5.6 Sternplane and Rudder Responses for a Coordinated Dive and Trackline Maneuver with a $10 \mathrm{~N}$ Positive Buoyancy in the Presence of Simulated Wave 
Disturbances ............................ 95

5.7 Pitch and Pitch Rate for a Coordinated Dive and Trackline Maneuver with a 10 N Positive Buoyancy in the Presence of Simulated Wave Disturbances ... . 96

5.8 Yaw and Yaw Rate for a Coordinated Dive and Trackline Maneuver with a 10 N Positive Buoyancy in the Presence of Simulated Wave Disturbances . . . . 97

5.9 Forward Speed and Thrust for a Coordinated Dive and Trackline Maneuver with a $10 \mathrm{~N}$ Positive Buoyancy in the Presence of Simulated Wave Disturbances . . . . . . . . . . . . . . . . . . . . . . . 98

5.10 Roll and Aileron Angles for a Coordinated Dive and Trackline Maneuver with a 10 N Positive Buoyancy in the Presence of Simulated Wave Disturbances . . 99 


\section{List of Tables}

2.1 Standard Underwater Vehicle Notation .............................................................18 


\section{Chapter 1}

\section{Introduction}

\subsection{Background and Objectives}

Unmanned Underwater Vehicles (UUV's) have become increasingly popular tools for use in a number of ocean research and industrial applications. As the missions for these UUV's expand in scope, their capabilities must also increase - not only in terms of improved vehicle control and manueverablity but also with regards to satisfactory performance in challenging environments.

For instance, consider the vehicle of figure 1.1 which is currently under development at the Naval Undersea Warfare Center (NUWC) in Newport, Rhode Island. This 301 inch long, 21 inch in diameter torpedo shaped vehicle - known as the 21UUV - is being designed so as to have the capability to perform sophisicated maneuvers in the dynamically-challenging regime of shallow water.

Needless to say, the controller which enables this UUV to attain such capability must be fairly sophisticated itself. Not only must the control algorithm be robust to the uncertain and changing values of the hydrodynamic coefficients which define the vehicle 


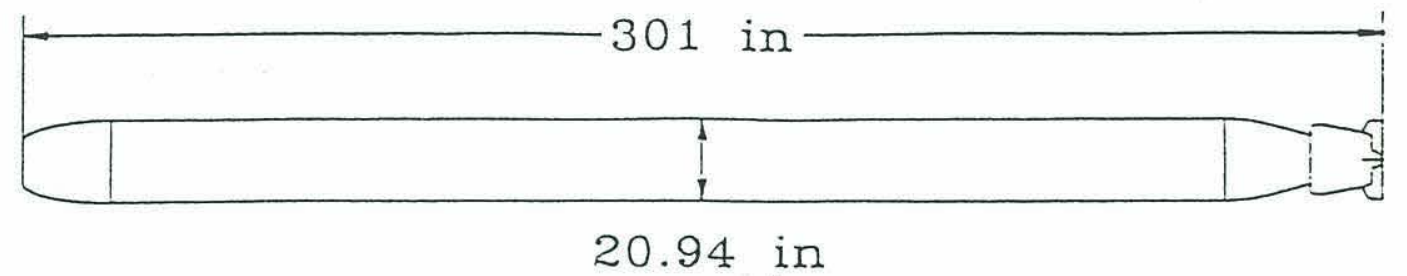

\section{Figure 1.1 2lUUV}

non-linear equations of motion, it must be able to account for the dynamic coupling that will occur between the vehicle's various degrees of freedom as complex maneuvers are undertaken and/or the wave disturbances are significant - as they would be in shallowwater.

Typically, the coupling in these non-linear equations of motion are subjected to various simplifying assumptions so that particular vehicle design objectives can be satisfied (e.g. decoupled speed control, inner pitch/outer depth loop control, etc.). Such simplifying assumptions also allow the use of sliding controllers of first-order (i.e. scalar sliding surfaces) - a non-linear robust control methodology which has proven well-suited to control in the ocean environment.

As alluded to, however, more demanding underwater vehicle maneuvers such as hovering require careful consideration of the coupling inherent to the equations of motion. Likewise, the operation of such a vehicle in the dynamically difficult regime of shallow water necessitates a thorough accounting of this coupling if high performance is to be 
acheived. As will be demonstrated, a complete 6 DOF sliding controller (i.e. a 6 element vector sliding surface) provides an effective framework for handling these situations.

This study focused on the development of a six degree of freedom (6 DOF) robust sliding controller to be used on a variety of UUV designs in dynamically difficult regimes like shallow water as well as for complicated maneuvering such as hovering. As few practical UUV's have the 6 or more controllable degrees of freedom a method is presented which modifies the 6 DOF control signal so that a 4 DOF control authority-available vehicle such as the $21 \mathrm{UUV}$ can reap the benefits of a complete, coupled non-linear controller.

A side benefit of the 6 DOF sliding controller is that it produces a signal which is ideal for use in buoyancy control systems. In addition, mention is made as to how disturbance adaptation/control might be formulated with the 6 DOF controller.

\subsection{Thesis Outline}

Chapter 2 details the development of the general non-linear and coupled 6 DOF UUV equations of motion. These equations are formulated with respect to two reference frames - vehicle-fixed and earth (inertial)-fixed - and in such a format that readily lends itself to non-linear controller design techniques as outlined in chapter 3. Key matrix relationships that characterize resultant UUV equations of motion are described, as these form the basis of the robust non-linear sliding controller. The sources and values of the various body and hydrodynamic coefficients for the $21 \mathrm{UUV}$ of figure 1.1 which will be used in the numerical simulations of Chapters 3,4 and 5 are also summarized. 
Chapter 3 presents the formulation of the complete 6 DOF robust sliding controller which is shown to be stable - assuming full 6 DOF control authority - from any vehicle configuration except at \pm 90 degrees of pitch.

As full 6 DOF control authority is usually not available, an attempt is made in Chapter 4 to develop a method to modify this sliding controller so that lesser degree of freedom vehicles (in terms of available control authority) would perform satisfactorily. As is demonstrated with the 4 DOF $21 \mathrm{UUV}$, this method - weighted least squares - is based heavily on the non-minimum phase relationship between pitch/depth and yaw/trackline. Extensive simulations are presented to evaluate the effectiveness and validity of this method.

Chapter 5 briefly delves into two areas related to the controller development of the previous chapters - vehicle buoyancy control and wave disturbance rejection/control. The earth-referenced vehicle control signal generated by the sliding controller of Chapter 3 is shown by computer simulations on the 21UUV to be of great utility as an input to a variable buoyancy control system. Vehicle response in a wave field is simulated.

Chapter 6 summarizes the results of this thesis and suggests directions for further investigation. 


\section{Chapter 2}

\section{DOF Rigid Body Equations of Motion}

\subsection{General Discussion}

In this chapter the $6 \mathrm{DOF}$ rigid body underwater vehicle equations of motion are developed with the primary emphasis being the identification of a particularly compact and convenient way to express them for exploitation by nonlinear sliding controller design techniques as outlined in chapter 3.

Section 2.2 introduces the standard notation and conventions utilized in underwater vehicle design. The two reference frames commonly used to describe the motion of and forces/moments on the vehicle - body-fixed and earth-fixed (inertial) - are introduced as well. It is of considerable importance to both the development of the equations of motion and to the subsequent $6 \mathrm{DOF}$ sliding controller formulation that the two reference frames and the relationship between them are thoroughly understood. As might be expected from physical intuition, the vehicle linear and angular velocities and corresponding accelerations along with the vehicle external forces and moments are best described in the body-fixed frame; whereas, the vehicle's linear and angular positions are best described 
in an inertial reference frame in which commanded trajectories are typically expressed. For small angle, non-coupled vehicle maneuvers the distinction between the reference frames is slight and can be safely ignored. However, for more vigorous, coupled maneuvers (e.g. a coupled dive/trackline commanded trajectory or hovering) and/or for operation in the more dynamically-challenging environment of shallow water, this distinction must be preserved.

With this last point in mind, the full 6 DOF underwater vehicle equations of motion are derived in the body-fixed reference frame in section 2.3. The resultant equations are parameterized in a specific matrix form which turns out to be particularly amenable to sliding controller design. The full 6 DOF earth-fixed and body-fixed nonlinear UUV equations of motion are summarized in (2.26)-(2.28).

The final section of this chapter summarizes the values of the hydrodynamic coefficients used in these equations of motion and how they were determined.

\subsection{Standard Notation and Reference Frames}

For (rigid body) underwater vehicles which operate in a three dimensional space, it is necessary to describe the position/orientation and motion/rotation of the vehicle by six independent coordinates - hence, the term six degree of freedom (6 DOF). Typically these coordinates are chosen to correspond to the position and orientation and their time derivatives with respect to some set or sets of mutually orthogonal coordinate axes (e.g. $x-, y-, z$-axes) fixed to some arbitrary origin which defines a reference frame. Likewise, the forces/moments acting on or produced by the vehicle can be referenced to a set of 
coordinate axes. Standard (SNAME [1950]) notation is used in this study to describe the aforementioned 6 DOF quantities and is summarized in Table 2.1.

\begin{tabular}{||c|c|c|c|c||}
\hline DOF & Motions & $\begin{array}{c}\text { Forces and } \\
\text { Moments }\end{array}$ & $\begin{array}{c}\text { Linear and } \\
\text { Angular Vel. }\end{array}$ & $\begin{array}{c}\text { Positions and } \\
\text { Euler Angles }\end{array}$ \\
\hline 1 & surge & $\mathrm{X}$ & $\mathrm{u}$ & $\mathrm{x}$ \\
\hline 2 & sway & $\mathrm{Y}$ & $\mathrm{v}$ & $\mathrm{y}$ \\
\hline 3 & heave & $\mathrm{Z}$ & $\mathrm{w}$ & $\mathrm{z}$ \\
\hline 4 & roll & $\mathrm{K}$ & $\mathrm{p}$ & $\phi$ \\
\hline 5 & pitch & $\mathrm{M}$ & $\mathrm{q}$ & $\theta$ \\
\hline 6 & yaw & $\mathrm{N}$ & $\mathrm{r}$ & $\psi$ \\
\hline
\end{tabular}

Table 2.1 - Standard Underwater Vehicle Notation

Note that by convention for underwater vehicles, the positive $\mathrm{x}$ direction is taken as forward, the positive $\mathrm{y}$ direction is taken as to the right, the positive $\mathrm{z}$ direction is taken as down, and the right-hand rule applies for angles. It is convenient to group the linear and angular velocities as a vector quantity $v$, where:

$$
\mathbf{v}=\left[\begin{array}{llllll}
u & v & w & p & q & r
\end{array}\right]^{T}
$$

the positions and Euler angles as a vector quantity $\eta$, where:

$$
\eta=\left[\begin{array}{llllll}
x & y & z & \phi & \theta & \psi
\end{array}\right]^{T}
$$

and the forces and moments as a vector quantity $\tau$, where:

$$
\tau=\left[\begin{array}{llllll}
X & Y & Z & K & M & N
\end{array}\right]^{T}
$$

As previously indicated, it is not necessary to choose the same reference frame for the three quantities of (2.1)-(2.3). In fact, it is customary and makes physical sense to 
refer $v$ and $\tau$ to the vehicle-fixed (body) reference frame and to refer $\eta$ to an inertial reference frame. Note that choosing these particular frames of reference directly influences the form of the $6 \mathrm{DOF}$ equations of motion that are to be developed. The body-fixed reference frame is chosen to coincide with the vehicle's principle axes of inertia. The earth is chosen as the inertial reference frame since underwater vehicles travel at low enough speeds so that the acceleration of points on the surface of the earth can be neglected.

As it will be necessary to convert from one reference frame to the other, a relationship between $v$ and $\eta$ for the particular positive axis directions that have been defined can be shown to be (see Fossen [1994]):

$$
\dot{\eta}=J(\eta) v
$$

where:

$$
J(\eta)=\left[\begin{array}{cc}
J_{1}\left(\eta_{2}\right) & 0 \\
0 & J_{2}\left(\eta_{2}\right)
\end{array}\right]
$$

and $(s=\sin , c=\cos , t=\tan )$ :

$$
J_{1}\left(\boldsymbol{\eta}_{2}\right)=\left[\begin{array}{ccc}
c \psi c \theta & -s \psi c \phi+c \psi s \theta s \phi & s \psi s \phi+c \psi c \phi s \theta \\
s \psi c \theta & c \psi c \phi+s \phi s \theta s \psi & -c \psi s \phi+s \theta s \psi c \phi \\
-s \theta & c \theta s \phi & c \theta c \phi
\end{array}\right]
$$

and: 


$$
J_{2}\left(\eta_{2}\right)=\left[\begin{array}{ccc}
1 & s \phi t \theta & c \phi t \theta \\
0 & c \phi & -s \phi \\
0 & s \phi / c \theta & c \phi / c \theta
\end{array}\right]
$$

Specifically, (2.5) represents a series of three rotations - about the yaw angle then the pitch angle then the roll angle - to get from the vehicle-fixed reference frame to the earthfixed reference frame.

Note that (2.7) is undefined for pitch angles of \pm 90 degrees. To overcome this problem, one can use a quaternion representation to describe the coordinate axis rotations implied by (2.5) - see Fossen [1994] - or one could define a second body-fixed reference frame (different from the first) and switch to its use when approaching the singular orientations of the first reference frame. Fortunately, vehicle metacentric restoring forces and typical commanded vehicle trajectories preclude operation near these singularities so (2.4)-(2.7) are considered adequate for the purposes of this study.

\subsection{Equations of Motion}

Derivation of the underwater vehicle equations of motion has been extensively studied over the years and can be accomplished by a number of techniques (e.g. NewtonEuler, Kirchoff, Lagrangian). This paper will briefly present the results of a NewtonEuler formulation so that the final form of the equations of motion and that some assumptions utilized in the sliding controller design are made clear. The interested reader is encouraged to consult a suitable hydrodynamic treatise (e.g. Fossen [1994]) for a more complete treatment of this subject. 
It is extremely desirable to derive these equations of motion in a body-fixed reference frame so that the hydrodynamic and kinematic forces and moments remain constant despite changes in the vehicle's orientation with respect to the earth-fixed reference frame. From Newton's Second Law:

$$
m \dot{v}=f
$$

where:

$$
\begin{aligned}
m & =\text { vehicle mass } \\
\dot{v} & =\text { acceleration vector } \\
f & =\text { force vector }
\end{aligned}
$$

it is apparent that the decision to formulate the equations of motion in the body-fixed reference frame trades off complexity in the acceleration terms for relative simplicity in the force terms. With this in mind, conservation of linear momentum for a rigid body yields (Euler's First Axiom of Newton's Second Law):

$$
m\left(\dot{v}_{o}+\boldsymbol{\omega} \times v_{o}+\dot{\boldsymbol{\omega}} \times \boldsymbol{r}_{G}+\boldsymbol{\omega} \times\left(\boldsymbol{\omega} \times \boldsymbol{r}_{G}\right)\right)=f_{o}
$$

where:

$$
\begin{aligned}
m & =\text { vehicle mass } \\
\boldsymbol{\omega} & =\text { angular velocity vector } \\
\dot{\omega} & =\text { angular accleration vector } \\
v_{o} & =\text { vehicle body referenced vel. } \\
\dot{v}_{o} & =\text { vehicle body referenced acc. } \\
r_{G} & =\text { vector origin o } \rightarrow \text { center gravity } \mathrm{G} \\
f_{o} & =\text { external force vector }
\end{aligned}
$$

In a similar manner, conservation of angular momentum yields: 


$$
I_{o} \dot{\boldsymbol{\omega}}+\omega \times\left(I_{o} \boldsymbol{\omega}\right)+m r_{G} \times\left(\dot{v}_{o}+\boldsymbol{\omega} \times v_{o}\right)=\boldsymbol{m}_{o}
$$

where:

$$
\begin{aligned}
I_{o} & =\text { body inertia tensor } \\
m_{0} & =\text { applied moment vector }
\end{aligned}
$$

Combining (2.10) and (2.12) results in (after some judicious rearrangement of terms):

$$
M_{R B} \dot{v}+C_{R B}(v) v=\tau_{R B}
$$

where, assuming the $\mathrm{x}-, \mathrm{y}$ - and $\mathrm{z}$ - body axes correspond to the longitudinal, lateral and normal symmetry axes of the vehicle (so all cross-inertia terms are zero - usually an excellent supposition and especially so for the 21UUV of figure 1.1) and assuming the lateral center of gravity corresponds to the lateral axis origin $\left(y_{G}\right.$ equals zero - again, an excellent assumption):

$$
M_{R B}=\left[\begin{array}{cccccc}
m & 0 & 0 & 0 & m z_{g} & 0 \\
0 & m & 0 & -m z_{g} & 0 & m x_{g} \\
0 & 0 & m & 0 & -m x_{g} & 0 \\
0 & -m z_{g} & 0 & I_{x} & 0 & 0 \\
m z_{g} & 0 & -m x_{g} & 0 & I_{y} & 0 \\
0 & m x_{g} & 0 & 0 & 0 & I_{z}
\end{array}\right]
$$

and: 


$$
C_{R B}(v)=\left[\begin{array}{cccccc}
0 & 0 & 0 & m z_{G} r & -m\left(x_{G} q-w\right) & -m\left(x_{G} r+v\right) \\
0 & 0 & 0 & -m w & m\left(z_{G} r+x_{G} p\right) & m u \\
0 & 0 & 0 & -m\left(z_{G} p-v\right) & -m\left(z_{G} q+u\right) & m x_{G} p \\
-m z_{G} r & m w & m\left(z_{G} p-v\right) & 0 & I_{z} r & -I_{y} q \\
m\left(x_{G} q-w\right) & -m\left(z_{G} r+x_{G} p\right) & m\left(z_{G} q+u\right) & -I_{z} r & 0 & I_{\not \supset} p \\
m\left(x_{G} r+v\right) & -m u & -m x_{G} p & I_{y} q & -I_{\not} p & 0
\end{array}\right]
$$

and:

$$
\tau_{R B}=\left[\begin{array}{llllll}
X & Y & Z & K & M & N
\end{array}\right]^{T}
$$

Note, that the rigid-body inertia matrix $M_{R B}$ is symmetric, positive-definite (i.e. $\left.\boldsymbol{M}_{R B}=\left(\boldsymbol{M}_{R B}\right)^{\mathrm{T}}>0\right)$ and time-invariant, whereas the rigid-body Coriolis and centripetal matrix $C_{R B}(v)$ is skew-symmetric (i.e. $\left.C_{R B}(v)=-\left(C_{R B}(v)\right)^{T} \forall v \in \Re^{6}\right)$. Both facts will be useful in the 6 DOF sliding controller design.

The generalized vector of external forces and moments $\tau_{R B}$ can be expressed as the sum of radiation induced forces $\tau_{R}$ (e.g. added mass, potential damping and restoring forces), viscous damping forces $\tau_{V}$, environmental forces (such as waves and current) and propulsion forces $\tau$. Ignoring the latter forces (for now) and adopting the treatment of $\tau_{R}$ and $\tau_{V}$ suggested by Fossen [1991] yields:

$$
\begin{array}{ccc}
\tau_{R}=-M_{A} \dot{v}-C_{A}(v) v & -D_{P}(v) v & -g(\eta) \\
\text { added } & \text { potential } & \text { restoring } \\
\text { mass } & \text { damping } & \text { forces }
\end{array}
$$

and:

$$
\tau_{V}=-D_{V}(v) v
$$

Assuming the vehicle has bottom/top and port/starboard symmetry (which the 
21UUV of figure 1.1 has), the added inertia matrix $M_{A}$ and the hydrodynamic Coriolis and centripetal matrix $C_{A}(v)$ can be written as (see Faltinsen [1990]):

$$
M_{A}=-\left[\begin{array}{cccccc}
X_{\dot{u}} & 0 & 0 & 0 & 0 & 0 \\
0 & Y_{\dot{v}} & 0 & 0 & 0 & Y_{\dot{r}} \\
0 & 0 & Z_{\dot{w}} & 0 & Z_{\dot{q}} & 0 \\
0 & 0 & 0 & K_{\dot{p}} & 0 & 0 \\
0 & 0 & M_{\dot{w}} & 0 & M_{\dot{q}} & 0 \\
0 & N_{\dot{v}} & 0 & 0 & 0 & N_{\dot{r}}
\end{array}\right]
$$

and:

$$
C_{A}(v)=\left[\begin{array}{cccccc}
0 & 0 & 0 & 0 & C_{A}^{15} & C_{A}^{15} \\
0 & 0 & 0 & C_{A}^{24} & 0 & C_{A}^{26} \\
0 & 0 & 0 & C_{A}^{34} & C_{A}^{35} & 0 \\
0 & -C_{A}^{24} & -C_{A}^{34} & 0 & C_{A}^{45} & C_{A}^{46} \\
-C_{A}^{15} & 0 & -C_{A}^{35} & -C_{A}^{45} & 0 & C_{A}^{56} \\
-C_{A}^{16} & -C_{A}^{26} & 0 & -C_{A}^{46} & -C_{A}^{56} & 0
\end{array}\right]
$$

where:

$$
\begin{array}{cl}
C_{A}^{15}=-Z_{\dot{w}} w-Z_{\dot{q}} q & C_{A}^{16}=Y_{\dot{v}} \nu+Y_{\dot{r}} r \\
C_{A}^{24}=Z_{\dot{w}} w+Z_{\dot{q}} q \quad C_{A}^{26}=-X_{\dot{u}} u \\
C_{A}^{34}=-Y_{\dot{v}} v-Y_{\dot{r}} r \quad C_{A}^{35}=X_{\dot{u}} u \\
C_{A}^{45}=-Y_{\dot{r}} \nu-N_{\dot{r}} r \quad C_{A}^{46}=Z_{\dot{q}} w+M_{\dot{q}} q \\
C_{A}^{56}=-K_{\dot{p}} p
\end{array}
$$

The hydrodynamic coefficients in (2.20) and (2.22) follow the standard notation of SNAME [1950]. Refer to Appendix I for further details. 
Note that for a submerged rigid-body vehicle moving in still water under the assumption of an ideal fluid, $\boldsymbol{M}_{A}$ is always positive definite (same condition as $\boldsymbol{M}_{R B}$ ); a condition which is also almost invariably met for submerged vehicles in real fluids (see Wendel [1956] or Newman [1977], for example). Similar properties hold for $C_{A}(v)$ - i.e. for nearly all real fluid conditions $C_{A}(v)$ is skew-symmetric (like $C_{R B}(v)$ ) - see Sagatun and Fossen [1991].

The potential damping term $D_{p}(v)$ is usually quite small for underwater vehiclesespecially for operations away from the surface wave zone. Consequently, these effects are ignored for this analysis. Even if this term was numerically significant, it is dissipative by definition which implies that $\boldsymbol{D}_{p}(v)$ is positive definite (i.e. $v^{\mathrm{T}} \boldsymbol{D}_{p}(v) v>0$ $\forall v \neq 0)$ - an important fact in the development of the 6 DOF sliding controller.

By the same dissipative nature of the linear skin friction and quadratic vortexshedding drag forces which constitute the viscous damping matrix $D_{v}(v)$, it also is always positive definite. The following form for this matrix is chosen to be:

$$
D_{V}(\boldsymbol{v})=-\left[\begin{array}{cccccc}
X_{u|u|}|u| & X_{v r} r & X_{w q} q & 0 & 0 & 0 \\
0 & Y_{v} & 0 & 0 & 0 & Y_{r} \\
0 & 0 & Z_{w} & 0 & Z_{q} & 0 \\
0 & 0 & 0 & K_{p} & 0 & 0 \\
0 & 0 & M_{w} & 0 & M_{q} & 0 \\
0 & N_{v} & 0 & 0 & 0 & N_{r}
\end{array}\right]
$$

The terms on the diagonal of (2.23) represent the drag generated along the $i$ th DOF for motion in the $i$ th DOF; the off-diagonal $\mathrm{X}$ coefficients $-\mathrm{X}_{\mathrm{vr}}$ and $\mathrm{X}_{\mathrm{wq}}$ - represent the loss in forward speed generated in a turn and in a depth change, respectively; and the 
remaining off-diagonal terms capture the coplanar drag generated in turns and/or depth changes. Coplanar refers to coupled DOF's like pitch-depth which naturally occur together in typical vehicle maneuvers.

Other terms could be inserted into (2.23) such as $\mathrm{K}_{\mathrm{wr}} \mathrm{r}$ (a non-coplanar coefficient) - however, (2.23) characterizes the vast majority of the dissipative forces encountered during typical maneuvers for vehicles such as the 21UUV of figure 1.1 (see Humphreys [1989]). Those terms that are ignored do not affect the positive definite quality of the matrix since damping forces by definition are dissipative.

The restoring force vector $g(\eta)$ consists of the gravitational forces acting at the center of gravity $\left(x_{G}=\left[x_{G}, y_{G}, z_{G}\right]^{\mathrm{T}}\right)$ and the buoyant forces acting at the center of buoyancy $\left(x_{B}=\left[x_{B}, y_{B}, z_{B}\right]^{\mathrm{T}}\right)$ and has the form (assuming both $y_{G}$ and $y_{B}$ zero):

$$
g(\eta)=\left[\begin{array}{c}
(W-B) s \theta \\
-(W-B) c \theta s \phi \\
-(W-B) c \theta c \phi \\
\left(z_{G} W-z_{B} B\right) c \theta s \phi \\
\left(z_{G} W-z_{B} B\right) s \theta+\left(x_{G} W-x_{B} B\right) c \theta c \phi \\
-\left(x_{G} W-x_{B} B\right) c \theta s \phi
\end{array}\right]
$$

Defining:

$$
M=M_{R B}+M_{A} ; C(v)=C(v)_{R B}+C_{A}(v) ; D(v)=D_{P}(v)+D_{v}(v)
$$

Then the combination of equation (2.14) and (2.25) yields the body-fixed 6 DOF representation of the non-linear equations of motion for underwater vehicles: 


$$
M \dot{v}+C(v) v+D(v) v+g(\eta)=\tau
$$

To obtain the earth-fixed $6 \mathrm{DOF}$ representation of the non-linear equations of motion for underwater vehicles, the transformation of (2.4) must be applied to (2.26) yielding:

$$
M_{\eta}(\eta) \ddot{\eta}+C_{\eta}(v, \eta) \dot{\eta}+D_{\eta}(v, \eta) \dot{\eta}+g_{\eta}(\eta)=\tau_{\eta}
$$

where:

$$
\begin{aligned}
M_{\eta}(\eta) & =J^{-T}(\eta) M J^{-1}(\eta) \\
C_{\eta}(\boldsymbol{v}, \boldsymbol{\eta}) & =J^{-T}(\eta)\left[C(v)-M J^{-1}(\eta) \dot{J}(\eta)\right] J^{-1}(\eta) \\
D_{\eta}(v, \eta) & =J^{-T}(\eta) D(v) J^{-1}(\eta) \\
g_{\eta}(\eta) & =J^{-T}(\eta) g(\eta) \\
\tau_{\eta}(\eta) & =J^{-T}(\eta) \tau(\eta)
\end{aligned}
$$

(2.26)-(2.28) are used extensively in the implementation of the 6 DOF sliding controller.

\subsubsection{Notes on the Matrices in the Equations of Motion}

Note that $M$ and $D(v)$ are positive definite since they are the sum of positive definite matrices and that $C(v)$ is skew-symmetric since it the sum of two skew-symmetric matrices. From (2.28), it is straightforward to demonstrate that for nonsingular $J(\eta)$, $M_{\eta}(\eta)$ and $D_{\eta}(v, \eta)$ are also positive definite, though $C_{\eta}(v, \eta)$ is not skew-symmetric in general (see Fossen [1994]).

Another useful matrix relationship in the development of the 6 DOF sliding controller is:

$$
\dot{M}-2 C(v)
$$


Assuming that $M$ is equal to zero - i.e. the added mass terms in $M$ are independent of wave frequency and thus vehicle speed - then it is apparent that (2.29) is a skewsymmetric matrix ${ }^{1}$. Applying the transformations of (2.28) to (2.29) reveals that:

$$
\dot{M}_{\eta}-2 C_{\eta}(v, \eta)
$$

is also skew-symmetric. This last term, which is analogous to the matrix:

$$
\dot{H}(q)-2 C(q, \dot{q})
$$

in robotic manipulator control (see Slotine and Li [1991]) - which can be proved to be skew-symmetric and represents in matrix form conservation of energy in the manipulator ${ }^{2}$ - plays an important role in the 6 DOF sliding controller formulation.

\subsection{Hydrodynamic Coefficients}

Appendix I contains a summary of the body hydrodynamic coefficients identified in the equations of this chapter for the 21UUV of figure 1.1. The coefficients are the result of a combination of: 1) slender-body (strip) theory calculations; 2) aerodynamic lift and drag calculations; 3) comparison with a similar UUV upon which extensive computer modeling was performed (see Humpheys [1989]); and 4) tow-tank experiments made on a scale-model of the 21UUV (see Willy [1994] for a more complete accounting

\footnotetext{
${ }^{1}$ Actually the added mass terms in $M$ vary, albeit slightly, with varying sea conditions. This relationship is not known in general for each sea condition in $\mathfrak{R}^{6}$.

${ }^{2} \dot{H}(q)$ is the manipulator inertia matrix, $C(q, \dot{q})$ is the centripetal and Coriolis matrix and $q$ is the vector of joint angles. The fact that (2.31) is skew-symmetric lends some credence to the assumption that (2.29) and (2.30) are skew-symmetric .
} 
of these body and hydrodynamic coefficients).

As is well known, many of these coefficients vary significantly with Reynolds number and thus with vehicle speed. In addition, imperfect knowledge of the exact effect various vehicle components such as fins and propulsor shrouds have on these coefficients over the range of vehicle speeds is had. Thus, a great deal of uncertainty exists in the stated values of body and hydrodynamic coefficients - a large reason why robust control techniques such as sliding control are popular with underwater vehicles. As indicated in Appendix I, 5\% uncertainty in the body coefficients and a $20 \%$ uncertainty in the hydrodynamic coefficients were assumed. These uncertainties were used to differentiate the "true" vehicle model from the "estimated" vehicle model for simulation purposes in addition to their use in the actual design of the sliding controller. 


\section{Chapter 3}

\section{DOF Sliding Controller}

\subsection{General Discussion}

The use of nonlinear sliding control design to maintain stability and to ensure consistent performance despite the significant parameter and modeling uncertainties that characterize UUV design has been effectively employed for a number of years (e.g. Yoerger et al [1986], Dougherty and Woolweaver [1990], Hills and Yoerger [1994]). As demonstrated in the previous chapter, these vehicle equations of motion are highly coupled and nonlinear and consequently are often subjected to various simplifying assumptions to meet various design objectives (e.g. decoupled speed control, inner pitch/outer depth loop control, etc.). Such assumptions reduce the number of control inputs that have to be considered for the simplified problem at hand to one, allowing scalar sliding surface controller theory to be used. This theory is briefly summarized in section 3.2 to highlight some of the more important points of sliding control.

More demanding underwater vehicle maneuvers such as hovering or operation in

the dynamically difficult regime of shallow water, however, require careful consideration 
of the coupling between the various control inputs inherent in the equations of motion. Scalar sliding control becomes less satisfactory for such complex demands on the vehicle and, instead, a complete 6 DOF sliding controller (i.e. a 6 element vector sliding surface) should be considered which can handle the coupling between the various controls. Such a controller is developed in section 3.3 and Appendix II.

The resultant 6 DOF controller is then tested by computer simulation as summarized in section 3.4 with the very restrictive assumption that the 6 DOF commanded control vector produced by the sliding controller can be generated by the vehicle. This assumption is relaxed in chapter 4 .

\subsection{Scalar Sliding Control}

Consider a single-input dynamic system of the form:

$$
x^{(n)}=f(x)+b(x) u
$$

where scalar $x$ is the output, $x$ is the state vector, and scalar $u$ is the input. Defining the tracking error vector to some desired trajectory vector $\boldsymbol{x}_{d}$ as:

$$
\tilde{x}=x-x_{d}
$$

and defining a time-varying surface $S(\mathrm{t})$ in the state space by the scalar equation $s(x ; \mathrm{t})=0$ where:

$$
s(x ; t)=\left(\frac{d}{d t}+\lambda\right)^{n-1} \tilde{x} ; \lambda>0
$$

then (assuming the initial tracking error is zero) the $\mathrm{n}$-th order tracking problem of (3.1) 
and (3.2) is equivalent to remaining on the surface $S(t)$ for all $t>0$ - i.e. have effectively reduced the $\mathrm{n}$-th order tracking problem to a first-order stabilization problem in the scalar $s$. By choosing the input $u$ so that outside $S(\mathrm{t})$ :

$$
\frac{1}{2} \frac{d}{d t} s^{2} \leq-\eta|s| ; \eta>0
$$

it can easily be demonstrated (see Slotine and Li [1991], for example) that the surface $S(\mathrm{t})$

will be reached in a finite time less than $s|(t=0)| / \eta$; whereupon the tracking error will decrease exponentially to zero. (3.4) is referred to as the sliding condition and it is possible to choose the control input $u$ such that (3.4) is met even in the presence of parameter uncertainties or unstructured uncertainties in the dynamics. This is why sliding control is so useful for UUV controller design; not only can it handle non-linearities, but a degree of robustness can be factored into the controller.

\subsection{Vector Sliding Control}

To capture the full power of sliding control theory for an UUV intended to operate in shallow water or designed to perform complex maneuvers such as hovering, it is necessary to expand scalar sliding control to vector sliding control. Fortunately, as has been alluded to, the particular structure of (2.26) and (2.27) make the development of the 6 DOF sliding controller a relatively straightforward task. The derivation that follows is analogous to the multi-input, multi-output (MIMO) robust robot trajectory controller outlined in Slotine and Li [1991] and is similar to the adaptive MIMO sliding controller discussed in Fossen [1994]. 
In the $6 \mathrm{DOF}$ sliding controller, the tracking dynamic variable $s$ of the SISO sliding controller is replaced by a 6-element vector $\boldsymbol{s}$ which is defined as:

$$
S=\dot{\tilde{\eta}}+\Lambda \tilde{\eta}=\dot{\eta}-\dot{\eta}_{r}
$$

where:

$$
\tilde{\eta}=\eta-\eta_{d} ; \dot{\eta}_{r}=\dot{\eta}_{d}-\Lambda \tilde{\eta}
$$

and where:

$$
\begin{aligned}
\boldsymbol{\Lambda} & =\text { control bandwidth matrix }\left(\boldsymbol{\Lambda}=\boldsymbol{\Lambda}^{T}>0\right) \\
\boldsymbol{\eta}_{d} & =\text { desired earth-fixed trajectory } \\
\boldsymbol{\eta}_{r} & =\text { reference earth-fixed trajectory }
\end{aligned}
$$

The control bandwidth matrix is discussed in section 3.4. The reference (or virtual) trajectory is merely a notational manipulation which transforms the energy-related properties of the vehicle (in terms of $\eta$ ) into trajectory control properties (in terms of $\boldsymbol{s}$ ). Defining the Lyapunov function:

$$
V(t)=1 / 2 s^{T} M_{\eta} s>0
$$

Differentiating (3.8) and substituting the derivative of (3.5) yields:

$$
\dot{V}(t)==s^{T}\left(M_{\eta} \ddot{\eta}-M_{\eta} \ddot{\eta}_{r}\right)+1 / 2 s{ }^{T} \dot{M}_{\eta} s
$$

Noting that the system dynamics (2.27) can be rewritten by means of (3.5) as:

$$
M_{\eta} \ddot{\eta}=\tau_{\eta}-\left(C_{\eta}+D_{\eta}\right)\left(s+\dot{\eta}_{r}\right)-g_{\eta}
$$

Then (3.9) can be recast as: 


$$
\dot{V}(t)=s^{T}\left(\tau_{\eta}-M_{\eta} \ddot{\eta}_{r}-\left[C_{\eta}+D_{\eta}\right] \dot{\eta}_{r}-g_{\eta}\right)-s^{T} D_{\eta} s
$$

Where the fact that the quantity:

$$
\dot{M}_{\eta}-2 C_{\eta} \text { is skew symmetric } \forall \eta
$$

is used to eliminate the expression:

$$
s^{T}\left(\dot{M}_{\eta}-2 C_{\eta}\right) s
$$

See Fossen [1994] and Slotine and Li [1991] - the latter in the context of robot manipulators - for explanation/"proof"; this term expresses the matrix form of the conservation of energy in the system. The skew-symmetry of (3.12) has been discussed previously in Chapter 2.

Then, choosing the control law as:

$$
\tau_{\eta}=\hat{\tau}_{\eta}-k(\eta) \operatorname{sgn}(s)
$$

With:

$$
\hat{\tau}_{\eta}=\hat{M}_{\eta} \ddot{\eta}_{r}+\left(\hat{C}_{\eta}+\hat{D}_{\eta}\right) \dot{\eta}_{r}+\hat{g}
$$

Where $\mathbf{k}(\eta) \operatorname{sgn}(\mathbf{s})$ is defined as the vector of components $\mathrm{k}_{i}(\eta) \operatorname{sgn}\left(\mathrm{s}_{i}\right)$ and where the estimated (hat) system matrices are linearly related to the actual plant matrices by error (tilde) matrices, for example:

$$
\tilde{M}_{\eta}=\hat{M}_{\eta}-M_{\eta}
$$

Thus (3.14) is the control input which would make (3.11) equal to zero if the vehicle dynamics were exactly known. Then the Lyapunov function time derivative becomes 
with the use of (3.14):

$$
\dot{V}(t)=s^{T}\left[\tilde{M}_{\eta} \ddot{\eta}_{r}+\left(\tilde{C}_{\eta}+\tilde{D}_{\eta}\right) \dot{\eta}_{r}+\tilde{g}_{\eta}\right]-\sum_{i=1}^{6} k_{i}(\boldsymbol{\eta})\left|s_{i}\right|-s^{T} D_{\eta} s
$$

Choosing $k_{i}(\eta)$ such that:

$$
k_{i}(\eta) \geq\left|\left[\tilde{M}_{\eta} \ddot{\eta}_{r}+\left(\tilde{C}_{\eta}+\tilde{D}_{\eta}\right) \dot{\eta}_{r}+\tilde{g}_{\eta}\right]_{i}\right|+\epsilon_{i} ; \epsilon_{i}>0
$$

Ensures:

$$
\dot{V}(t) \leq\left(-\sum_{i=1}^{6} \epsilon_{i}\left|s_{i}\right|-s^{T} D_{\eta} s\right) \leq 0
$$

Thus this 6 DOF sliding controller is asymptotically stable from anywhere in the state space (except for at pitch angles of \pm 90 degrees) as demonstrated in Appendix II. Note the dissipative nature of the drag matrix actually causes the system to reach the desired trajectory faster than if it did not exist.

As formulated above, the $6 \mathrm{DOF}$ sliding controller is a switching or chattering controller; resulting in very high control action and probable excitation of unmodelled vehicle dynamics. As indicated in Slotine and Lee [1991], this potential problem can be avoided by the modification of the control law (3.14) to include a smoothing term near the switching surface characterized by a boundary layer thickness $\phi_{i}$. The control law becomes:

$$
\tau_{i_{\eta}}=\hat{\tau}_{i_{\eta}}-k_{i}(\eta) \operatorname{sat}\left(\frac{s_{i}}{\phi_{i}}\right)
$$

The inclusion of this boundary layer effectively trades-off some tracking precision for some robustness to unmodelled dynamics. By making the boundary layer time-varying: 


$$
\tau_{\eta}=\hat{\tau}_{\eta}-\bar{k}_{i}(\eta) \operatorname{sat}\left(\frac{s_{i}}{\phi_{i}}\right)
$$

where:

$$
\bar{k}_{i}(\eta)=k_{i}(\eta)-\dot{\phi}_{i}
$$

and:

$$
\dot{\phi}_{i}=k_{i}\left(\eta_{d}\right)-\lambda \phi_{i}
$$

some of the tracking precision can be regained. See Slotine and Li [1991] for a more complete discussion on smoothing out the control discontinuity of (3.14). All simulations in this study utilize the smooth (sat) vector sliding controller with the values for the boundary layer thickness $\phi_{i}^{\prime}$ s chosen as .1 for all DOF's (this value seemed to yield good control signals in terms of smoothness).

\subsection{Simulations}

In order to implement the 6 DOF sliding controller that was designed in the previous section, it must be noted that the controller was developed in the earth-fixed frame of reference. In one sense, this is desirable as the desired trajectories (e.g. executing a trackline change) are usually earth-fixed in nature. However, the state measurements are made and the commanded control actions are carried out in the vehicle reference frame. Thus, the controller implementation logic should go something like: get 
the vehicle-fixed velocities/accelerations in all $6 \mathrm{DOF}^{1}$; compute the body-referenced matrices of (2.26); convert the body-reference matrices to earth-referenced matrices by use of (2.28); get desired earth-referenced control vector from the sliding controller; then compute the desired vehicle-referenced control vector using (2.28). In this section, the assumption is made that the desired vehicle-referenced control vector is instantly produced in all 6 DOF's by the vehicle control system - more realistic scenarios are examined in the next chapter.

A comment on the bandwidth matrix $\Lambda$. As discussed in Slotine and Li [1991], the particular elements in the matrix should be chosen as high as possible (to achieve performance improvement) without exciting any unmodelled dynamics such as resonant modes or to interfere with any unmodelled time-delays (e.g. actuator response). With this

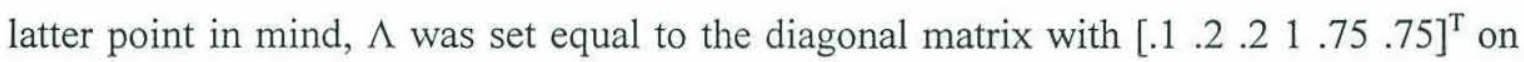
the main diagonal. These values are representative of the possible rate-of-response in the various DOF's.

A functional, 6 DOF sliding controller based on the preceding discussion was implemented using MATLAB using the 21UUV body and hydrodynamic coefficients of Appendix I. Figure 3.1 depicts the desired and actual depth and trackline responses in a coordinated 5 meter rise / 5 meter shift in trackline occurring over a 40 second period of time with the vehicle attempting to maintain a constant speed of 5 knots. A slight $(+10 \mathrm{~N}$ more buoyancy) mismatch in weight/buoyancy was assumed, as were slightly non-

${ }^{1}$ The 21UUV of figure 1.1 actually has excellent state measurement capabilities (including accelerations). No state observers are necessary. 
zero values for $\mathrm{x}_{\mathrm{G}}$ and $\mathrm{z}_{\mathrm{G}}$. 
Commanded Rise \& Heading Change w/ "Perfect" 6DOF Control
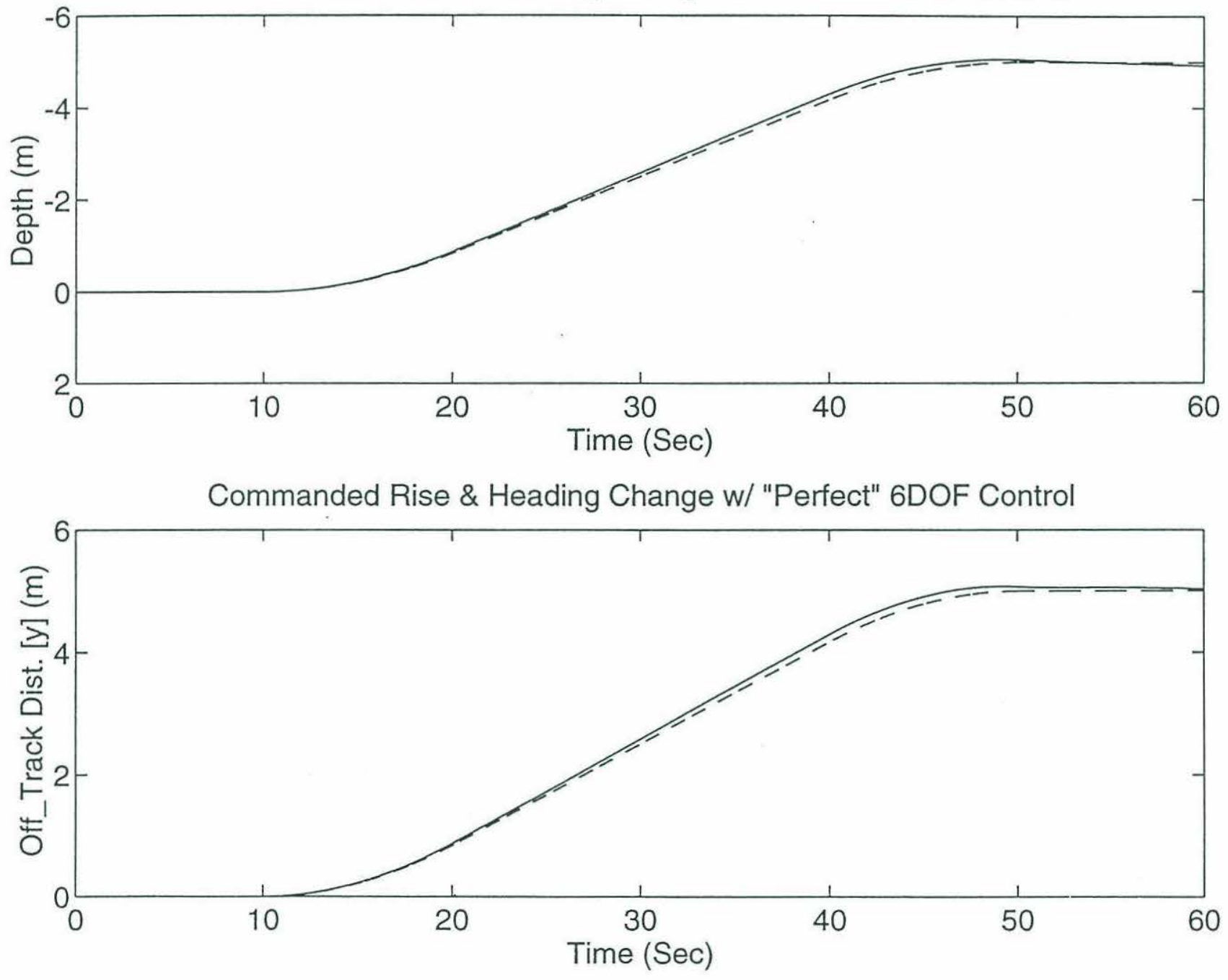

Figure 3.1 - Comparison of Actual vs. Desired Vehicle Response During a Coordinated Maneuver Assuming Full 6 DOF Control Authority Available. The vehicle follows the commanded trajectories (dotted lines $-5 \mathrm{~m}$ rise coupled with a $5 \mathrm{~m}$ trackline change) extremely well as is expected assuming the availability of full 6 DOF control authority as might be the case for a vehicle designed to hover. The error between the actual and desired response is due to the assumed errors in the body and hydrodynamic coefficients. Also this simulation assumed a positive $10 \mathrm{~N}$ buoyancy. 


\section{Chapter 4}

\section{DOF Implementation of the 6 DOF Sliding Controller}

\subsection{General Discussion}

The 6 DOF control authority assumed to exist in the simulation of figure 3.1 rarely exists in practical UUV's - except for a very few specialized vehicles which are specifically designed for demanding maneuvers such as hovering. Rather, the typical UUV will have a limited number of controllable DOF's such as the four available to the 21UUV of figure 1.1 - sternplanes, rudder, ailerons and propulsor (thrust). This chapter will present the development of a working 4 DOF implementation of the 6 DOF sliding controller for the 21UUV which will be shown to be effective for a broad range of useful commanded maneuvers. This 4 DOF implementation relies on the well-known nonminimum phase relationship between the pitch/depth and yaw/trackline DOF's and is similar in concept to the inner/outer loop pitch(yaw)/depth(heading) controllers currently used in scalar sliding or PID controllers for UUV's that are assumed to be decoupled in the pitch-yaw planes. An argument - not proof - will be presented as to why this 
controller implementation works; an argument supported by a number of computer simulations. This argument also suggests directions in which further study might be pursued to improve this 4 DOF controller implementation and how to develop other controllers based on UUV's with different combinations of available control surfaces/thrusters than the 21UUV of figure 1.1 .

\subsection{DOF Commanded to 4 DOF Available Control}

The fundamental problem is the conversion the commanded $\tau$ vector - the bodyreferenced force/moment vector converted from the earth-referenced $\tau_{\eta}$ output of the 6 DOF sliding controller (3.28) - to proper control signals for the UUV thrusters/control surfaces.

For example, the 21UUV of figure 1.1 has four controllable DOF - two sternplanes $\left(\delta_{s}\right)$ - which directly influence the pitch/depth DOF's; two rudders $\left(\delta_{r}\right)$ - which directly influence the yaw/trackline DOF's; a thruster (propulsor) (T) - which directly controls the forward speed and thus the longitudinal motion; and ailerons $\left(\delta_{a}\right)$ - which directly control the roll angle. The aileron's action is accomplished by appropriate opposing settings on the two sternplanes and/or rudders. There is also some coupling between the various controls - e.g. the rudders affect roll to a degree.

Note that the sternplanes and rudders must each control two degrees of freedom in the 21UUV configuration. In scalar sliding control design (e.g. Fossen [1994], Hills and Yoerger [1994]), this is typically managed by using inner pitch/outer depth loops (similarly, inner yaw/outer trackline loops) in which the desired pitch angle at each time 
step is generated by the depth trajectory error at that time step. To maintain the matrix/vector format of 6 DOF sliding controller, the vector sliding control design considered in this study employs a least-squares methodology to overcome this problem as detailed in the following sub-sections.

\subsubsection{Unweighted Least Squares}

The control inputs for the 21 UUV can be grouped as a vector $\boldsymbol{u}_{\text {body }}$ which is related to $\tau$ by:

$$
\tau=B u_{b o d y}=B\left[T \delta_{s} \delta_{r} \delta_{a}\right]^{T}
$$

As stated, the problem is that the $B$ matrix relating the commanded force/moment vector to the available force moment vector is $6 \times 4$; that is, (4.1) is an underdetermined system of equations for which, in general, no exact solution exists. However, the error $e$ between $\tau$ and $B u_{b o d y}$ where:

$$
e=\tau-B u_{b o d y}
$$

can be minimized by minimizing the length of $\left.|| e\right|^{2}=\left(\tau-B u_{b o d y}\right)^{\mathrm{T}}\left(\tau-\boldsymbol{B} \boldsymbol{u}_{\text {body }}\right)$; the least squares solution. From standard matrix theory (e.g. Strang [1986]), the unweighted least squares solution to (4.1) is:

$$
\hat{u}_{b o d y}=\left(B^{T} B\right)^{-1} B^{T} \tau
$$




\subsubsection{Weighted Least Squares}

Unfortunately, straightforward application of (4.3) leads to unstable control action for any $21 U U V$ typical maneuver. This can be seen from the coefficients that govern the pitch/depth and yaw/heading. For instance, consider the hydrodynamic coefficients relating rudder angle to yaw and heading $-N_{\delta r}$ and $Y_{\delta r}$. From Appendix I, the coefficients are opposite in sign, meaning that for a course change in which both a yaw angle and heading trajectory in the same directions would be commanded, (4.3) would be trying to the impossible feat of moving the rudder in opposite directions at the same time! The same analysis holds for the pitch/depth coupled maneuvers (taking into account that $+\mathrm{z}$ is defined in the down direction).

This is a well-known problem of not only torpedo-shape underwater vehicles such as the 21UUV, but also of aircraft (see Slotine and Li [1991], for example). The solution to maneuvering vehicles that possess such characteristics is to first operate the control surface to get the desired angle-of-attack, then to reverse the direction of the control surface so that the translational portion of the maneuver (depth or trackline) is satisfied. Such behavior is termed non-minimum phase, and an appropriate way must be found to incorporate such behavior into the mathematics of (4.3).

One way to accomplish this would be to command proper desired trajectories to follow so that the correct non-minimum phase response would be enacted by the vehicle. Unfortunately, this is an open-loop solution and is of doubtful utility when parameter and modelling uncertainties are introduced, not to mention any disturbances the vehicle would experience in shallow-water. 
A better solution is to modify (4.3) so that it captures the non-minimum phase characteristics of the 21UUV - that is, the vehicle angle orientation is achieved first (faster time constant), then the slower lateral or vertical translation takes place. Looking at the $\Lambda$ matrix of the $6 \mathrm{DOF}$ sliding controller (3.18), we see this has been partially accomplished by the performance weightings on the DOF's; the .75 value for pitch and yaw allows faster vehicle orientation response that the .2 value for depth and heading. Then, weighting the error $e$ by a matrix $W$ such that:

$$
\boldsymbol{W e}=\left[\begin{array}{cccccc}
\lambda_{X} & 0 & 0 & 0 & 0 & 0 \\
0 & \lambda_{Y} & 0 & 0 & 0 & 0 \\
0 & 0 & \lambda_{Z} & 0 & 0 & 0 \\
0 & 0 & 0 & \lambda_{K} & 0 & 0 \\
0 & 0 & 0 & 0 & \lambda_{M} & 0 \\
0 & 0 & 0 & 0 & 0 & \lambda_{N}
\end{array}\right] \boldsymbol{e}
$$

where the weights $\lambda_{i}$ and the desired trajectories are properly chosen forces the vehicle into the correct non-minimum phase behavior to achieve commanded trajectories as is argued in the following sub-section. ${ }^{1}$ Defining $C=W^{\mathrm{T}} W$, then (4.3) becomes in weighted least squares (e.g. Strang [1986]):

$$
\hat{u}_{b o d y}=\left(B^{T} C B\right)^{-1} B^{T} C \tau
$$

After some trial and error as documented in the following simulations of the

${ }^{1}$ Considering the significant coupling between pitch and depth DOF's for example it might be prudent to actually consider a non-diagonal form for $W$ - this was not examined in this study. 
following section, a "good" $W$ was determined to be:

$$
W=\left[\begin{array}{cccccc}
1 & 0 & 0 & 0 & 0 & 0 \\
0 & 1 & 0 & 0 & 0 & 0 \\
0 & 0 & 1 & 0 & 0 & 0 \\
0 & 0 & 0 & 1 & 0 & 0 \\
0 & 0 & 0 & 0 & .06 & 0 \\
0 & 0 & 0 & 0 & 0 & .06
\end{array}\right]
$$

\subsubsection{Justification for the Weighted Least Squares Method}

For simplicity and clarity, only a 21UUV dive is considered; by vehicle symmetry similar arguments apply to vehicle climbs and trackline changes.

A key assumption in this methodology is that the desired vehicle pitch angle, angular rate and acceleration are always chosen to be zero - only the z-axis has non-zero desired $\eta_{d}, \dot{\eta}_{d}$ and $\ddot{\eta}_{d}$ during the dive (along with x-axis non-zero $\eta_{d}$ and $\dot{\eta}_{d}$ ). Then, from (3.6), initial non-zero reference trajectories $\eta_{r}$ and $\dot{\eta}_{r}$ in the $x$ - and z-axes are generated, which in turn from the sliding control law (3.14) generates earth-reference commands $\tau_{\eta}$ in these two DOF's. ${ }^{2}$

The non-zero reference trajectories also generate an initial non-zero $\tau_{\eta}$ in the pitch DOF due to coupling coefficients between the pitch and $x / z$ DOF's that exist in the matrices of (3.14). This commanded moment can be viewed as a feed-forward term that

\footnotetext{
${ }^{2}$ Note that the distinction clear between the body- and earth-referenced frames is not rigorously maintained in this particular analysis. Considering the $J$ matrix would only obscure the relevant ideas; anyway $J$ is very close to being the identity matrix throughout the analysis.
} 
is balancing/negating the moment that is generated when diving. A study of these coupling coefficients reveals that this commanded moment requires the sternplanes to move in the opposite direction that the commanded force for the dive requires. ${ }^{3}$

Thus to satisfy this non-minimum phase characteristic between pitch and dive, the pitch demand must be satisfied first as previously discussed. This is accomplished by underweighting pitch relative to depth in the weighting matrix $W$. To understand how this works, consider only the depth and pitch axes for a moment which results in the unweighted least squares solution of (4.3) to look like:

$$
\delta_{s}=(\text { forw. vel. })^{-2}\left[\frac{\tau_{z \text {-dir }}}{Z_{\delta_{s}}}+\frac{\tau_{\text {pitch-dir }}}{M_{\delta_{s}}}\right]
$$

and the weighted least squares solution of (4.5) to look like:

$$
\delta_{s}=(\text { forw. vel. })^{-2}\left[\frac{\tau_{z-\text { dir }}}{Z_{\delta_{s}}}+\frac{\tau_{\text {pitch-dir }}}{(\text { pitchweight }) \times M_{\delta_{s}}}\right]
$$

See Appendix I for the values of the sternplane hydrodynamic coefficients. Comparing (4.7) and (4.8), it is apparent that increasing the underweighting the pitch weight forces the sternplanes to respond more quickly to the pitch command resulting from the control law of (3.14) and delays the implementation of the dive command. When optimally chosen, the pitch weighting results in the vehicle achieving the optimal pitch angle as it

\footnotetext{
${ }^{3}$ This assertion, though true for the particular magnitudes and signs of the relevant hydrodynamic coefficients listed in Appendix I, needs to be more fully analyzed for the all the possible variations in these magnitudes and signs to ensure that the weighting value in the $W$ matrix remains optimal and, more importantly, keeps the controller stable.
} 
executes the dive.

\subsection{Simulations}

Extensive numerical simulations are presented in this section to illustrate 21UUV response for a variety of commanded maneuvers and to further clarify the implications of the weighted least squares methodology utilized to obtain 6 DOF satisfactory vehicle control from 4 DOF control authority.

\subsubsection{Determination of Optimal $\lambda_{i}$ 's}

The control hydrodynamic coefficients used for the $B$ matrix of (4.1) are listed in Appendix I. The angle squared terms could not be directly included into this matrix else (4.5) could not be solved via normal matrix means. Fortunately, these terms are rather small for typical maneuvers and speeds and can safely be ignored. ${ }^{4}$

As is demonstrated from the figures there is an optimal weighting for the best depth-pitch response $-\lambda \approx .06$. As the next two sub-sections show, it appears this value is good over a range of speeds and dive rates.

${ }^{4}$ For the simulations that follow, the angle squared terms are included in the 21UUV's "real" dynamics for simulation purposes. As is evident from the simulation plots, vehicle response does not appear adversely affected. 

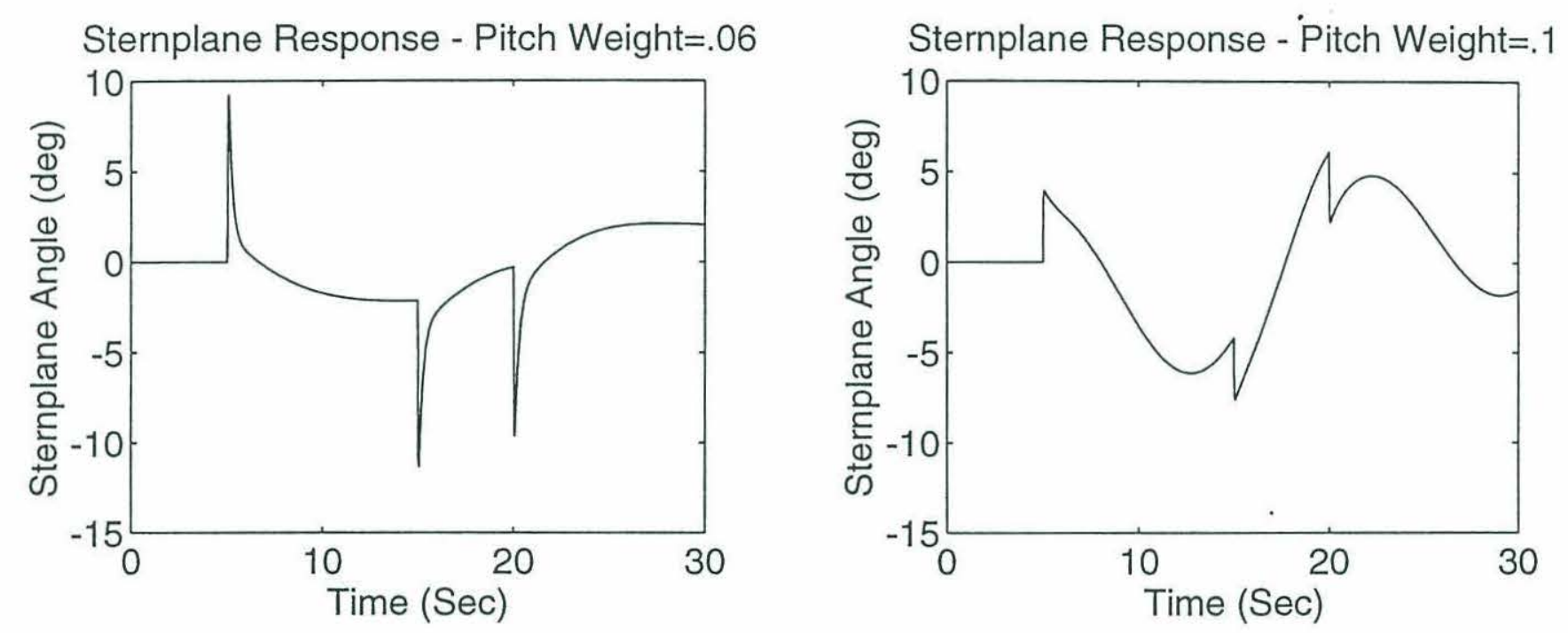

Figure 4.1 - Comparison of Sternplane Response in a Commanded Dive for Pitch Weights of $\lambda=.06$ and $\lambda=1$. Overweighting the pitch error weight relative to the depth error weight $(\lambda=.1 / 1$ vice $.06 / 1)$ overly restricts the range of allowed initial sternplane motion and results in the sternplanes being applied for too long at excessive angles during the remainder of the commanded maneuver with deleterious effects on vehicle dive response as shown in successive figures. Note the non-minimum phase action of the sternplanes - initially moving in one direction to get the requisite pitch angle for the commanded dive, then moving in the opposite direction to allow the vehicle to change depth. 
Depth Response - Pitch Weight $=.06$

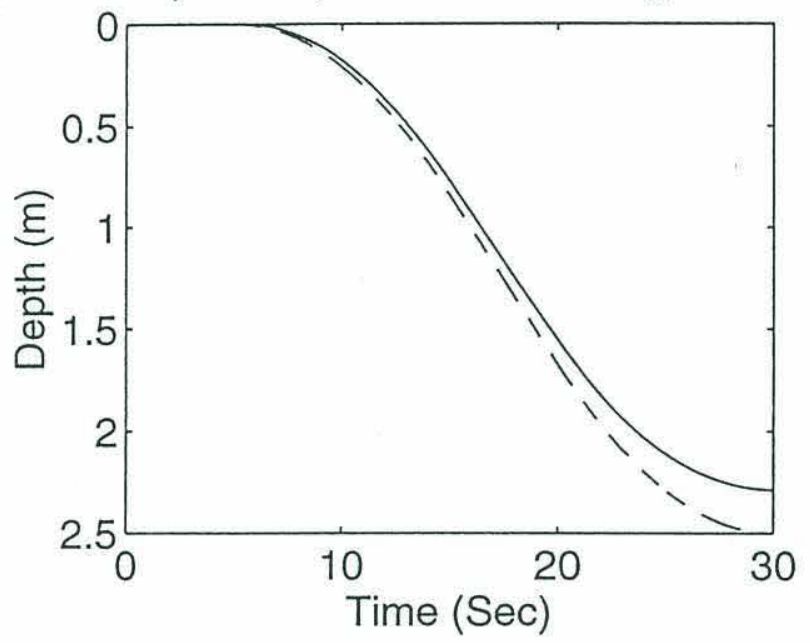

Depth Rate Response - Pitch Weight=.06

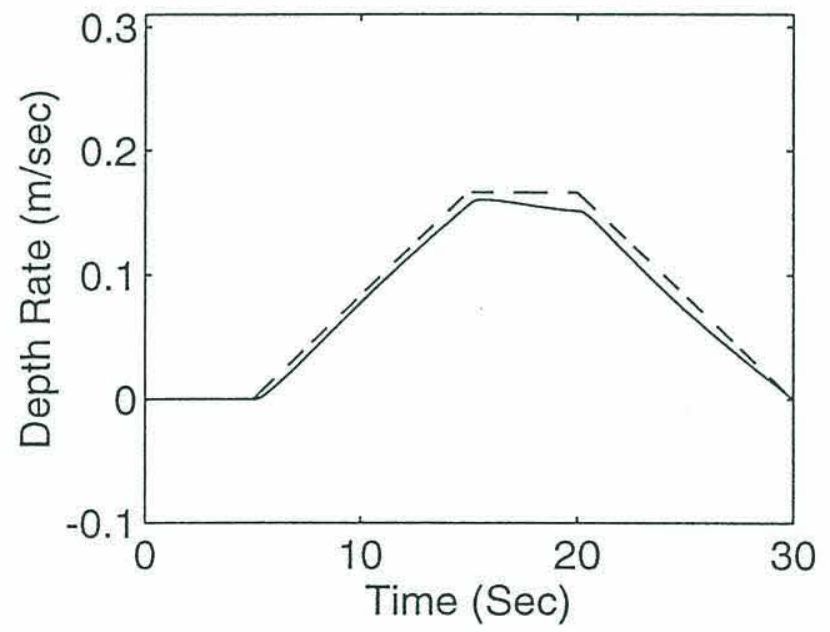

Depth Response - Pitch Weight=.1

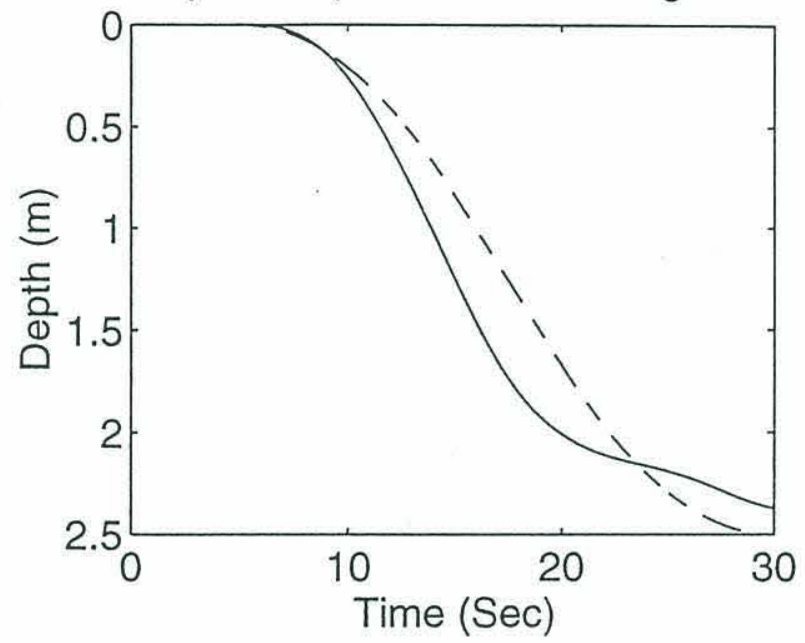

Depth Rate Response - Pitch Weight=.1

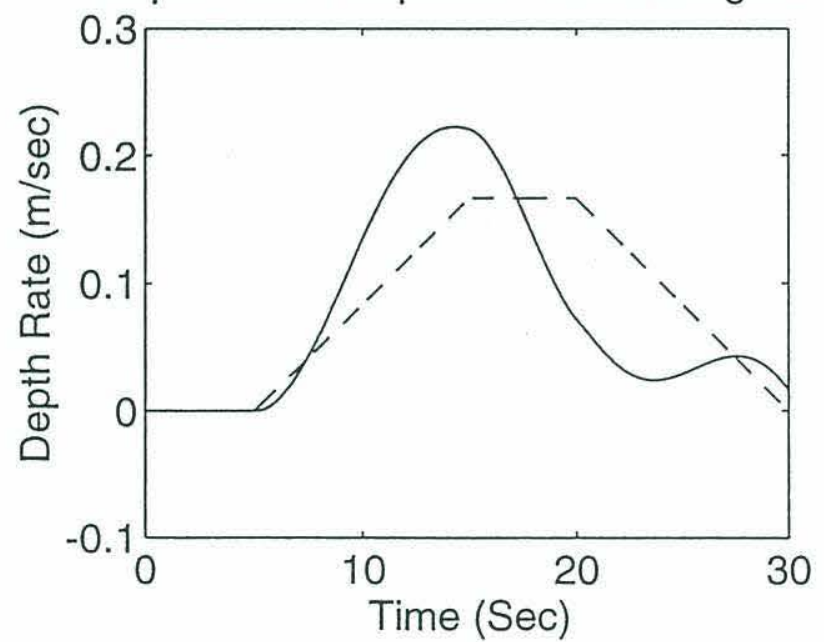

Figure 4.2 - Comparison of Depth and Depth Rate Responses in a Commanded Dive for Pitch Weights of $\lambda=.06$ and $\lambda=1$. Excessively high pitch weighting $(\lambda=.1$ vice .06$)$ results in the vehicle hunting about the commanded depth and depth rate trajectories (indicated by dotted lines). The vehicle's sternplanes do not initially achieve sufficient angles to get vehicle pitch to its optimal angle. The sliding controller - which is governed by the equations of motion and the commanded trajectories - then acts, in a simplified sense, as a lightly damped system as the sternplane angle and direction is dominated first by moment control, then depth control, etc. as it attempts to follow the depth change trajectory. 


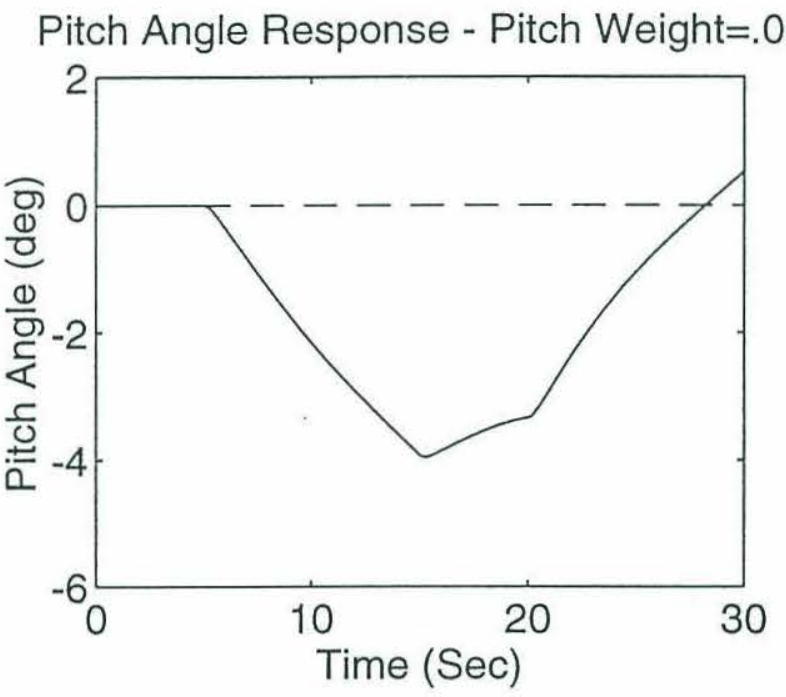

Pitch Rate Response - Pitch Weight $=.06$

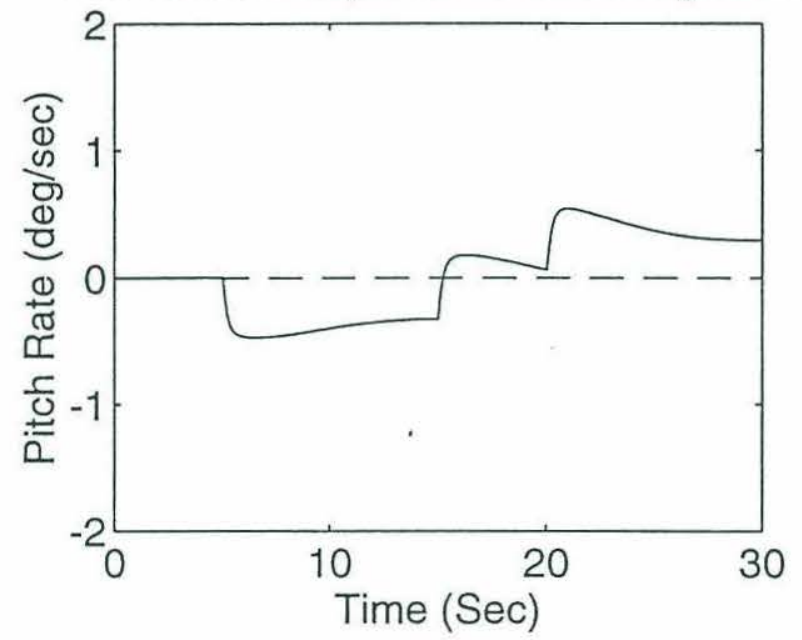

Pitch Angle Response - Pitch Weight=.1

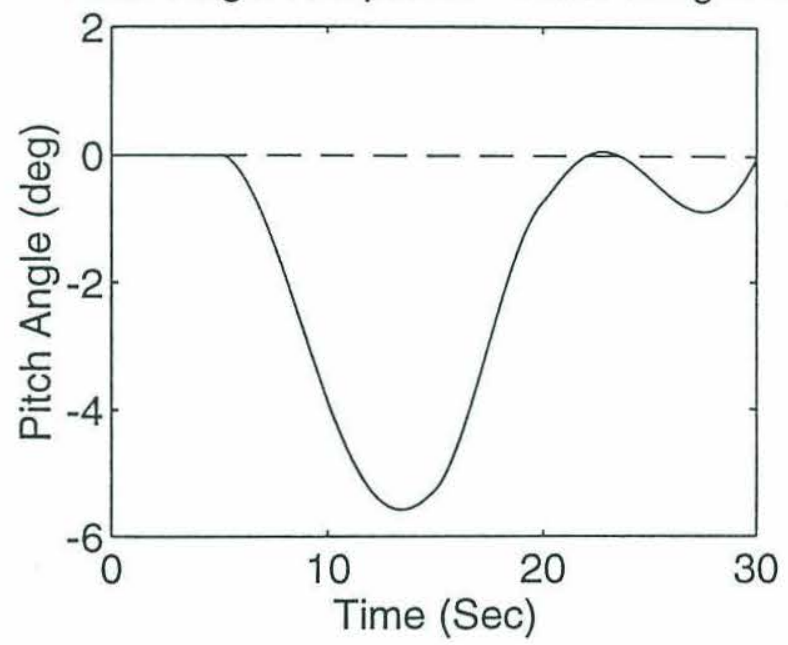

Pitch Rate Response - Pitch Weight=.1

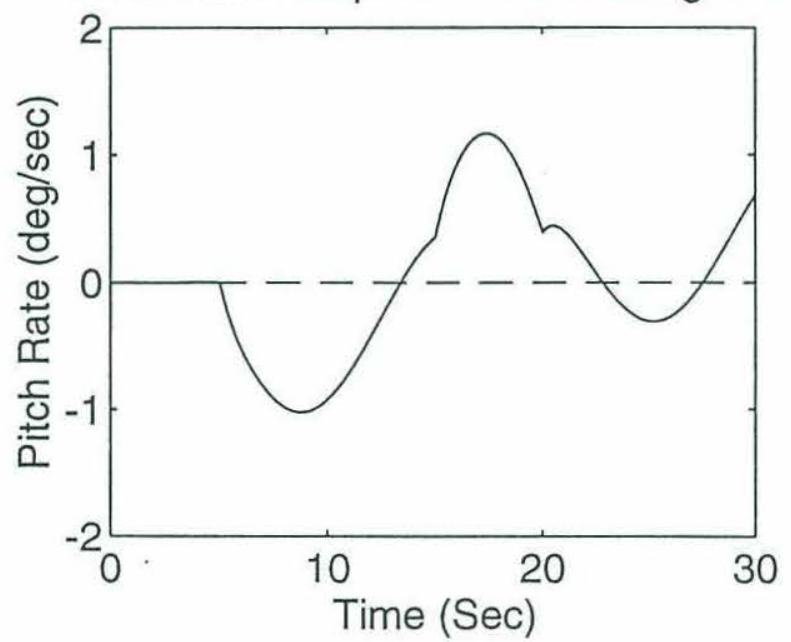

Figure 4.3 - Comparison of Pitch and Pitch Rate Responses in a Commanded Dive for Pitch Weights of $\lambda=.06$ and $\lambda=.1$. Limiting the allowed initial sternplane motion $(\lambda=.1$ vice .06$)$ results in the overall pitch response to be excessive. See figure 4.2 . 
Forward Speed - Pitch Weight=.06
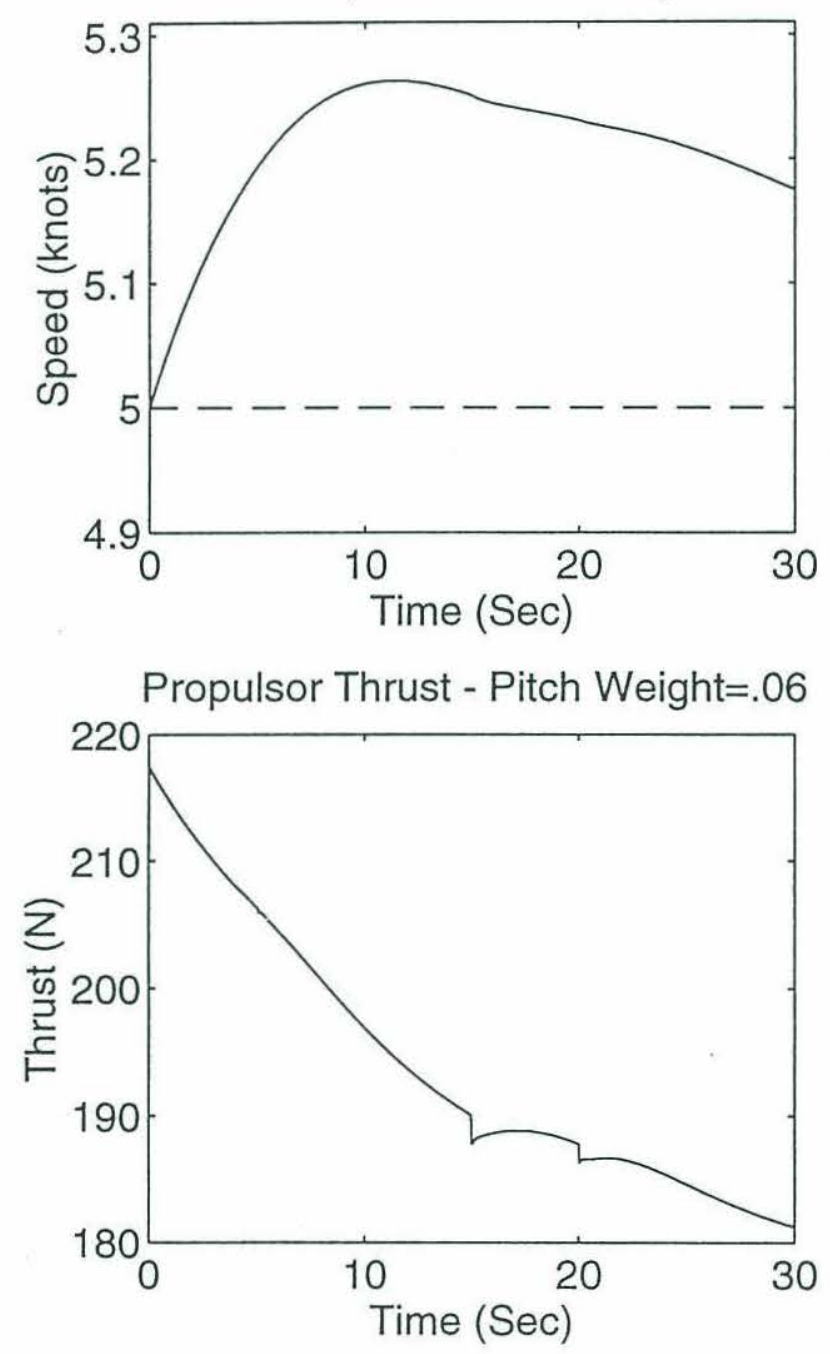

Forward Speed - Pitch Weight $=.1$
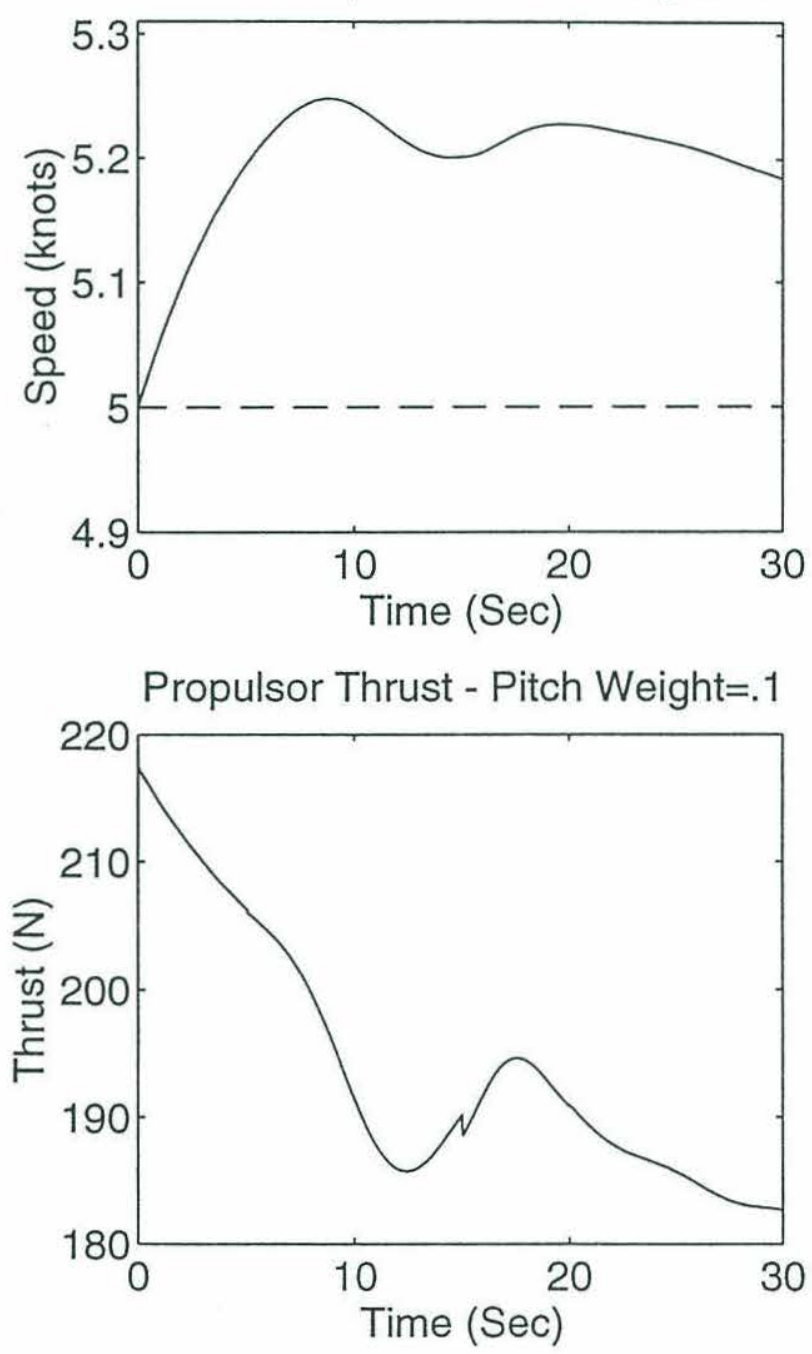

Figure 4.4 - Comparison of Forward Speed and Thrust Responses in a Commanded Dive for Pitch Weights of $\lambda=.06$ and $\lambda=1$. Similar responses for both pitch error weightings. Note that the initial overestimation of needed thrust is endemic to all the simulations of this study as a result of the particular assumed errors between the "true" and estimated values for the hydrodynamic coefficients. 

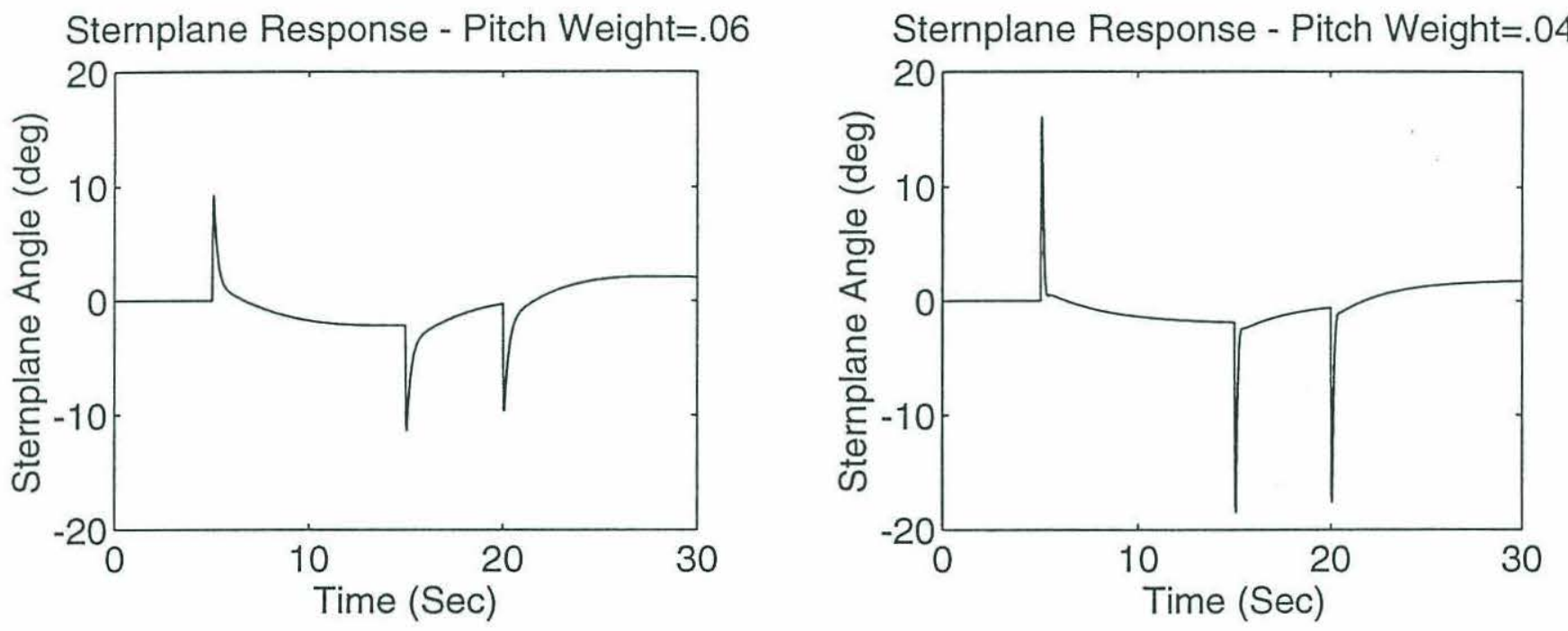

Figure 4.5 - Comparison of Sternplane Response in a Commanded Dive for Pitch Weights of $\lambda=.06$ and $\lambda=.04$. Underweighting the pitch error weight relative to the depth error weight $(\lambda=.04 / 1$ vice $.06 / 1)$ causes the initial sternplane motion to be overly excessive and results in the requisite pitch angle for optimal dive performance not being achieved as seen in the following figures. 

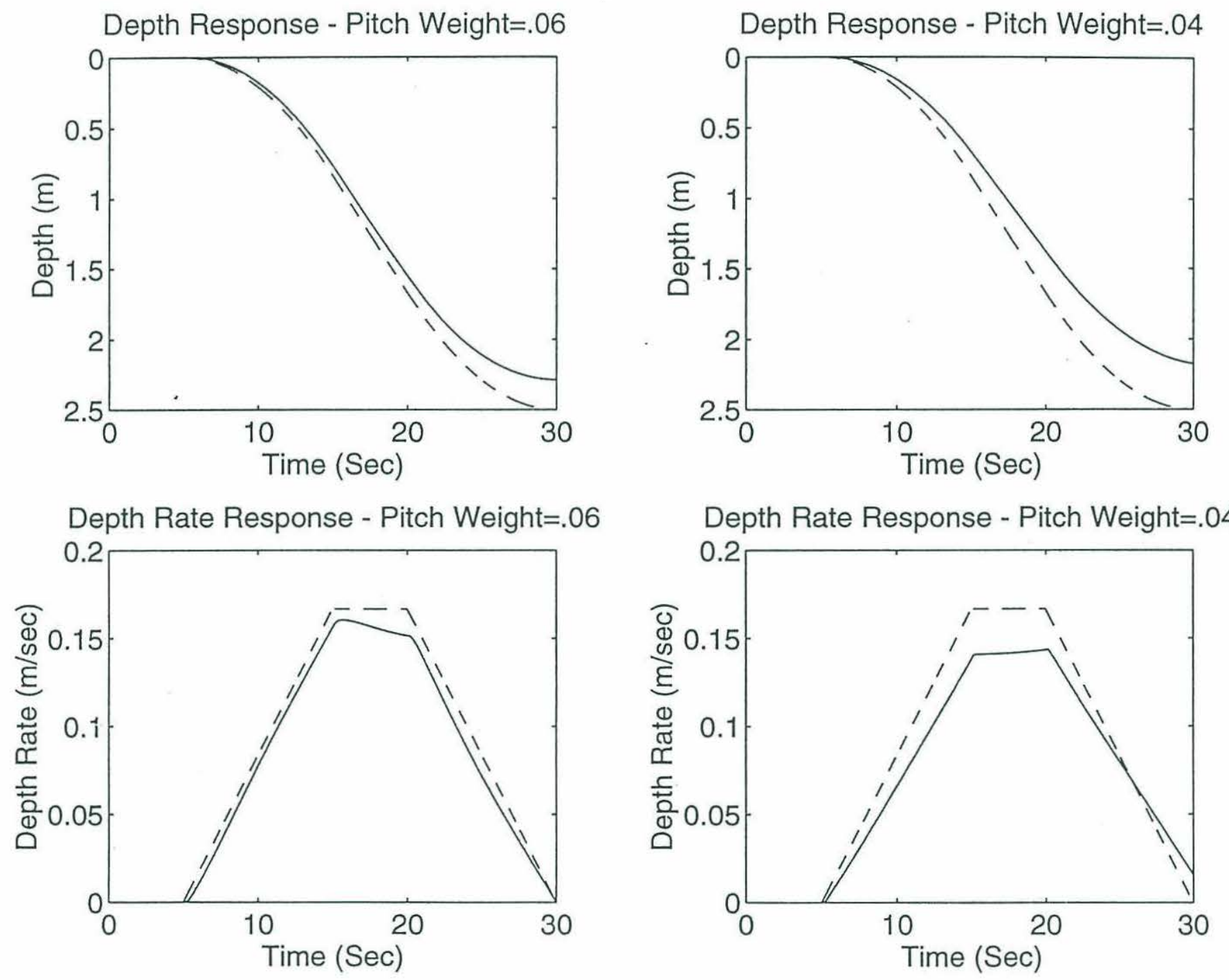

Figure 4.6 - Comparison of Depth and Depth Rate Responses in a Commanded Dive for Pitch Weights of $\lambda=.06$ and $\lambda=.04$. As evidenced in the next figure, the underweighting of the pitch weighting $(\lambda=.04$ vice .06) results in the vehicle not achieving the optimal pitch angle to best effect the commanded depth and depth rate trajectories (indicated by dotted lines). 


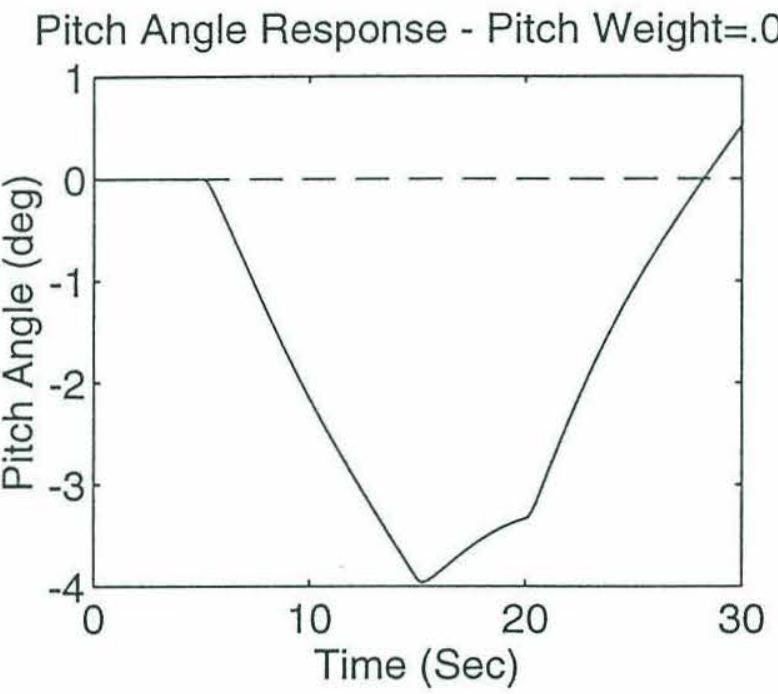

Pitch Rate Response - Pitch Weight=.06

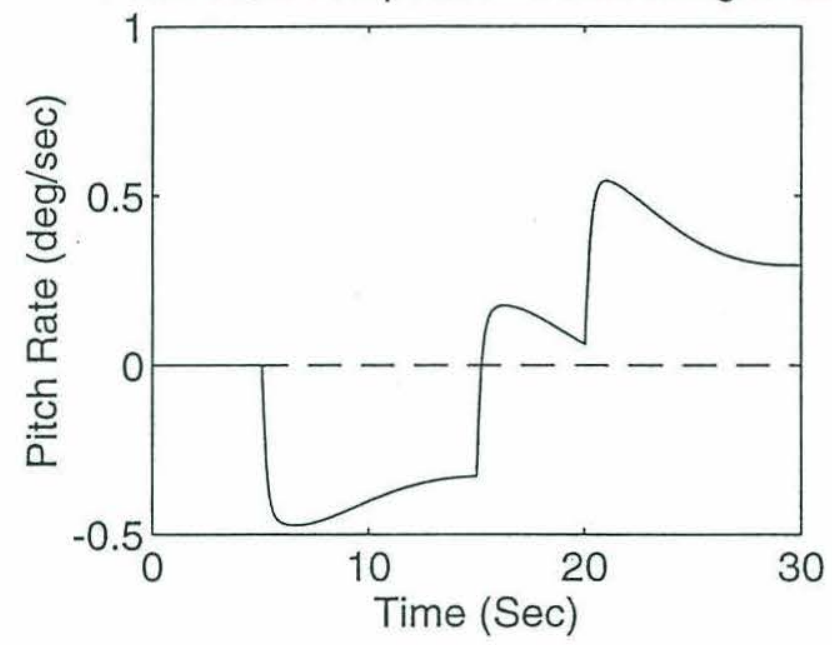

Pitch Angle Response - Pitch Weight $=.04$

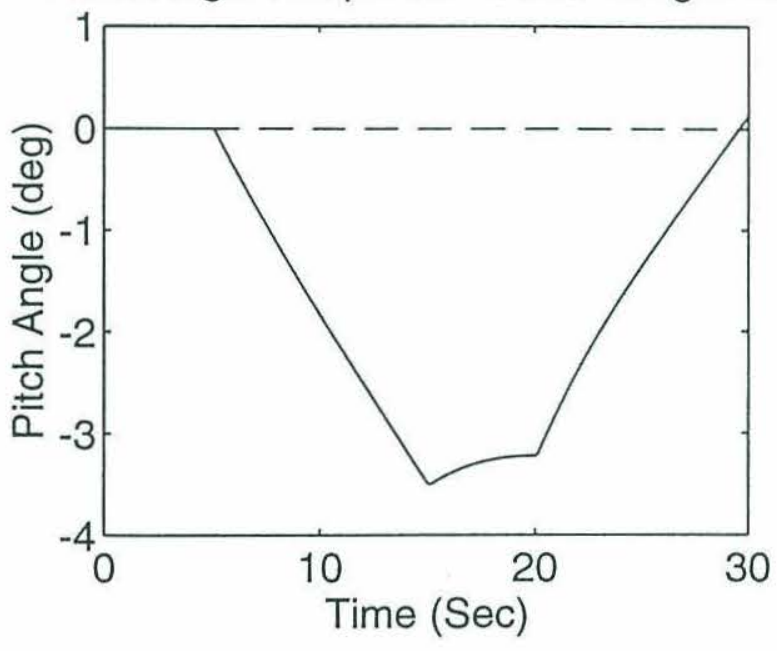

Pitch Rate Response - Pitch Weight=.04

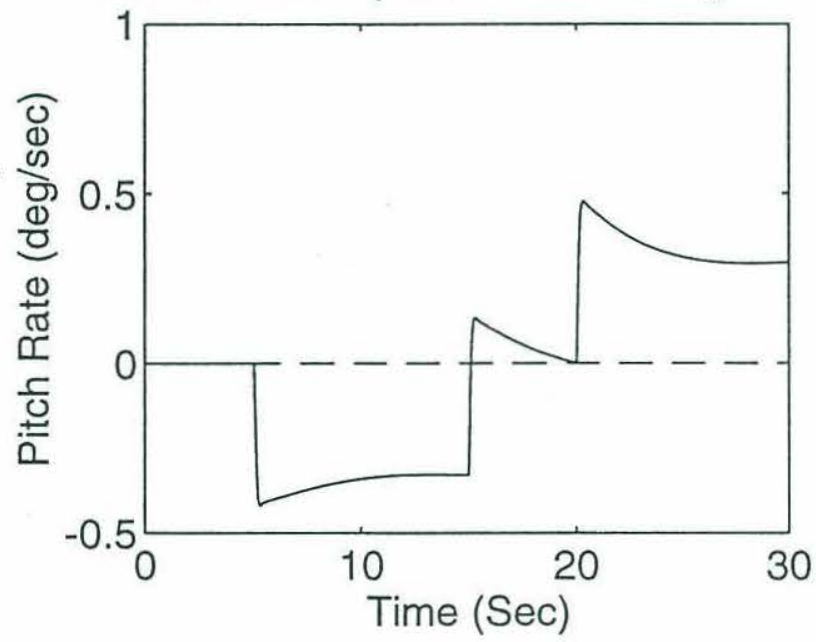

Figure 4.7 - Comparison of Pitch and Pitch Rate Responses in a Commanded Dive for Pitch Weights of $\lambda=.06$ and $\lambda=.04$. Underweighing the pitch error weighing ( $\lambda=.04$ vice .06$)$ actually results in the sternplanes cycling so quickly that the optimal pitch angle for the commanded dive is never quite achieved causing the depth response to be not as good for the $\lambda=.06$ weighting. This, in analogy with linear systems, is exhibiting characteristics of being slightly overdamped. So little moment is generated by the sliding controller after the initial sternplane transient that the optimal pitch angle is not obtained. 
Forward Speed - Pitch Weight=.06
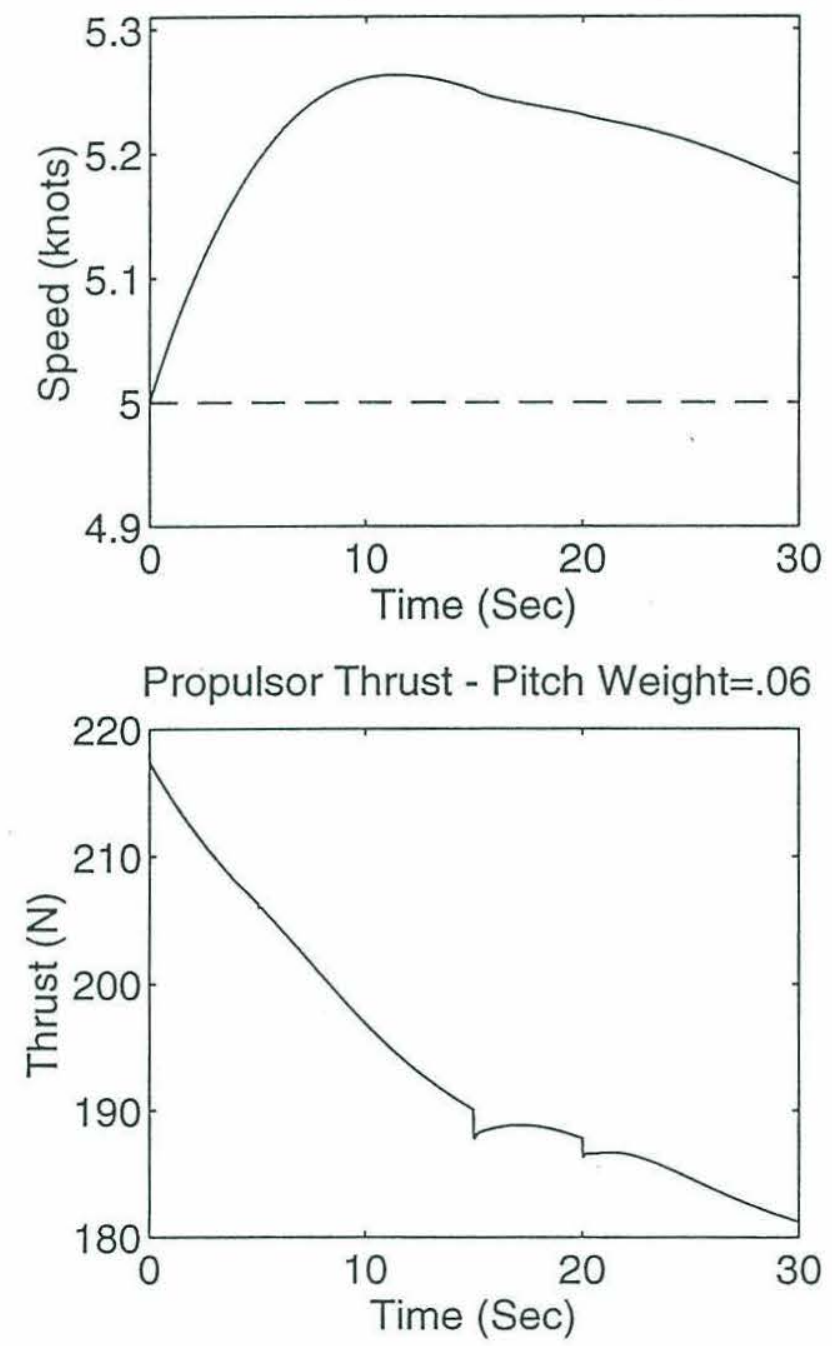

Forward Speed - Pitch Weight=.04
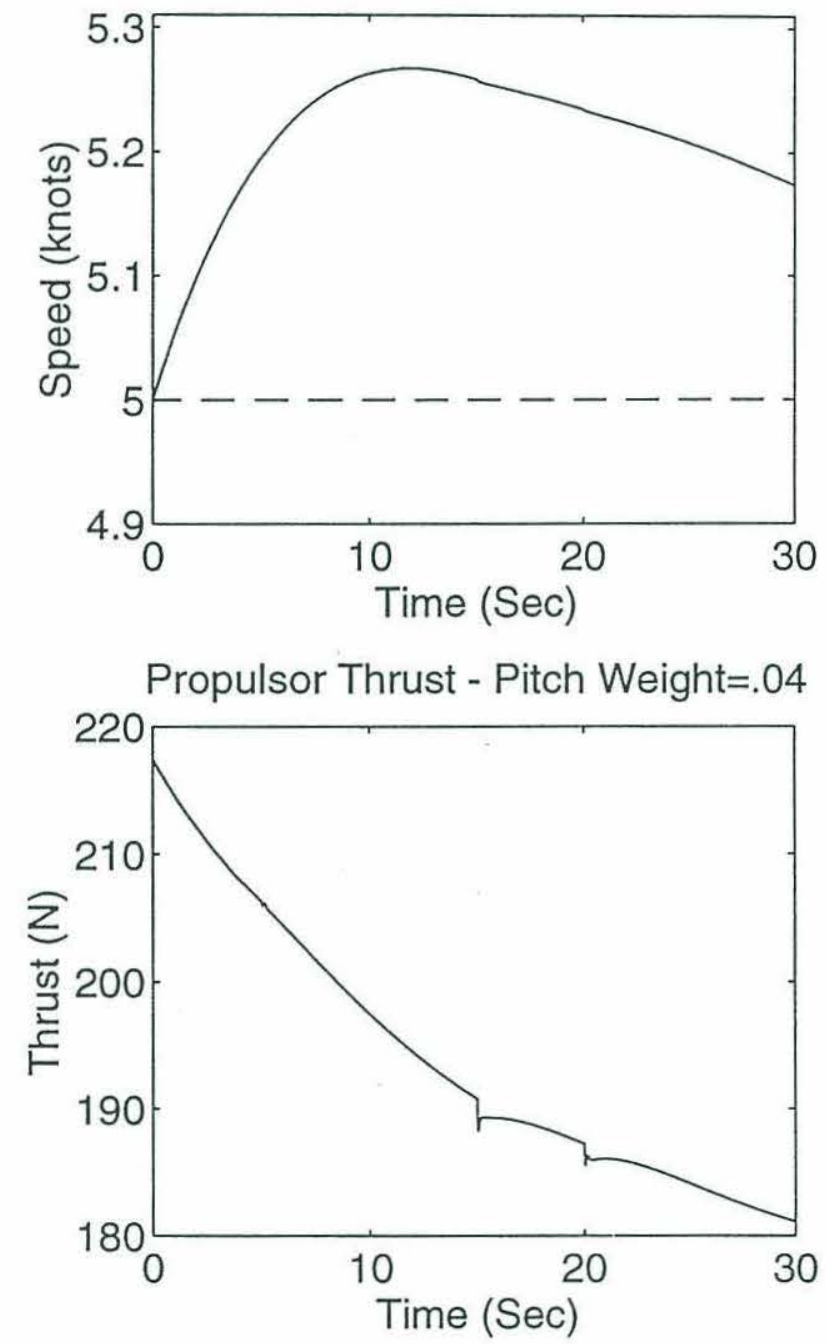

Figure 4.8 - Comparison of Forward Speed and Thrust Responses in a Commanded Dive for Pitch Weights of $\lambda=.06$ and $\lambda=.04$. Similar responses for both pitch error weightings. See figure 4.4. 


\subsubsection{Effect of Vehicle Speed on Optimal $\lambda_{i}$ 's}

The shape of vehicle depth response appears to be little influenced by the speed of the commanded trajectory for a given $\lambda$ weighting. 

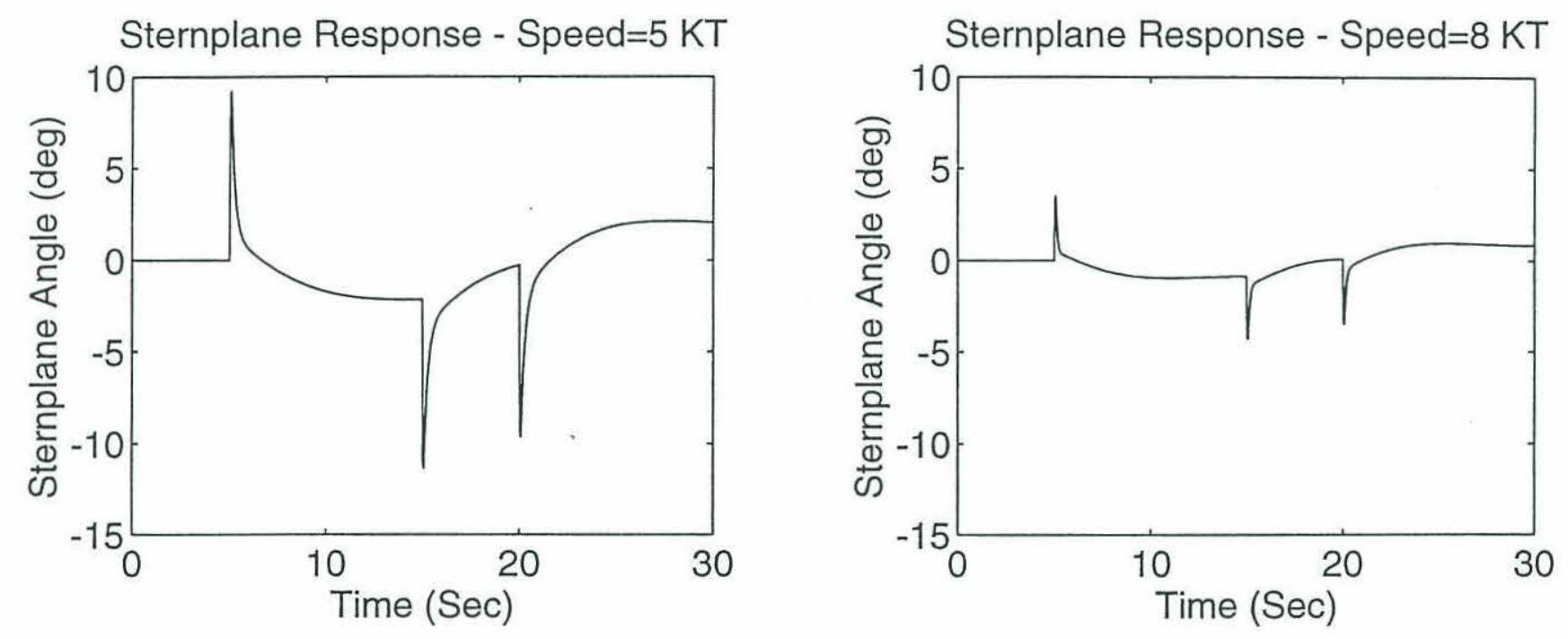

Figure 4.9 - Comparison of Sternplane Response in a Commanded Dive at 5 and 8 Knots Nominal Speed for a Pitch Weighting of $\lambda=.06$. The increased speed increases the authority of all control surface and consequently less sternplane response is required at $8 \mathrm{vs.} 5$ knots nominal speed. 

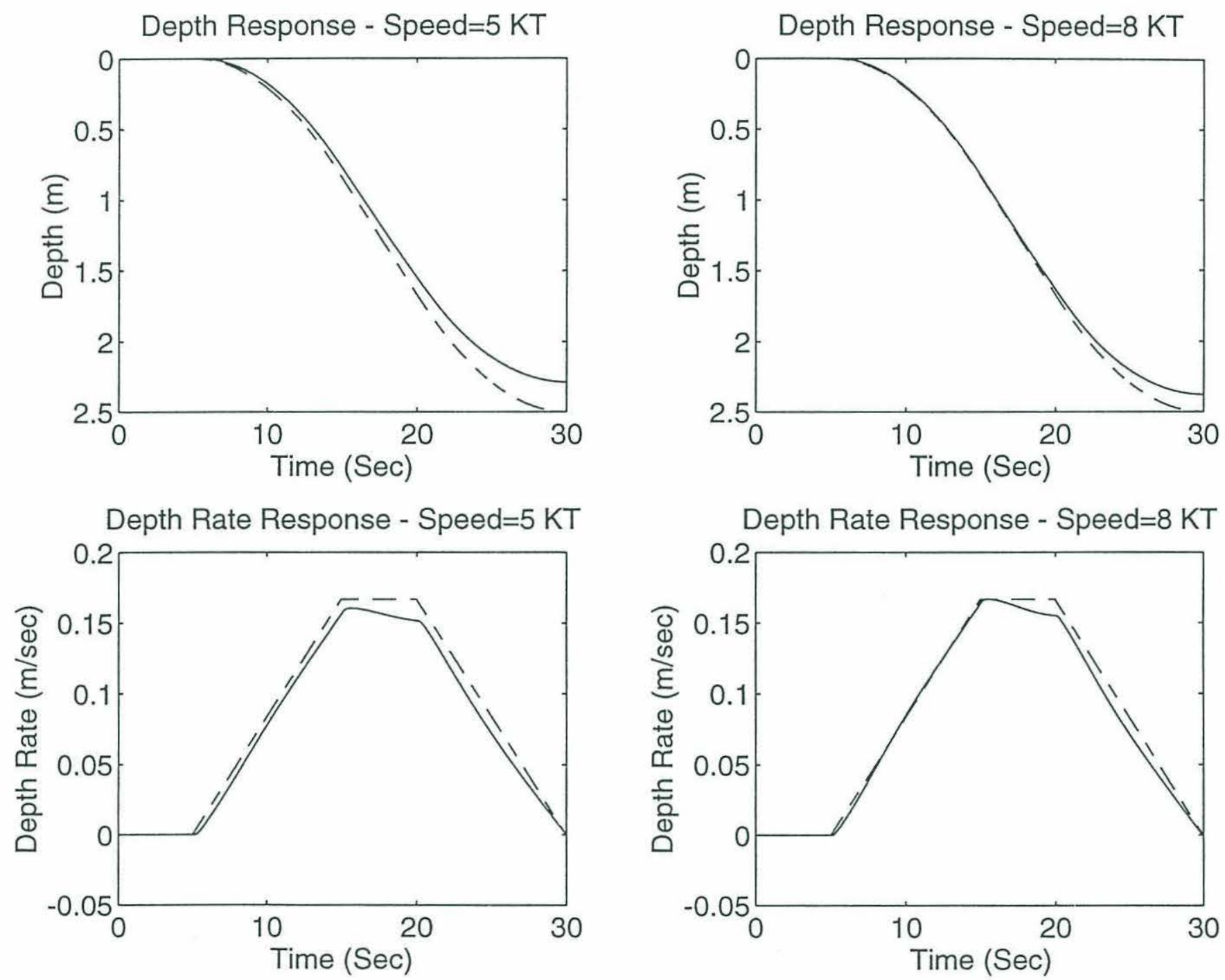

Figure 4.10 - Comparison of Depth and Depth Rate Responses in a Commanded Dive at 5 and 8 Knots Nominal Speed for a Pitch Weighting of $\lambda=.06$. Note the extremely similar response for the two speeds, an indication that the pitch weighting value is relatively independent of vehicle speed. Rather, the $\lambda$ value is better viewed as reflecting the dynamic coupling between the pitch and depth DOF's. 
Pitch Angle Response - Speed=5 KT

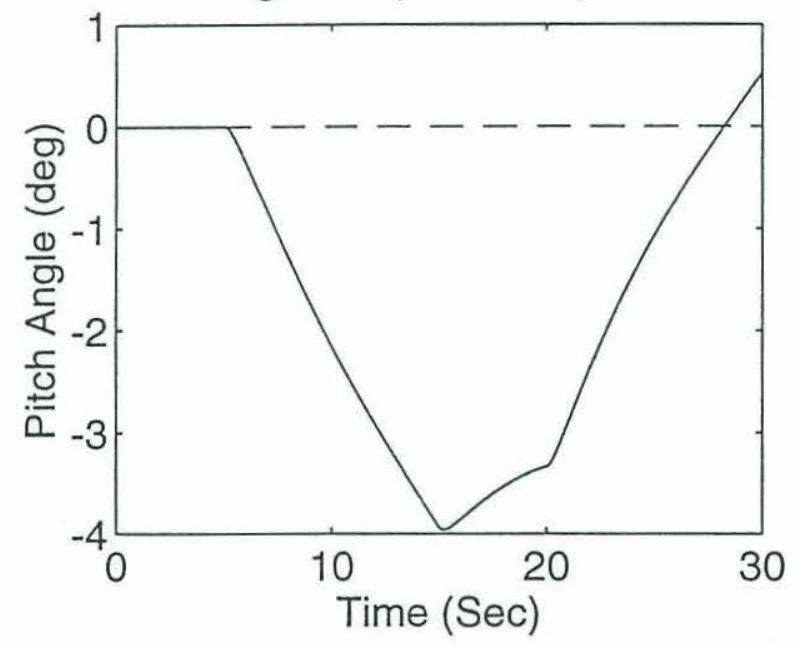

Pitch Rate Response - Speed $=5$ KT

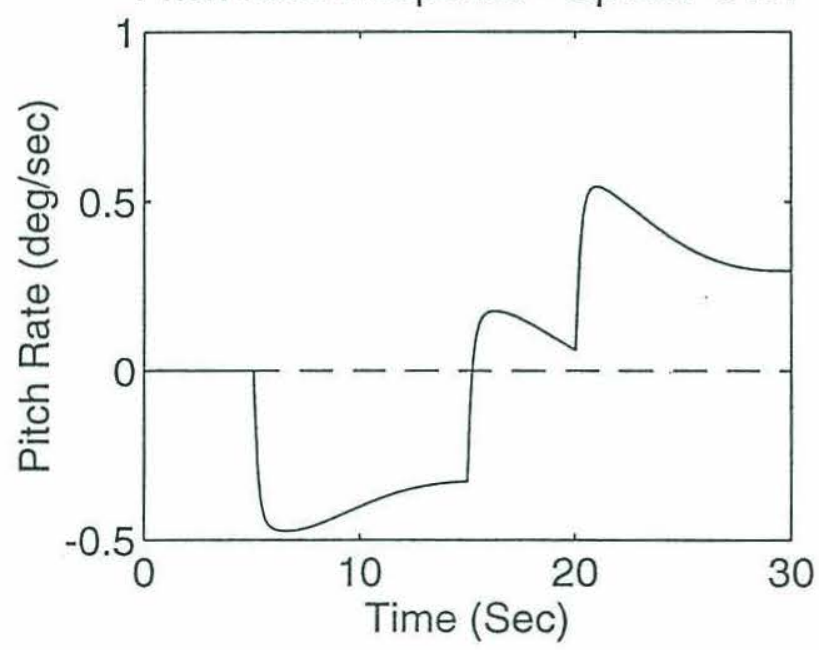

Pitch Angle Response - Speed $=8$ KT

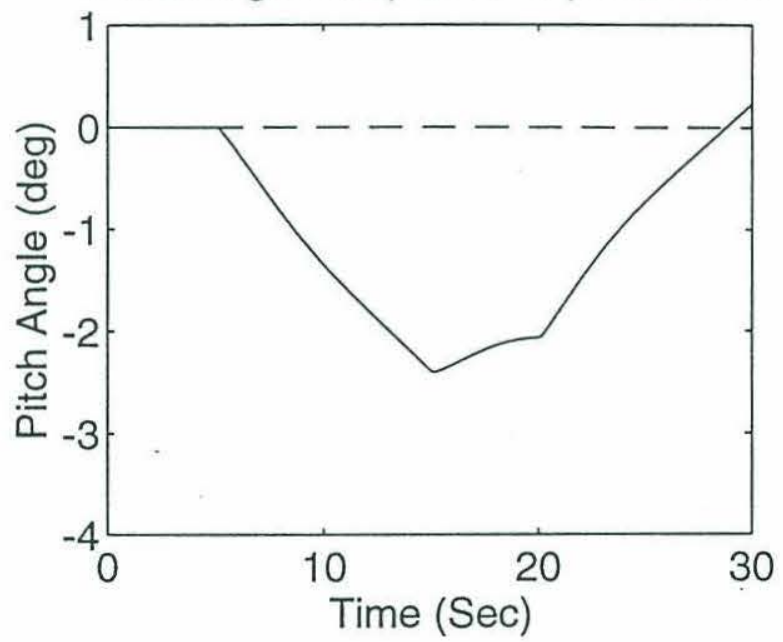

Pitch Rate Response - Speed $=8$ KT

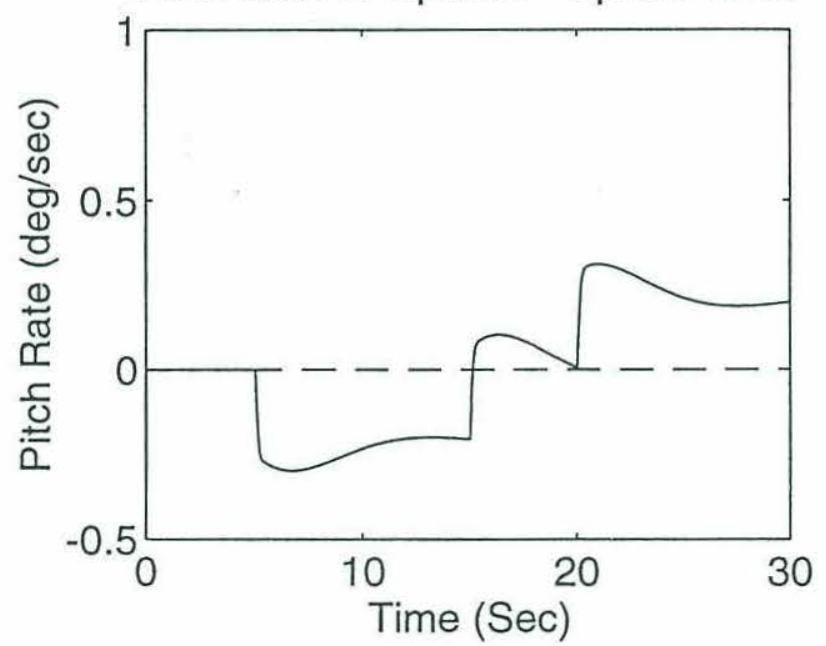

Figure 4.11 - Comparison of Pitch and Pitch Rate Responses in a Commanded Dive at 5 and 8 Knots Nominal Speed for a Pitch Weighting of $\lambda=.06$. Similarly shaped responses; naturally at 8 knots less pitch angle is needed to generate the commanded depth change. 

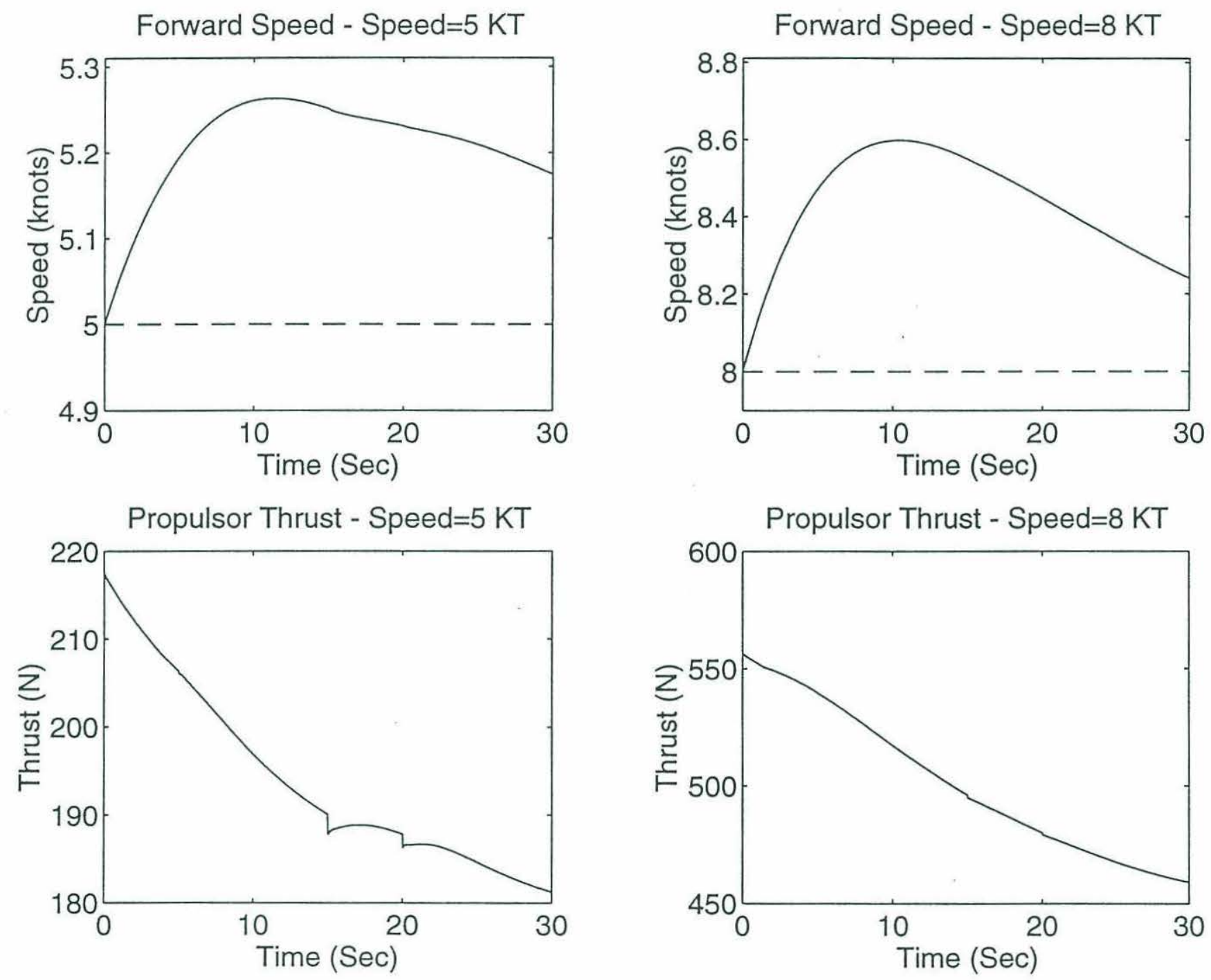

Figure 4.12 - Comparison of Forward Speed and Thrust Responses in a Commanded Dive at 5 and 8 Knots Nominal Speed for a Pitch Weighting of $\lambda=.06$. Similarly shaped responses, though the 8 knot response approaches the commanded speed faster due to the increased forward drag (from $X_{\left.u\right|_{u} \mid}$ hydrodynamic coefficient). 


\subsubsection{Effect of Trajectory Severity on Optimal $\lambda_{i}$ 's}

The shape of vehicle depth response appears to be little influenced by the severity

of the commanded trajectory for a given $\lambda$ weighting - further evidence that the optimal $\lambda$ is a direct consequence of the underlying dynamics relating pitch and depth. 

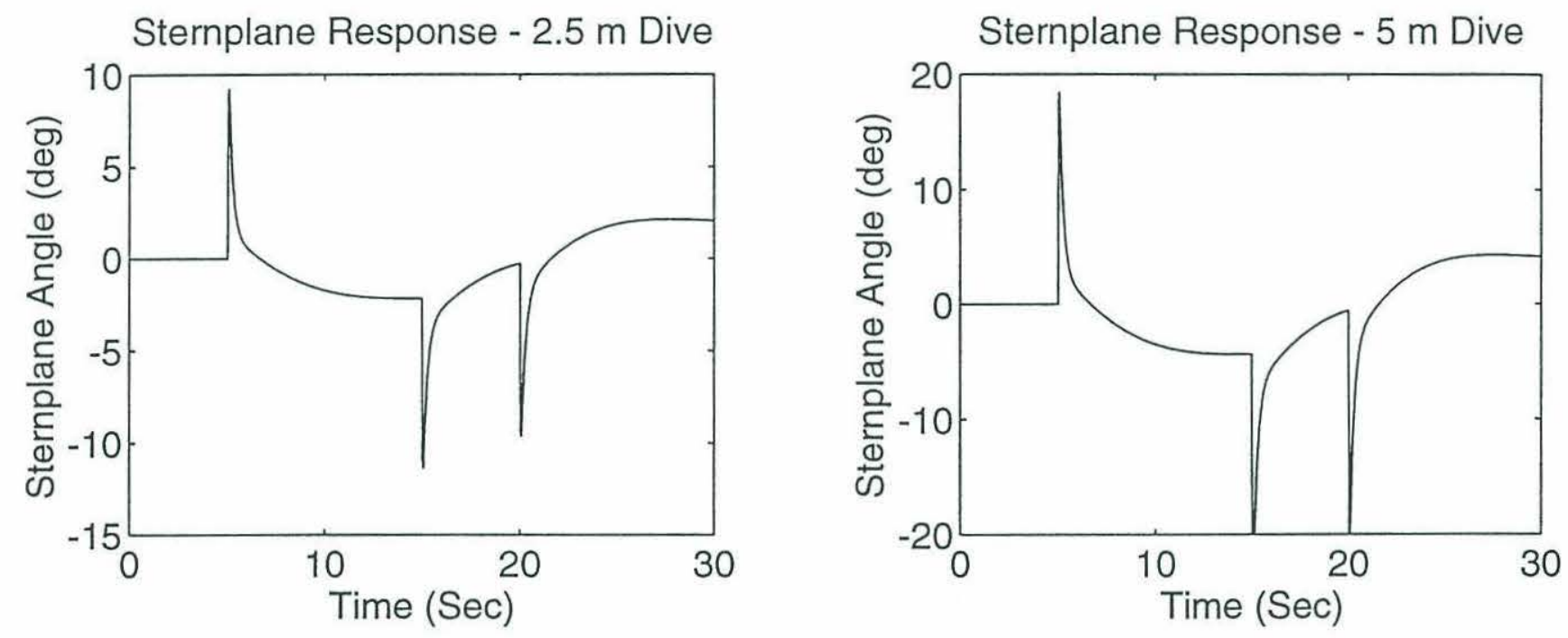

Figure 4.13 - Comparison of Sternplane Response in Commanded Dives of 2.5 and 5 Meters at 5 Knots Nominal Speed for a Pitch Weighting of $\lambda=.06$. Similarly shaped responses; naturally, approximately twice the sternplane angle is required to effect twice the depth change in equal amounts of time. 

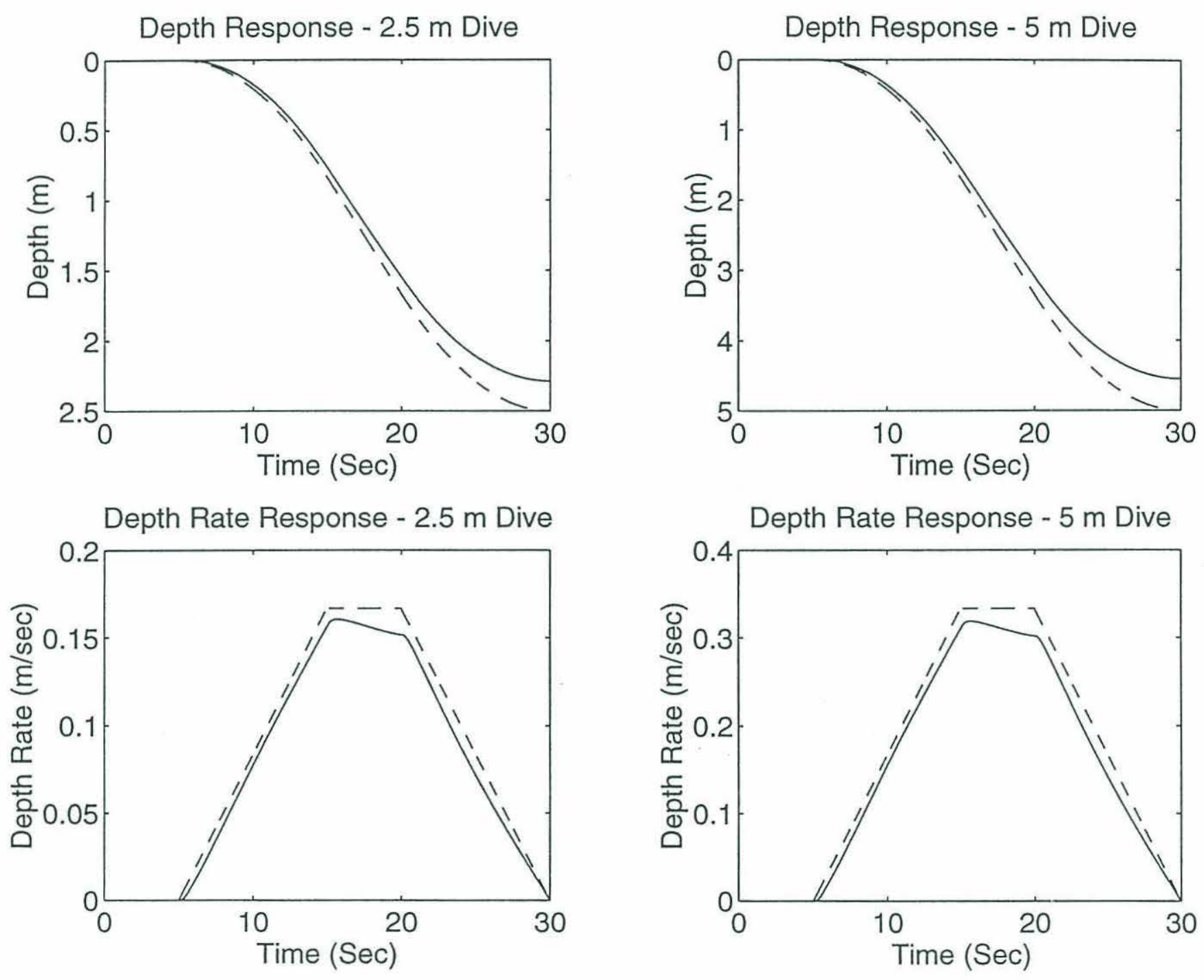

Figure 4.14 - Comparison of Depth and Depth Rate Responses in Commanded Dives of 2.5 and 5 Meters at 5 Knots Nominal Speed for a Pitch Weighting of $\lambda=.06$. Note the extremely similarly shaped responses for the two commanded depth changes, another indication that the pitch weighting value purely reflects the dynamic coupling between the pitch and depth DOF's. 


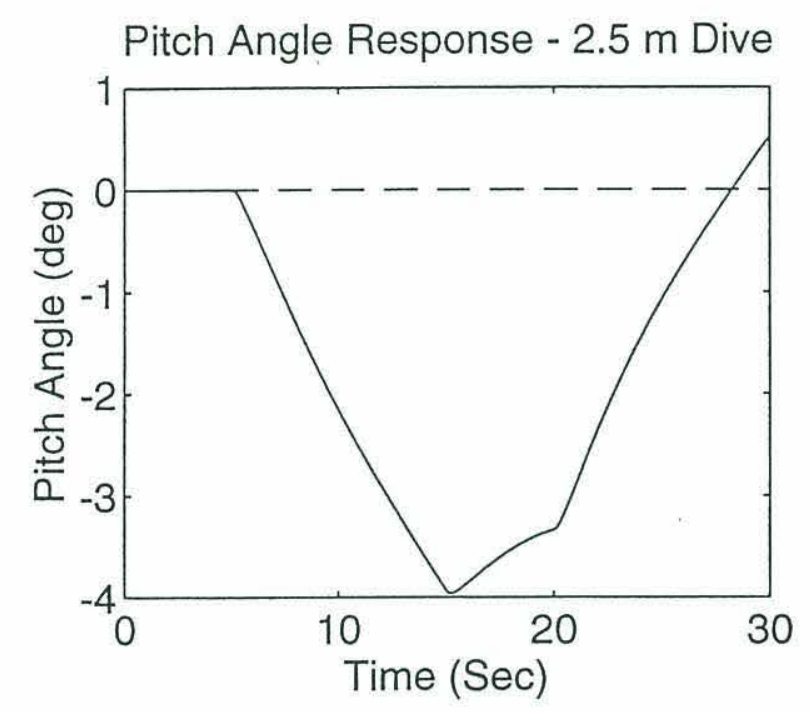

Pitch Rate Response - 2.5 m Dive

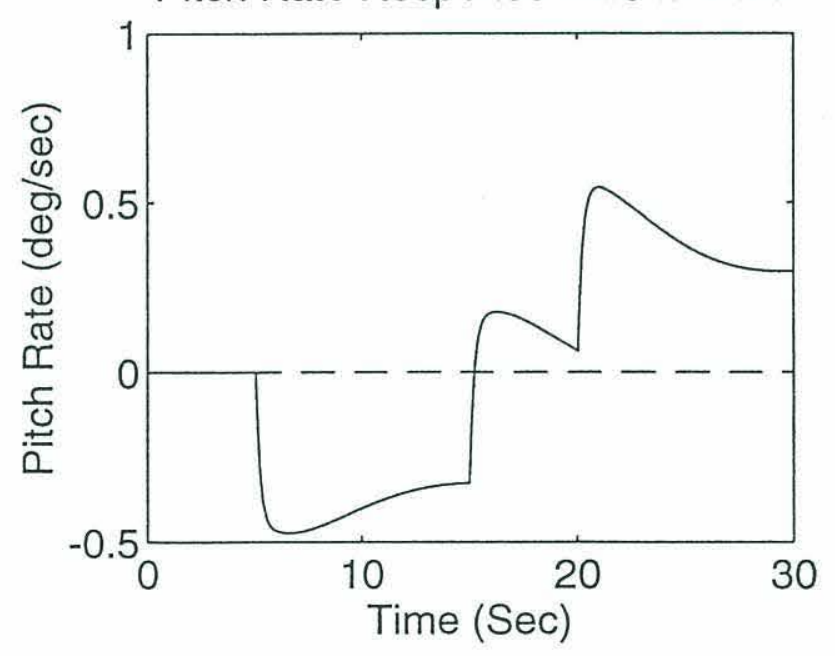

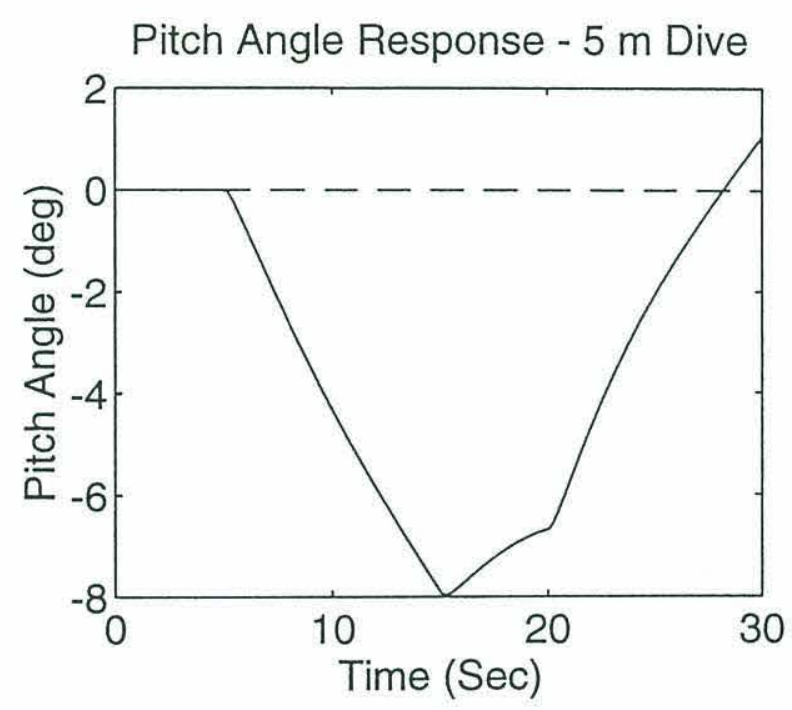

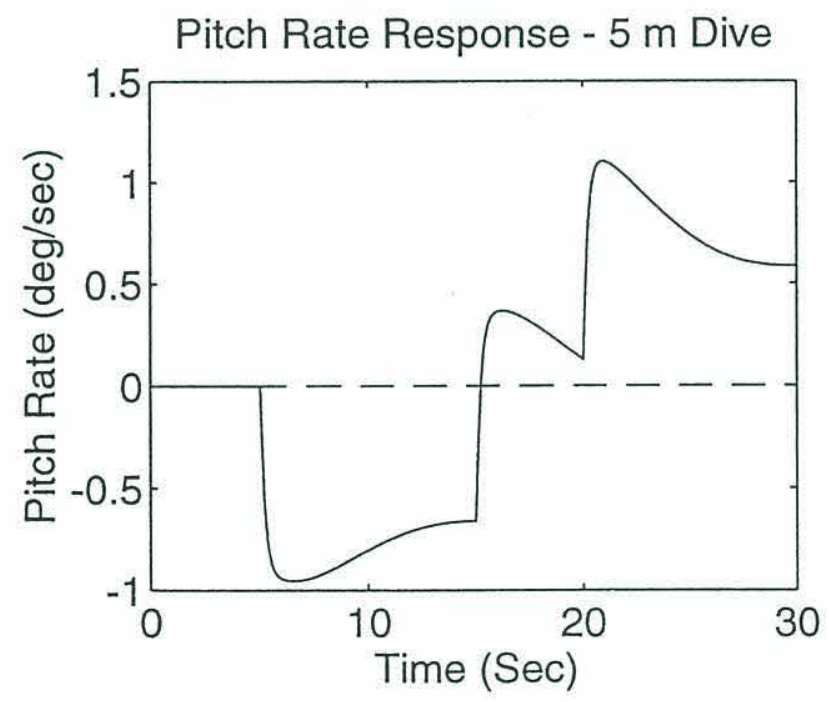

Figure 4.15 - Comparison of Pitch and Pitch Rate Responses in Commanded Dives of 2.5 and 5 Meters at 5 Knots Nominal Speed for a Pitch Weighting of $\lambda=.06$. Similarly shaped responses - see figure 4.14 . 

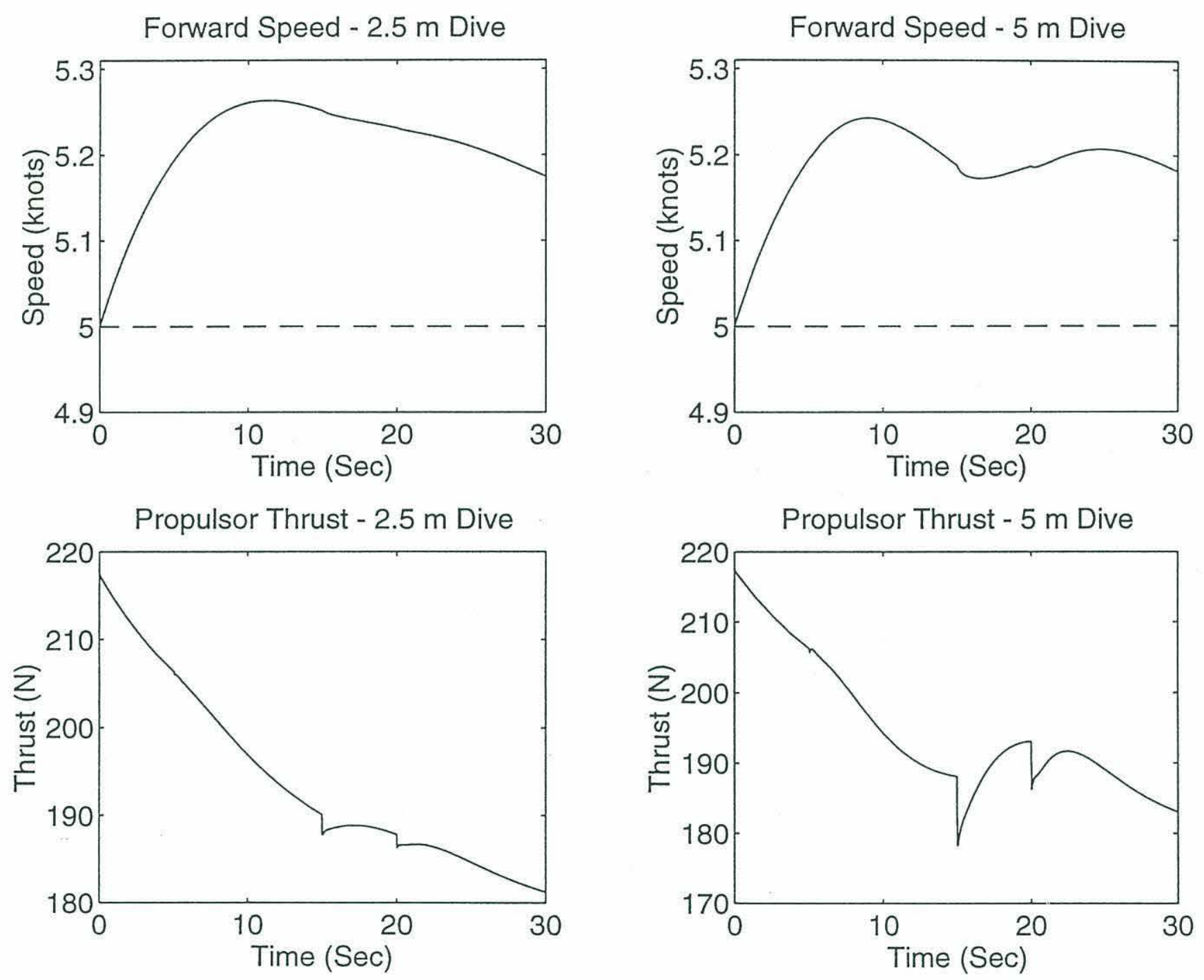

Figure 4.16 - Comparison of Forward Speed and Thrust Responses in Commanded Dives of 2.5 and 5 Meters at 5 Knots Nominal Speed for a Pitch Weighting of $\lambda=.06$. Similarly shaped responses, as expected from the previous results. 


\subsubsection{Vehicle Roll Capability}

As evidenced in the following plots, the ailerons do not provide enough control authority to control roll during a commanded roll maneuver. Fortunately, this is not a particularly desirable trajectory - it is much more important that the vehicle not roll during turns and/or depth changes. This lack of control authority made the weighting of this the roll DOF rather moot $-\lambda_{K}$ was set at 1 . 
Desired and Actual Roll Angle
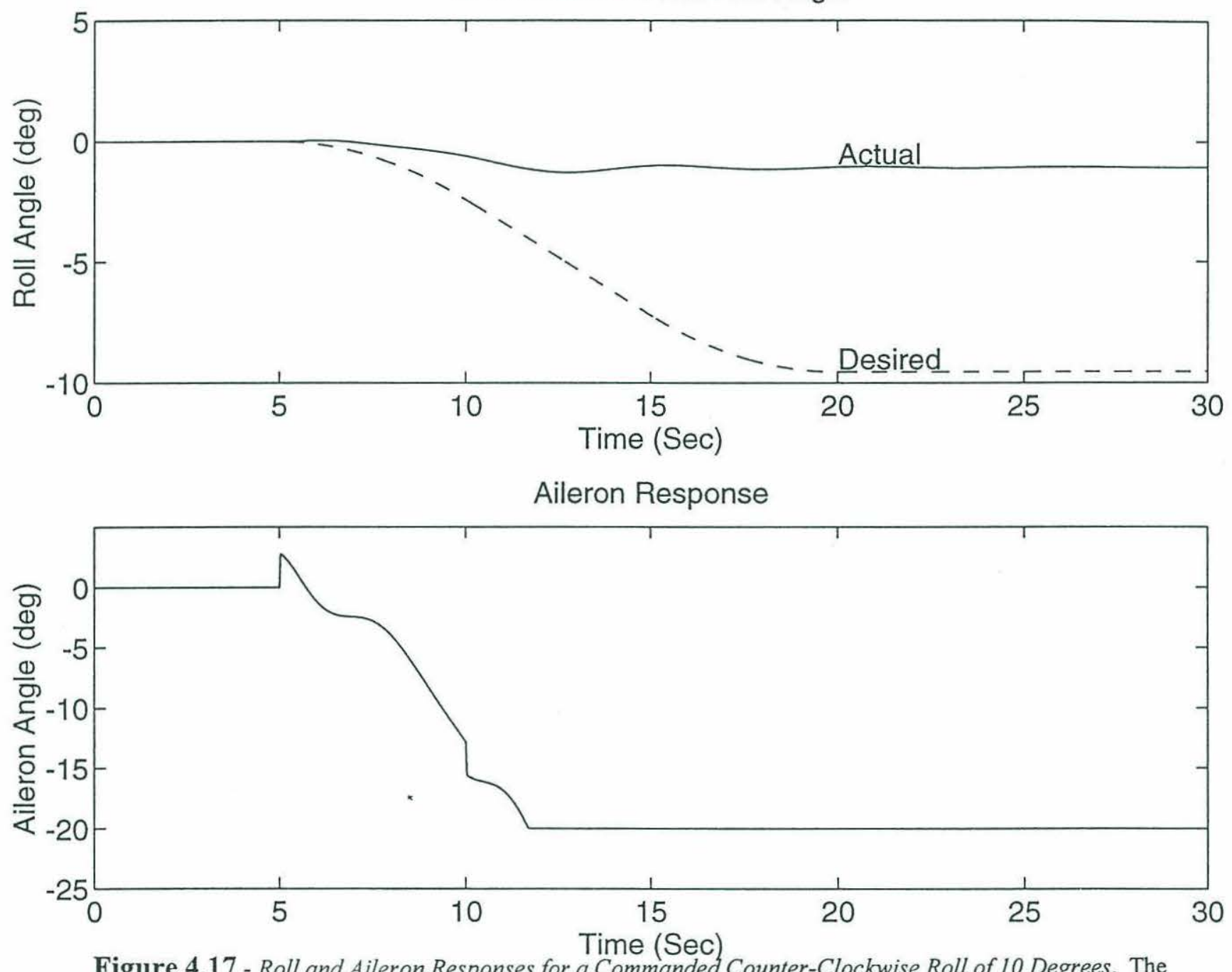

Figure 4.17 - Roll and Aileron Responses for a Commanded Counter-Clockwise Roll of 10 Degrees. The vehicle has great difficulty executing this commanded maneuver due primarily to the low control authority in this degree of freedom (due to the small value of $K_{p}$ ) - note the saturated control surface - but also to the restoring force incurred by the assumed $\mathrm{z}_{\mathrm{G}}-\mathrm{z}_{\mathrm{B}}$ separation of $.005 \mathrm{~m}$ and the simulated $10 \mathrm{~N}$ positive buoyancy. Fortunately, it is difficult to conceive when a vehicle like the 21 UUV would require such a maneuver; it is much more important that the vehicle avoid rolling in the presence of disturbances or during more typical maneuvers such as trackline changes. Also note the non-minimum phase response of the ailerons in the initial stages of the commanded roll. 

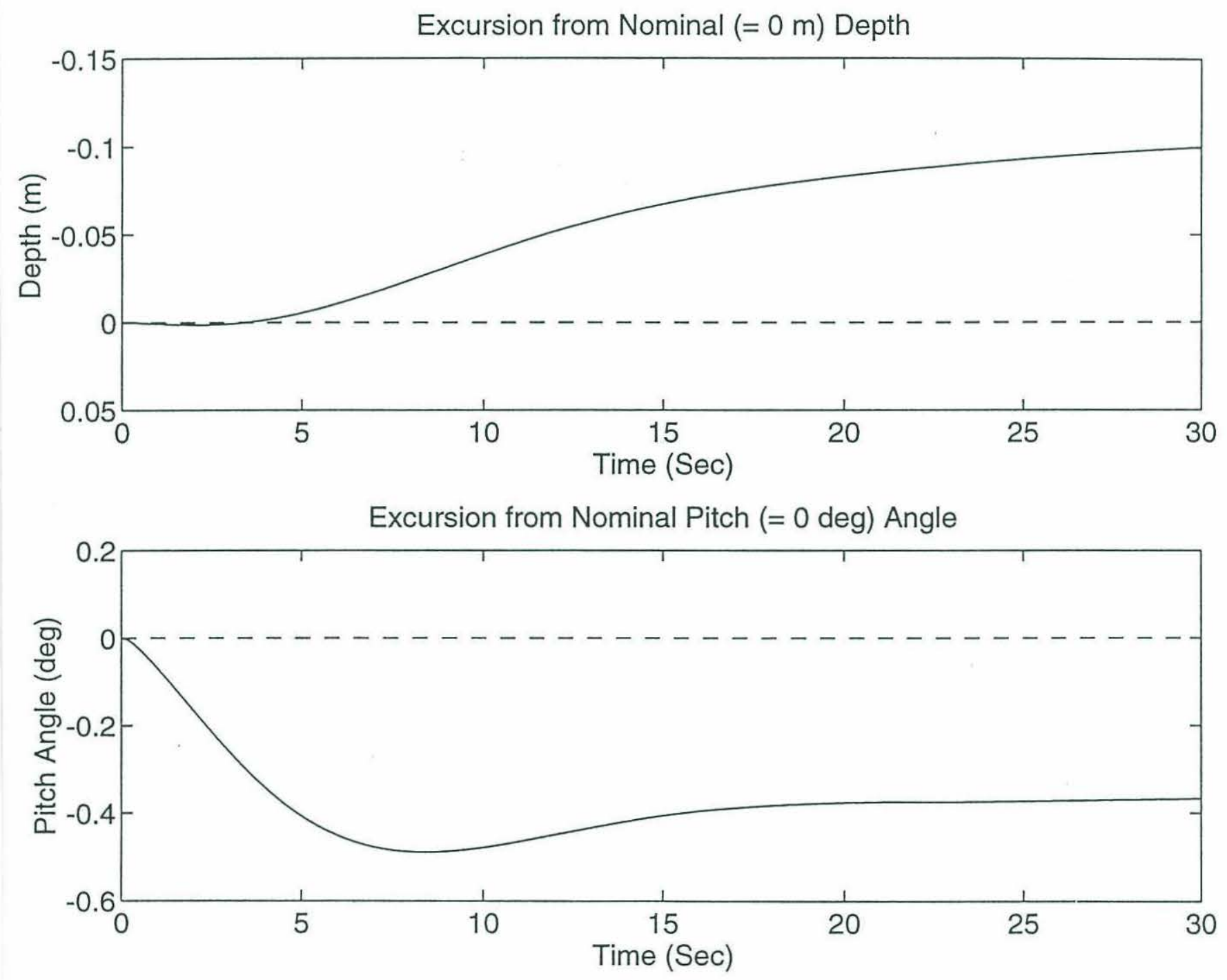

Figure 4.18 - Depth and Pitch Angle Responses for a Commanded Counter-Clockwise Roll of 10 Degrees. Mostly a result of the $10 \mathrm{~N}$ positive buoyancy; but there is slight coupling from the roll DOF. 

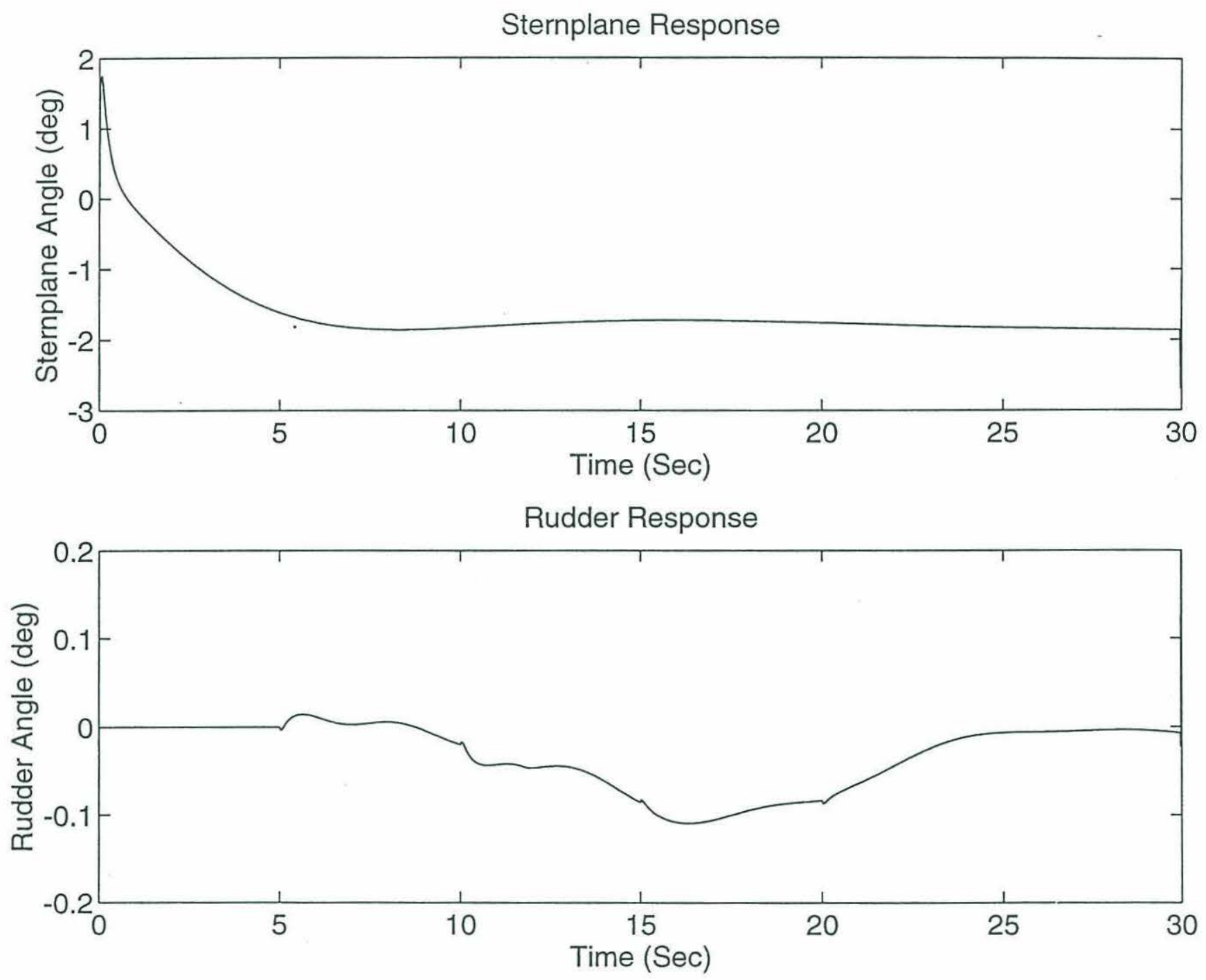

Figure 4.20 - Sternplane and Rudder Responses for a Commanded Counter-Clockwise Roll of 10 Degrees. The sternplane response is mainly a result of the positive buoyancy, but both control surfaces contribute a bit to the control of the roll angle. 


\subsubsection{Vehicle Coordinated Maneuvering Response}

To test the vector sliding controller out in the 4 DOF available control mode, simulations were performed with coordinated trajectories (e.g. dive and trackline change simaltaneously). As is seen in the plots, response is excellent indicating that the controller is easily able to handle complicated and coupled maneuvers. 
Commanded Dive and Trackline Change

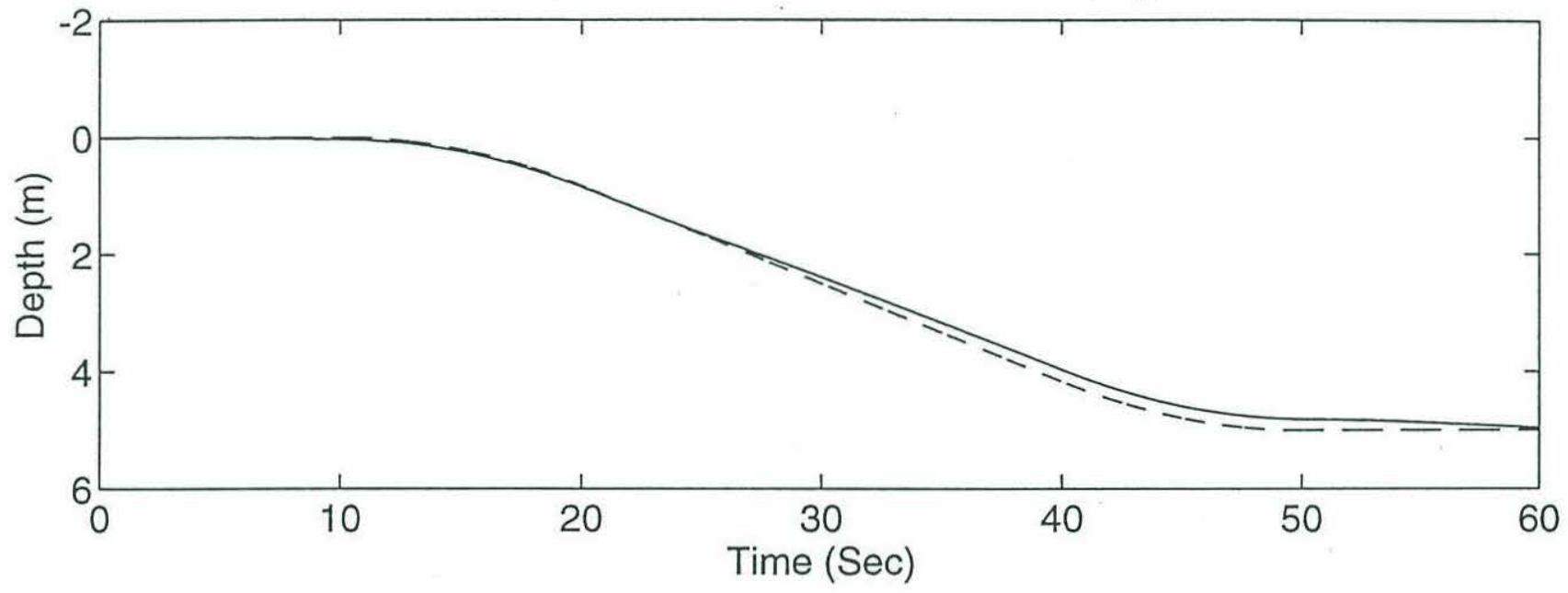

Commanded Dive and Trackline Change

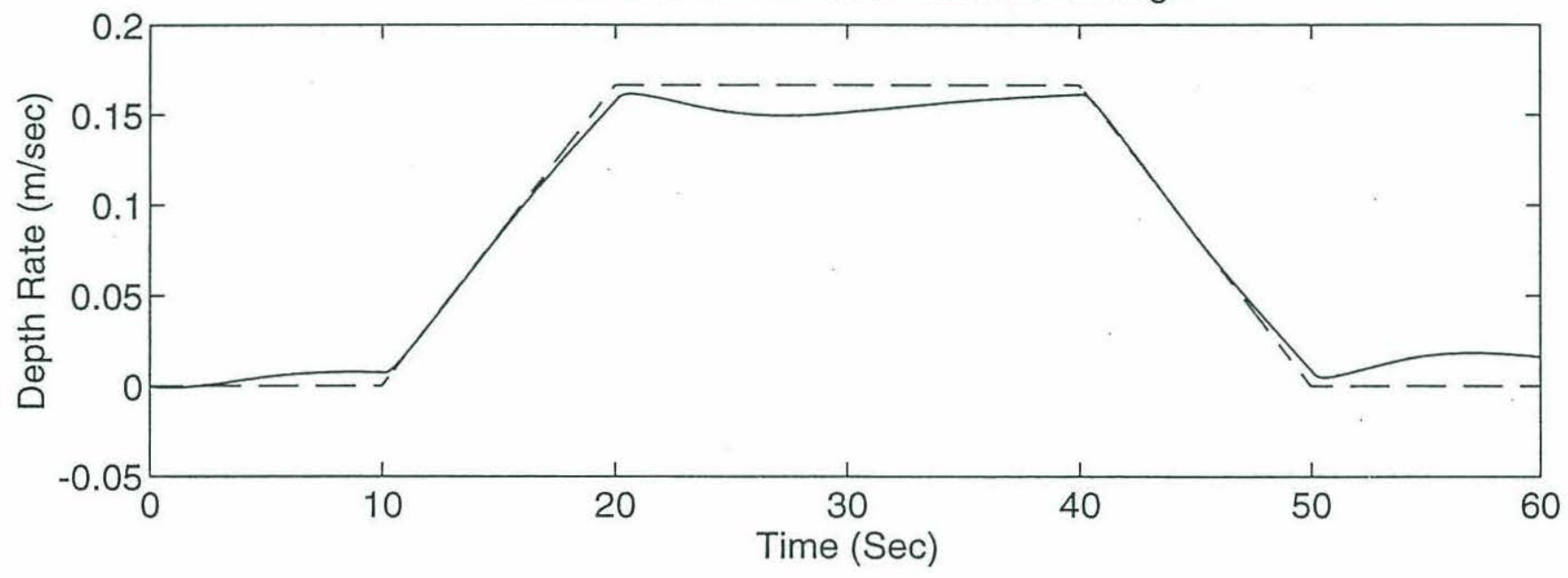

Figure 4.21 - Depth and Depth Rate for a Coordinated Dive and Trackline Maneuver with a $10 \mathrm{~N}$ Positive Buoyancy. The $4 \mathrm{DOF}$ implementation of the $6 \mathrm{DOF}$ controller provides excellent control in this coupled maneuver. 


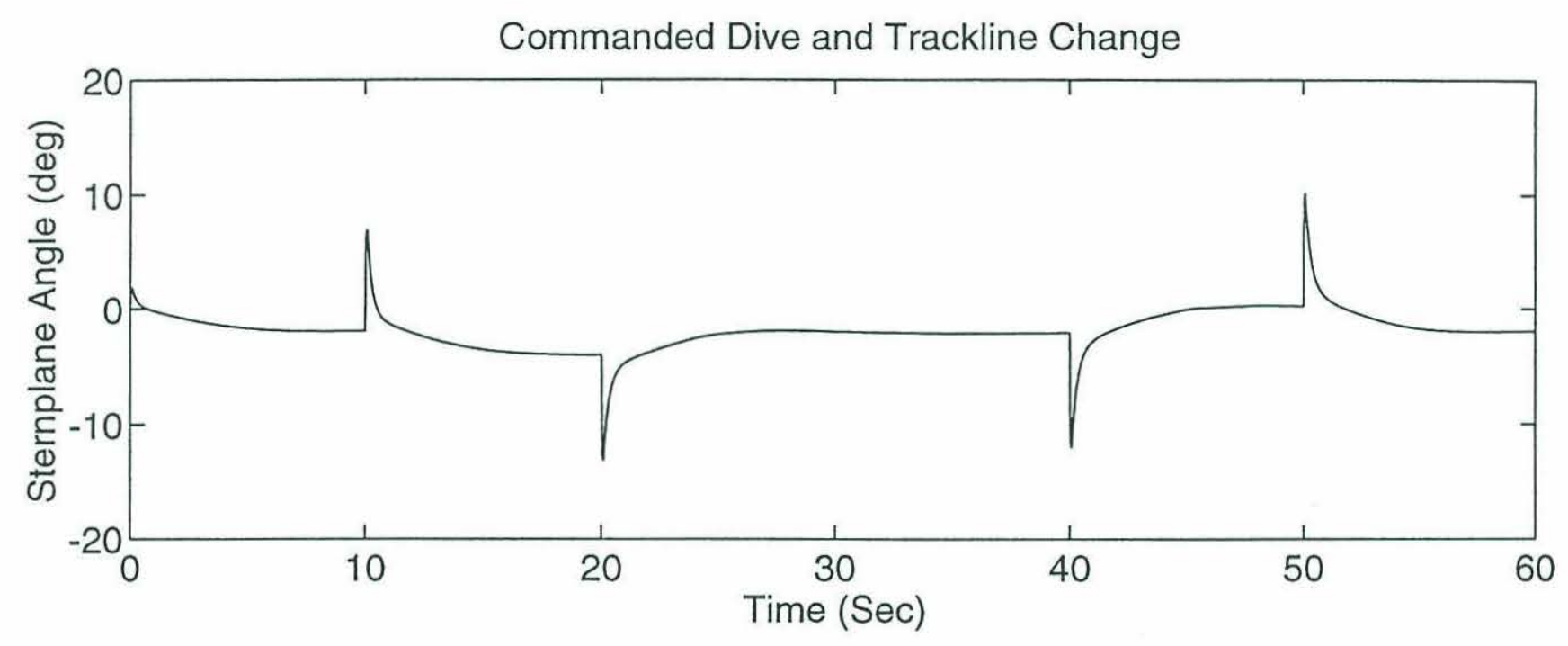

Commanded Dive and Trackline Change

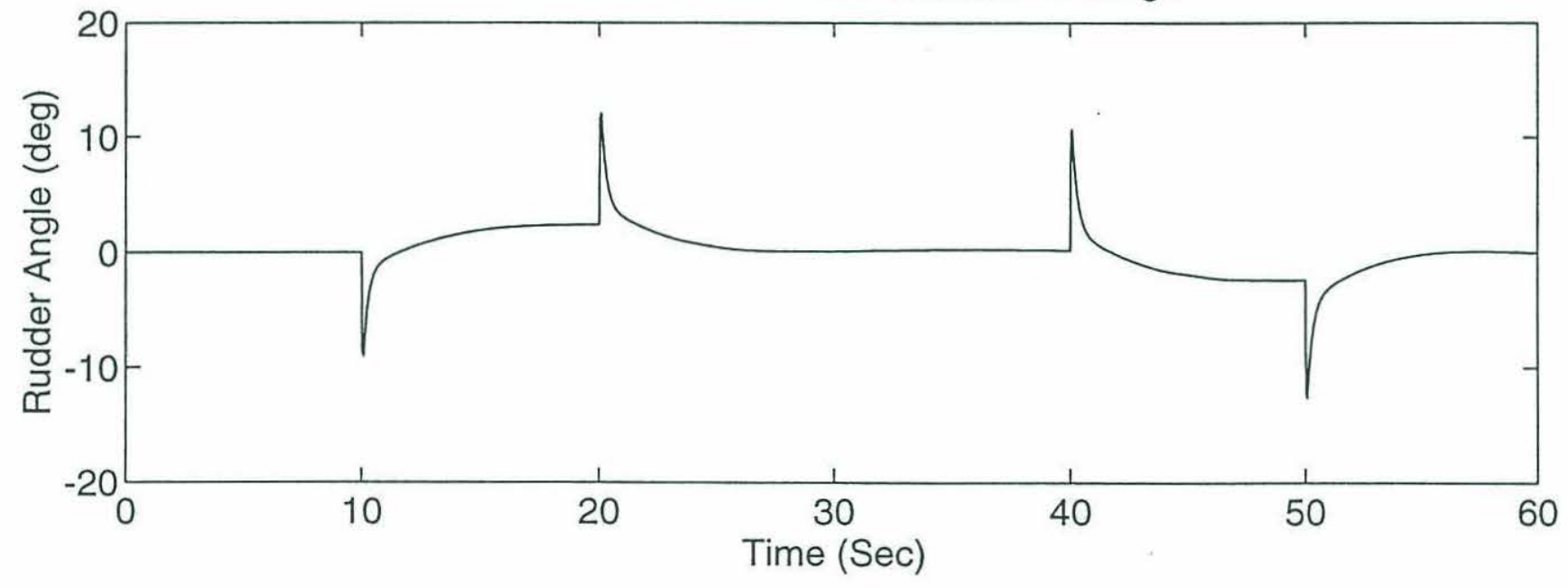

Figure 4.23 - Sternplane and Rudder Responses for a Coordinated Dive and Trackline Maneuver with a $10 \mathrm{~N}$ Positive Buoyancy. As expected from the symmetry about the $y$-and $z$-axes, control surface actions are similar (though in different directions due to the chosen coordinated maneuver). Note the non-minimum phase actions of the control surfaces - first get the requisite pitch and yaw angles, respectively, then allow for the depth and trackline changes. 

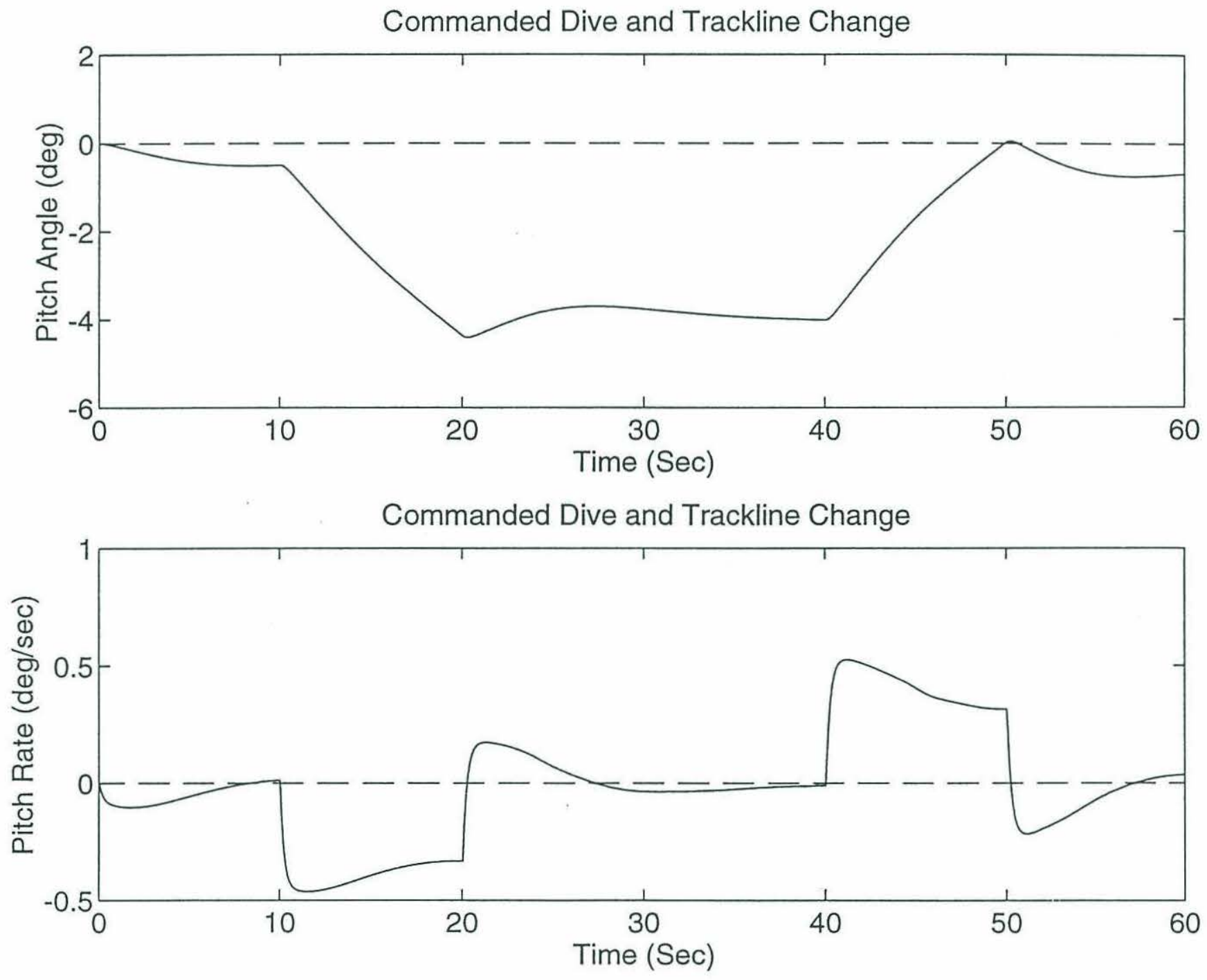

Figure 4.24- Pitch and Pitch Rate for a Coordinated Dive and Trackline Maneuver with a 10 N Positive Buoyancy. Corresponds to depth and depth rate responses. 

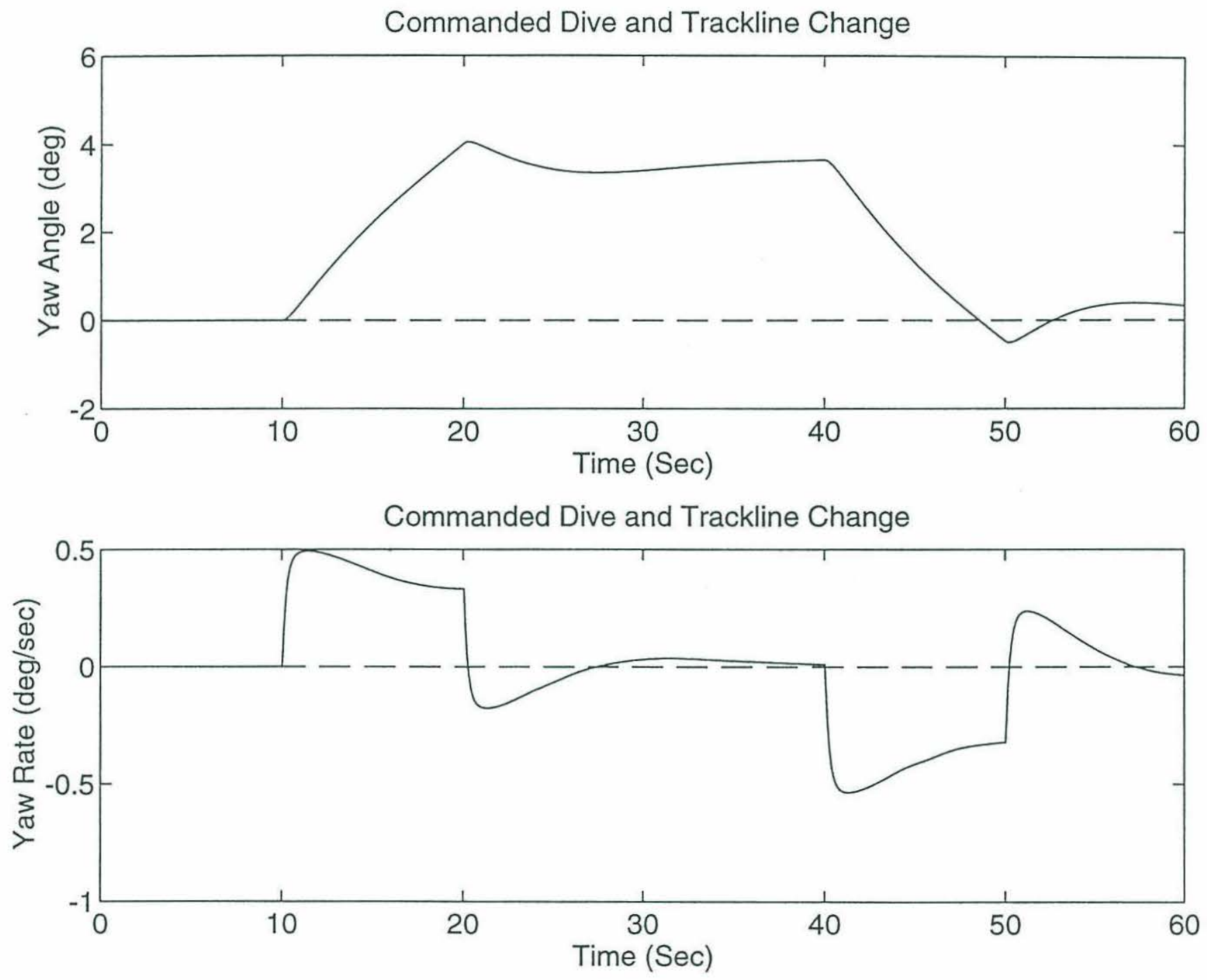

Figure 4.25- Yaw and Yaw Rate for a Coordinated Dive and Trackline Maneuver with a $10 \mathrm{~N}$ Positive Buoyancy. Corresponds to trackline and heading rate responses. 
Commanded Dive and Trackline Change

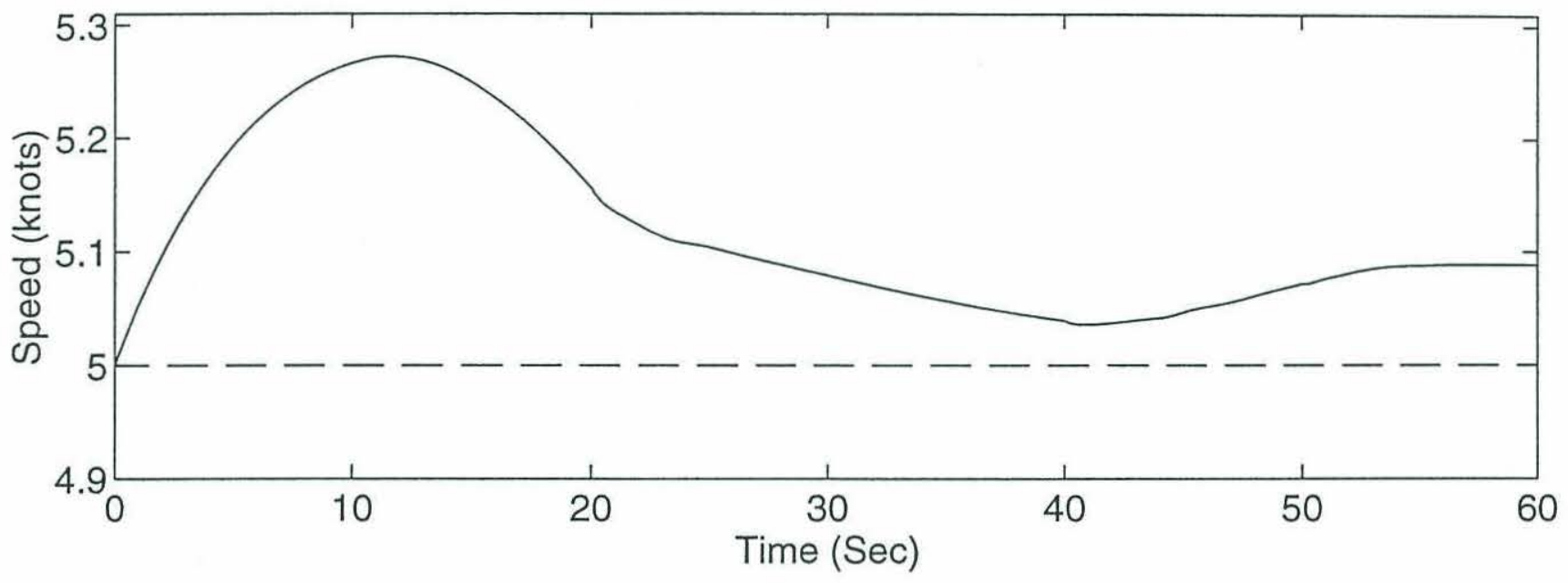

Commanded Dive and Trackline Change

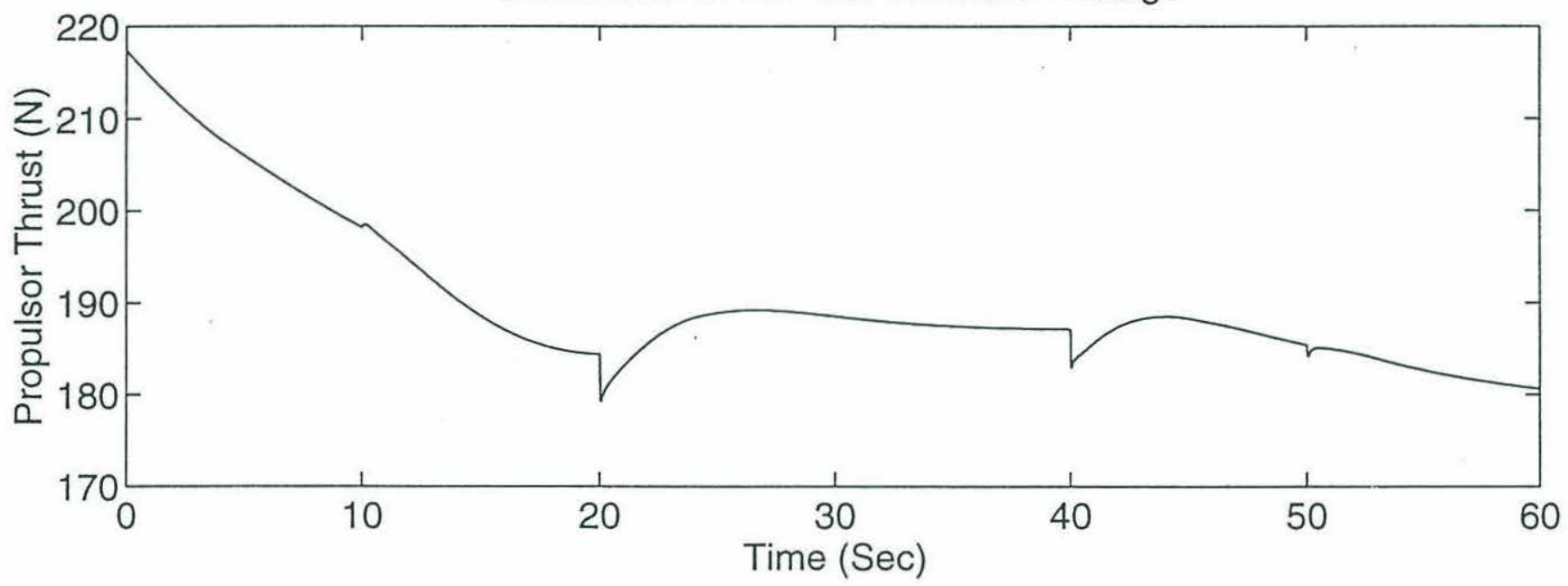

Figure 4.26- Forward Speed and Thrust for a Coordinated Dive and Trackline Maneuver with a $10 \mathrm{~N}$ Positive Buoyancy. Response as expected. 


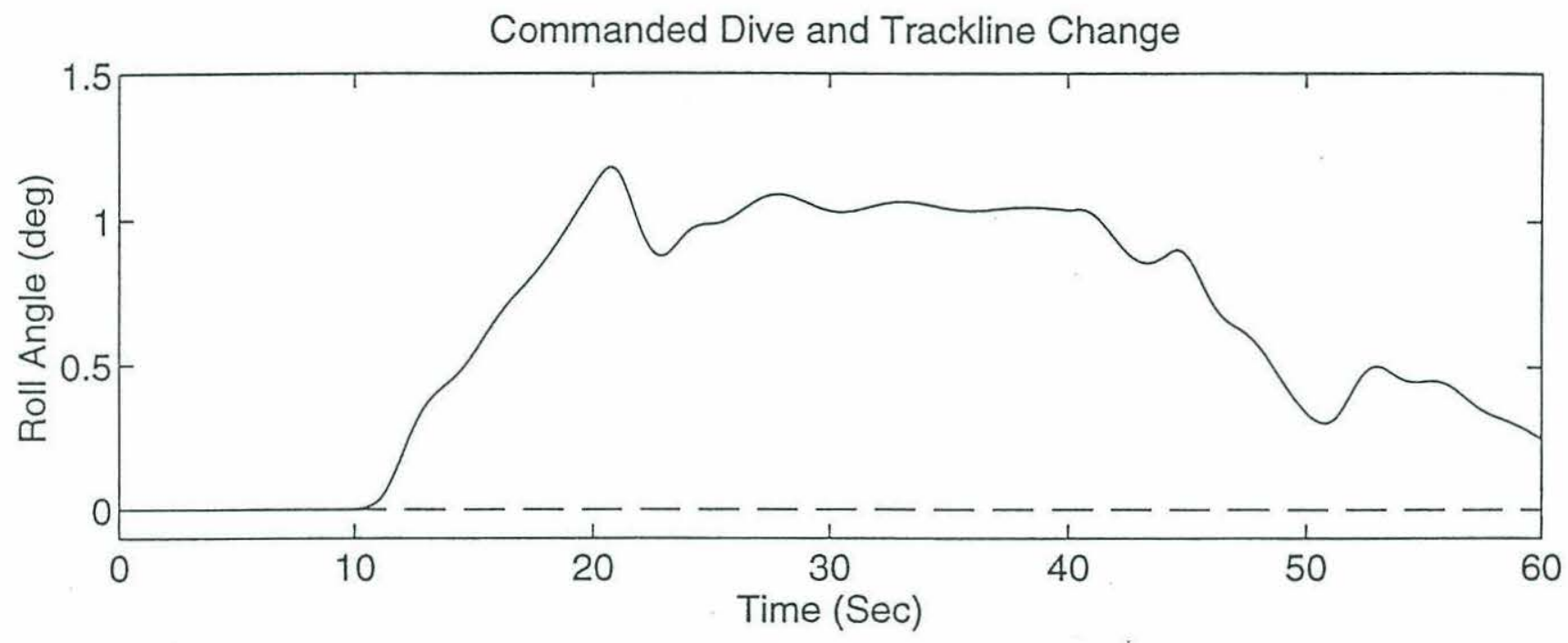

Commanded Dive and Trackline Change

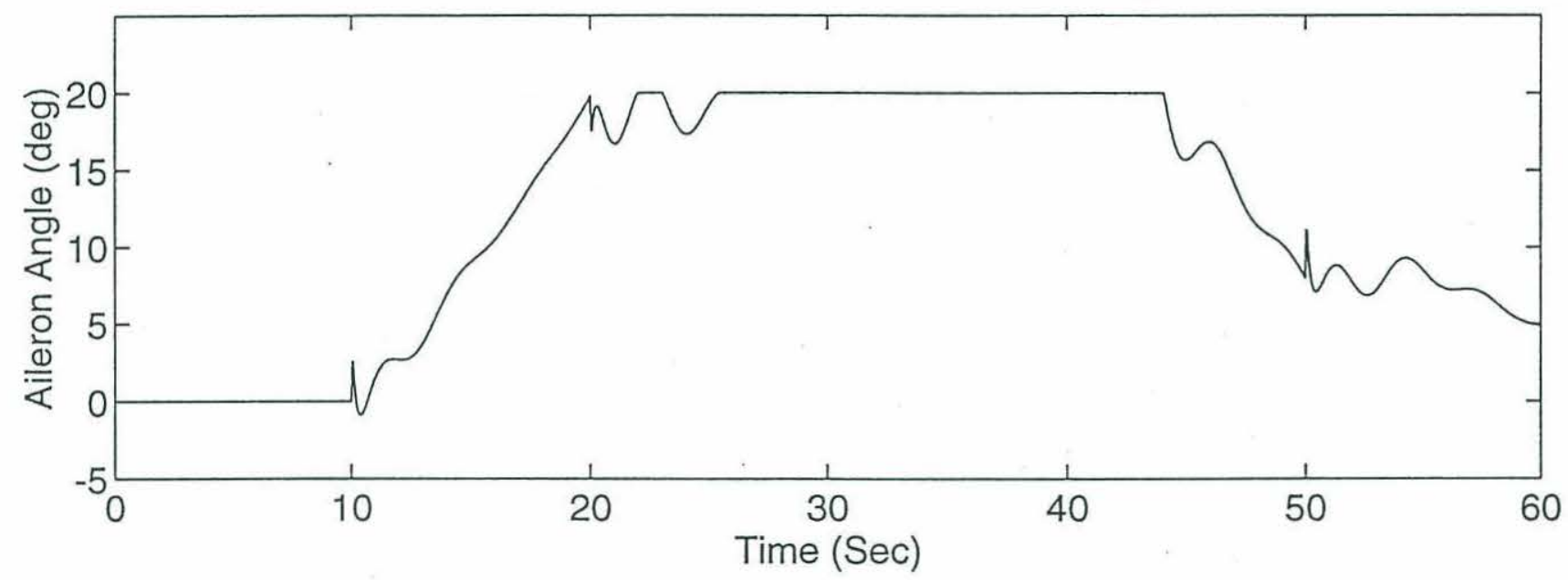

Figure 4.27- Roll and Aileron Angles for a Coordinated Dive and Trackline Maneuver with a $10 \mathrm{~N}$ Positive Buoyancy. As alluded to in figure 4.17, it is important that the 4 DOF implementation of the 6 DOF sliding controller be able to control roll response in normal commanded maneuvers (and in disturbances). Unfortunately, the low value of the hydrodynamic coefficient $K_{p}$ causes this DOF to respond like a very underdamped spring-mass system, resulting in saturated aileron response. The saving grace is the restoring force generated by the $\mathrm{z}_{\mathrm{G}}-\mathrm{z}_{\mathrm{B}}$ separation - thus the roll DOF can be thought as "stabilizable" if not actually "controllable". If this situation is deemed unacceptable corrective action - e.g. larger ailerons - is required. 
Commanded Rise and Trackline Change

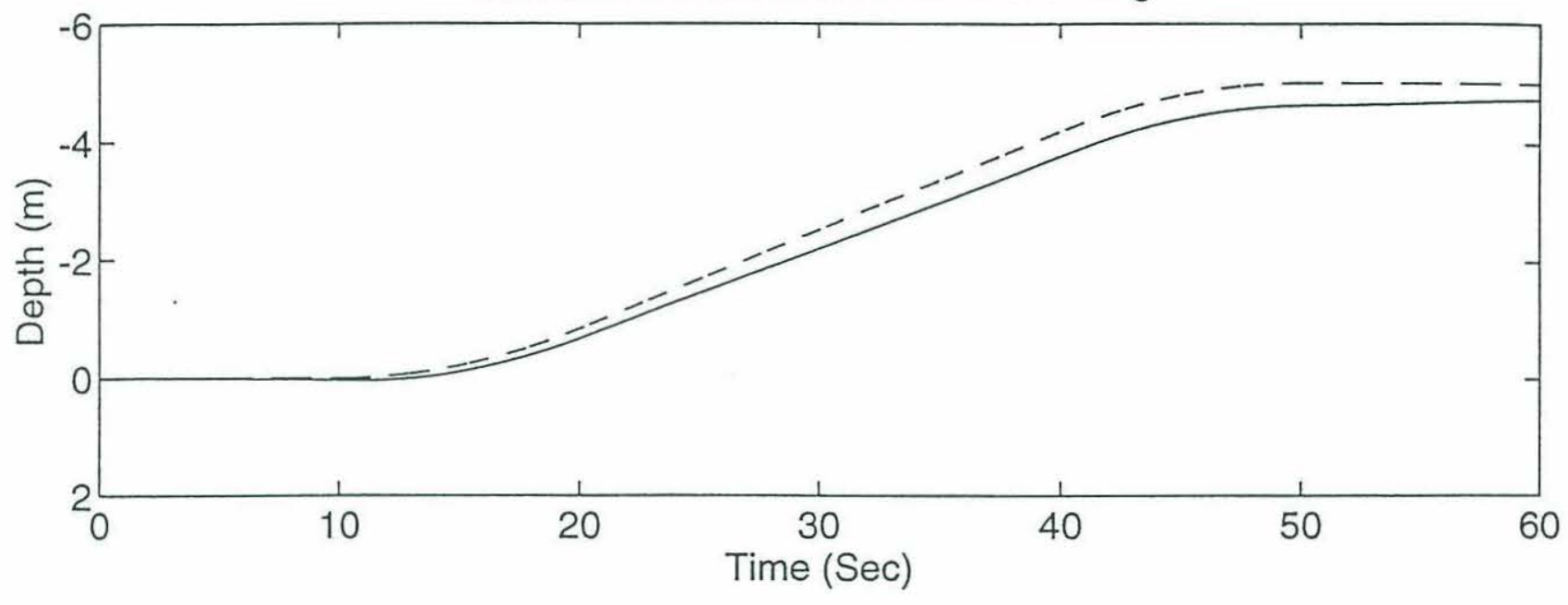

Commanded Rise and Trackline Change

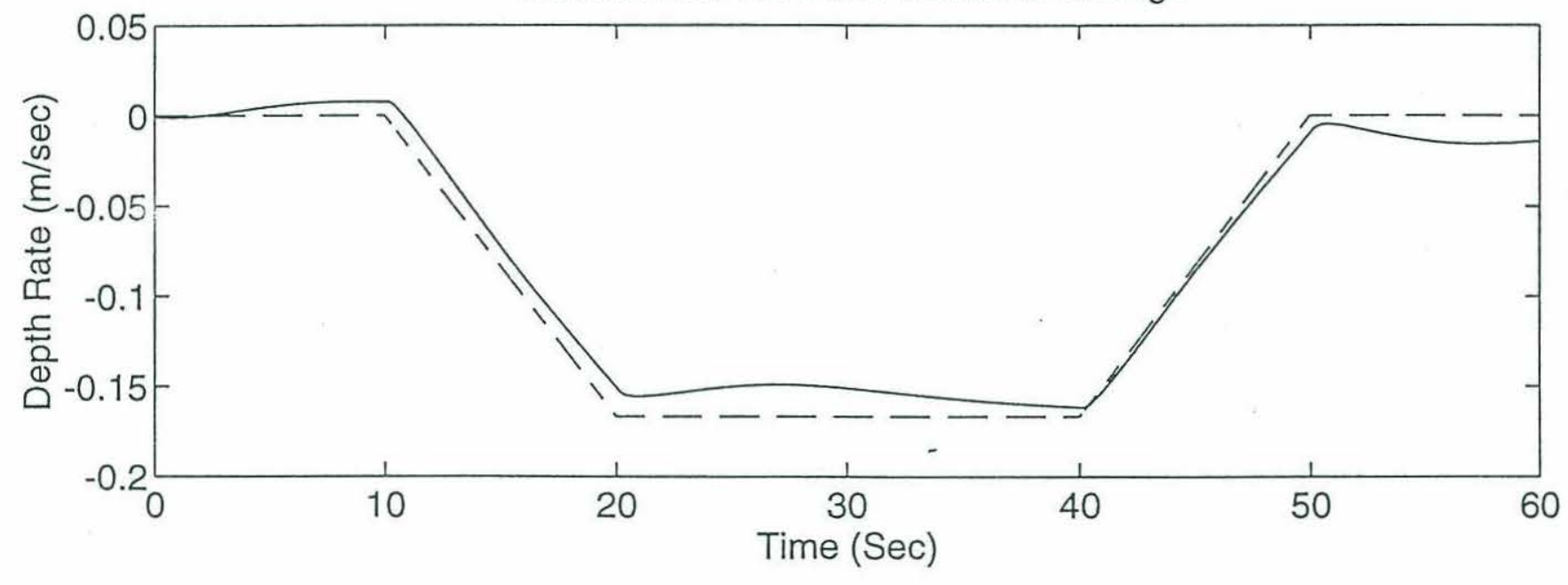

Figure 4.28 - Depth and Depth Rate for a Coordinated Rise and Trackline Maneuver with a $10 \mathrm{~N}$ Positive Buoyancy. The $4 \mathrm{DOF}$ implementation of the $6 \mathrm{DOF}$ controller provides excellent control in this coupled manuever. Note the controller provides similar control for this combination of vehicle positional changes as for the previous commanded positional changes (see figures 4.21-4.27) and for another set of positional changes depicted in figures 5.4-5.10 (ignoring the imposed disturbance). Thus the controller is shown to be equally effective no matter what the combination of coordinated maneuvers in the depth and trackline planes. 

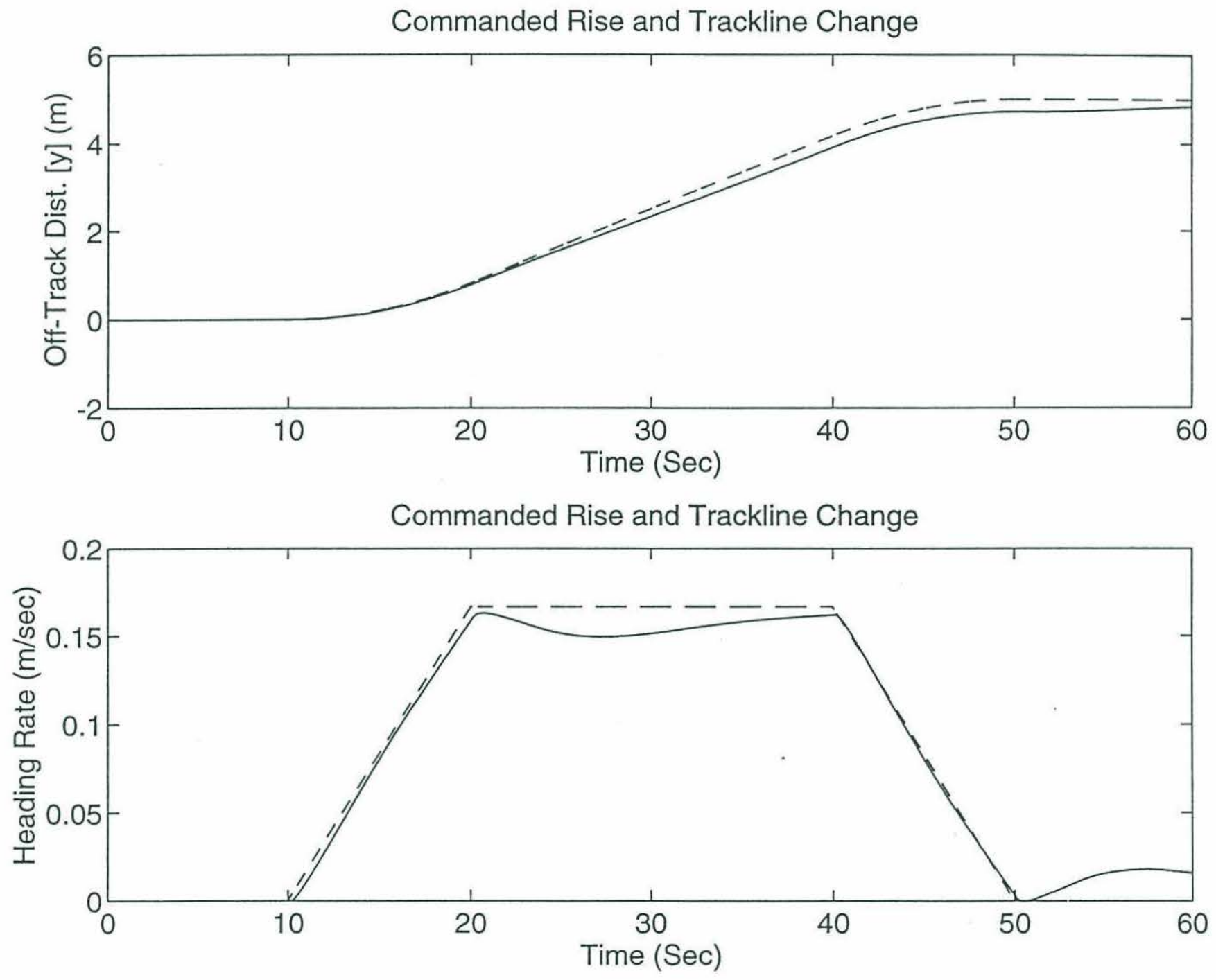

Figure 4.29 - Trackline and Heading Rate for a Coordinated Rise and Trackline Maneuver with a $10 \mathrm{~N}$ Positive Buoyancy. The $4 \mathrm{DOF}$ implementation of the $6 \mathrm{DOF}$ controller provides excellent control in this coupled maneuver. 

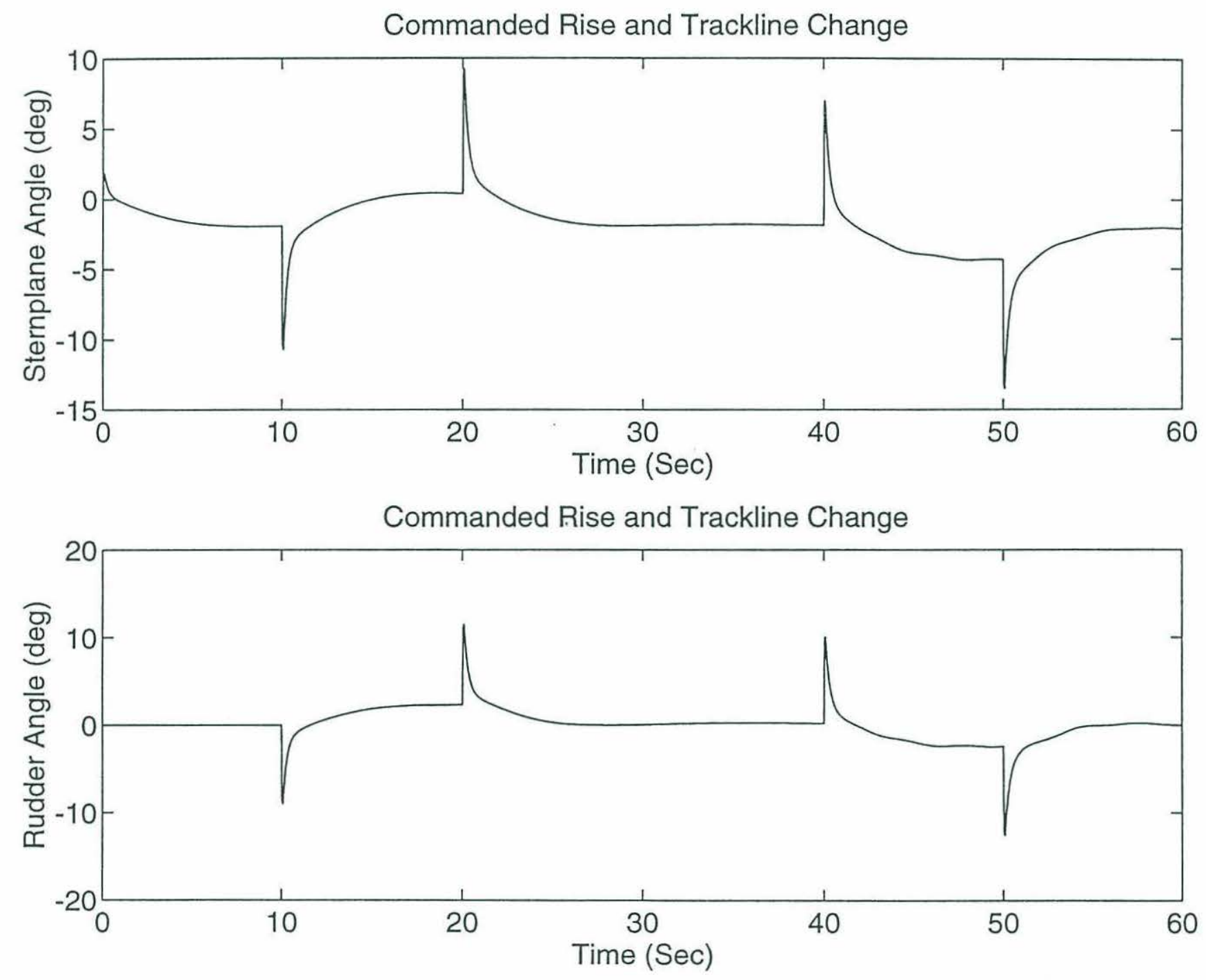

Figure 4.30 - Sternplane and Rudder Responses for a Coordinated Rise and Trackline Maneuver with a $10 \mathrm{~N}$ Positive Buoyancy. As expected from the symmetry about the $\mathrm{y}$ - and $\mathrm{z}$-axes, control surface actions are similar. Note the non-minimum phase actions of the control surfaces - first get the requisite pitch and yaw angles, respectively, then allow for the depth and trackline changes. 

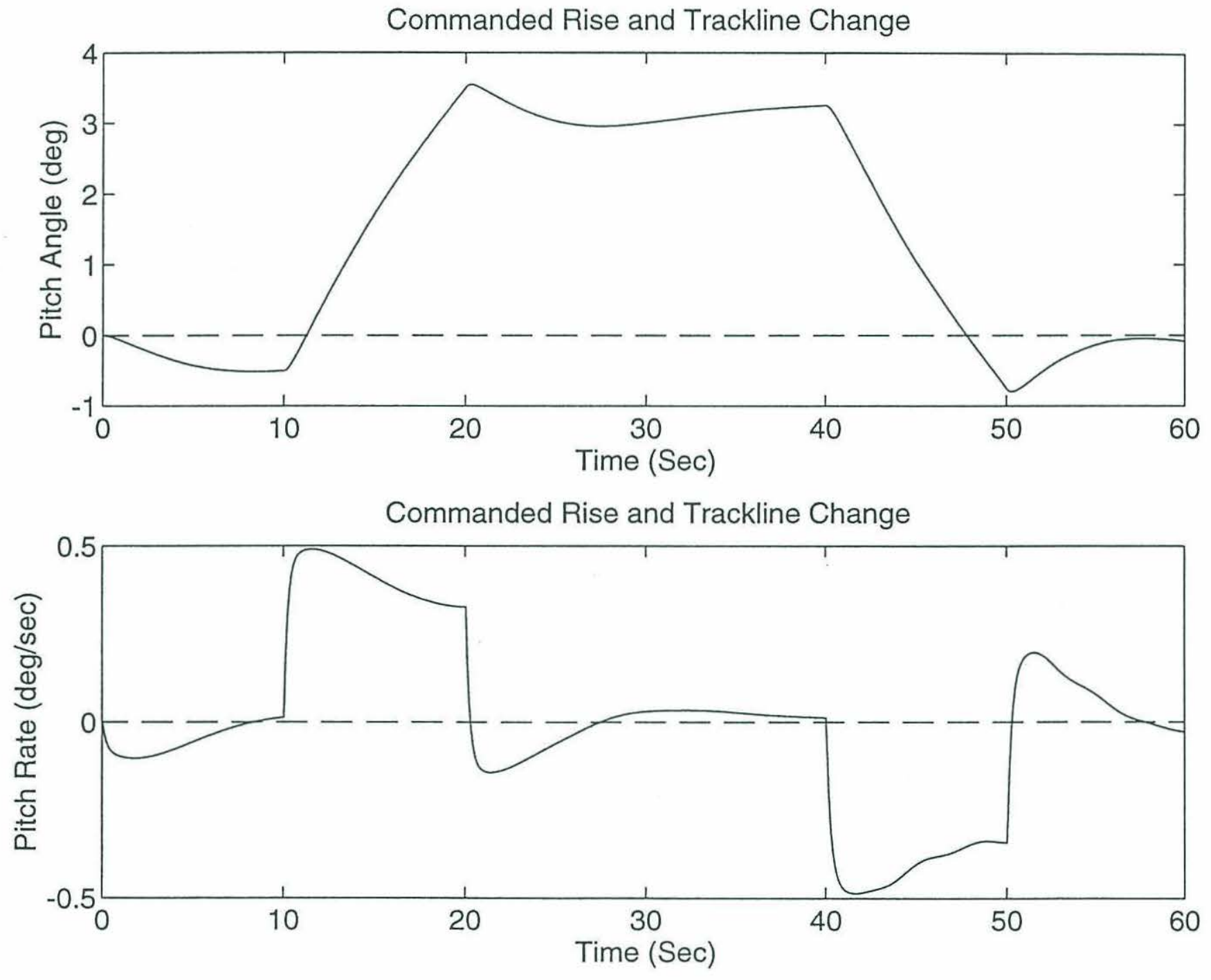

Figure 4.31 - Pitch and Pitch Rate for a Coordinated Rise and Trackline Maneuver with a 10 N Positive Buoyancy. Corresponds to depth and depth rate responses. 

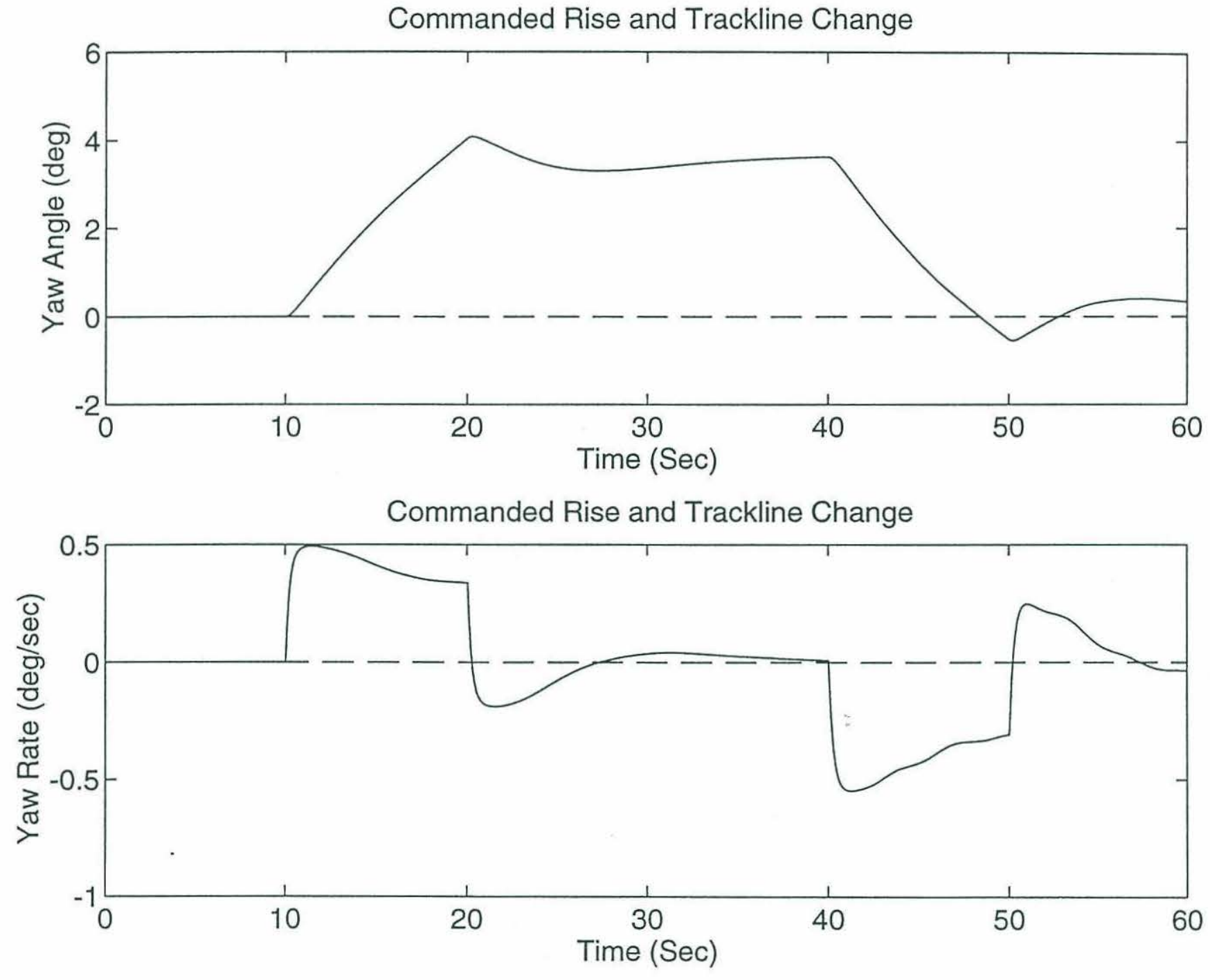

Figure 4.32- Yaw and Yaw Rate for a Coordinated Rise and Trackline Maneuver with a $10 \mathrm{~N}$ Positive Buoyancy. Corresponds to trackline and heading rate responses. 
Commanded Rise and Trackline Change
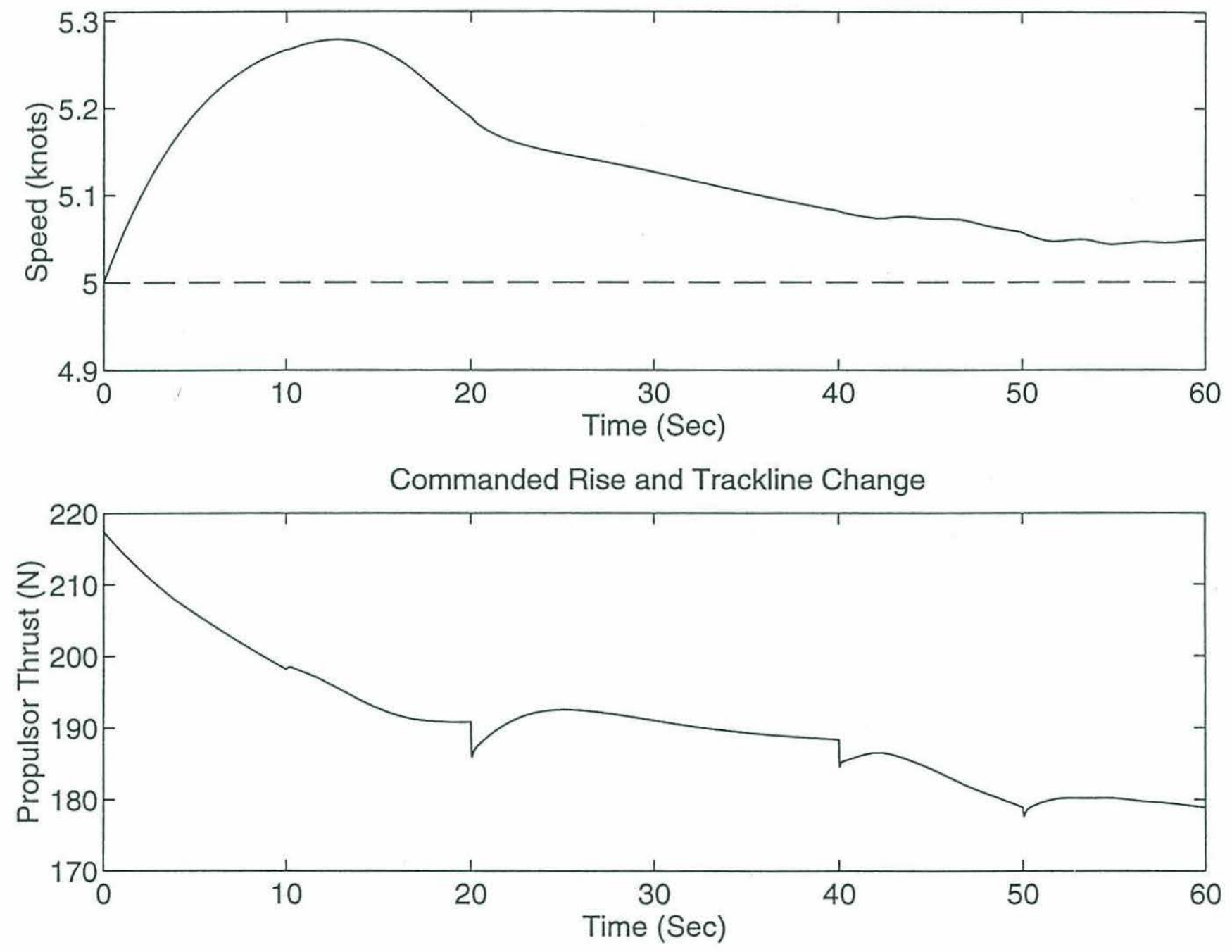

Figure 4.33- Forward Speed and Thrust for a Coordinated Rise and Trackline Maneuver with a $10 \mathrm{~N}$ Positive Buoyancy. Response as expected. 


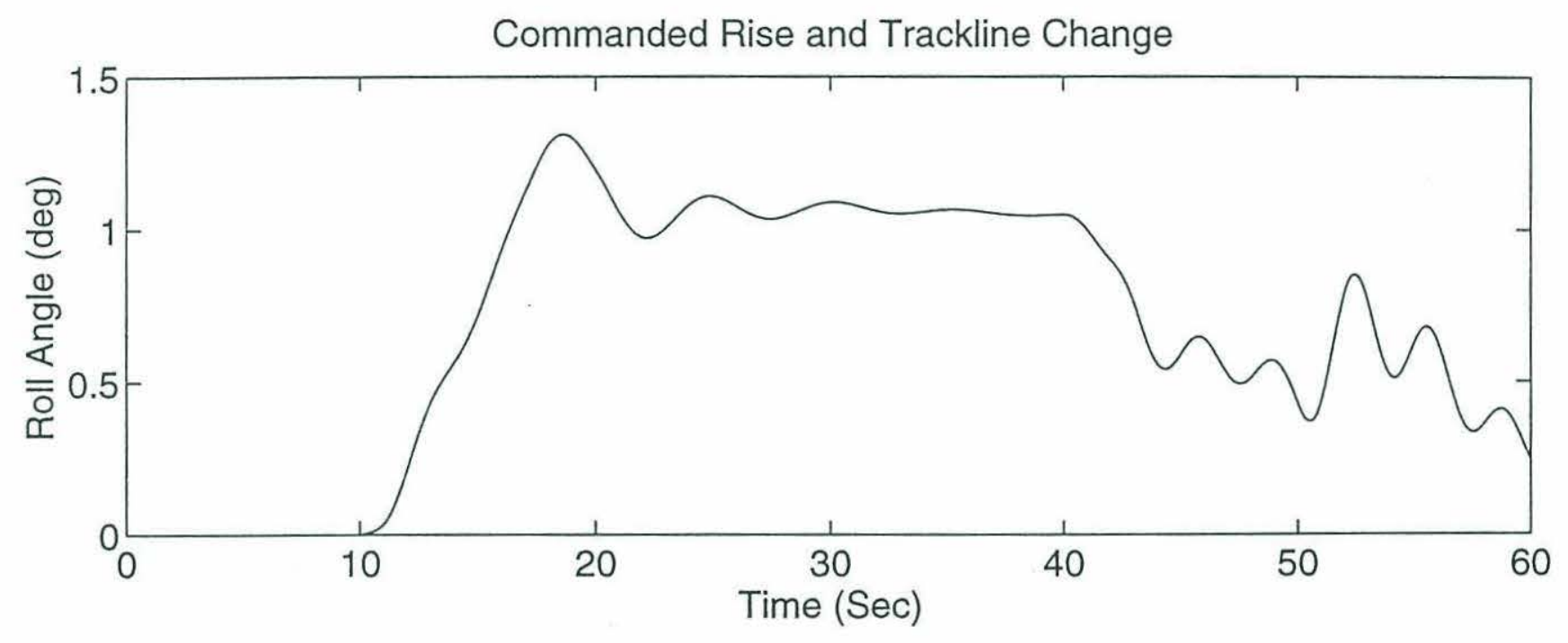

Commanded Rise and Trackline Change

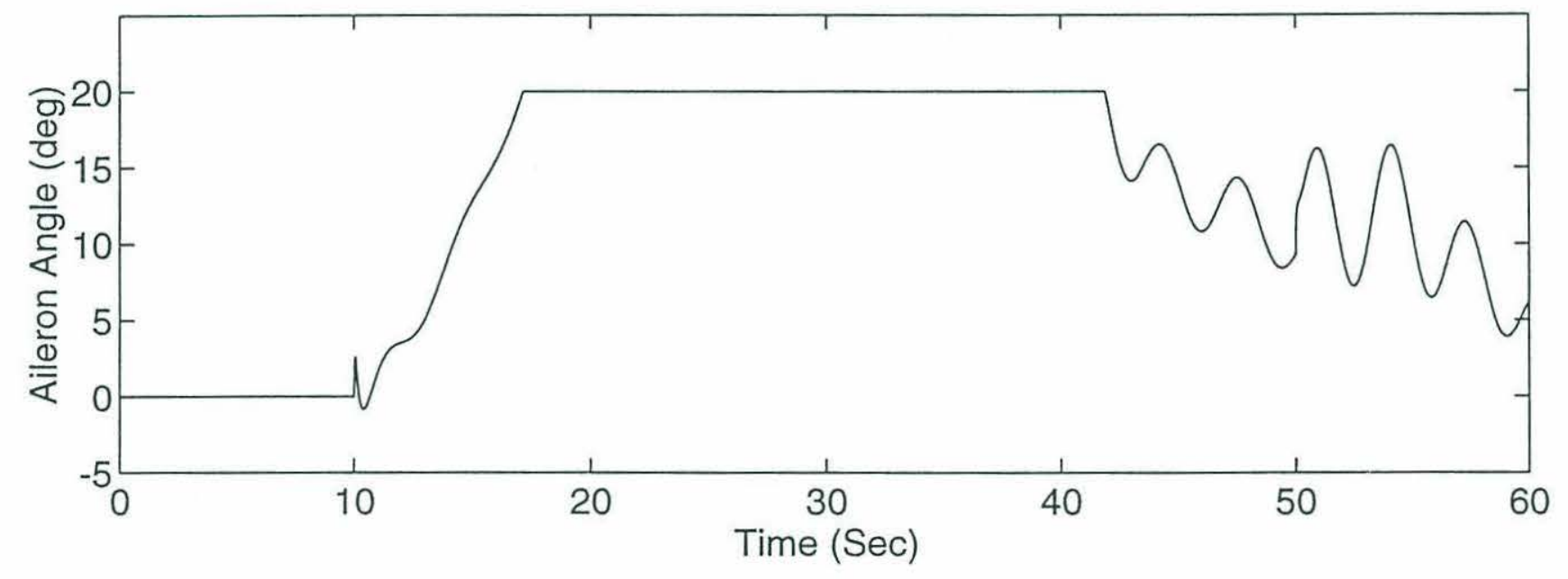

Figure 4.34- Roll and Aileron Angles for a Coordinated Rise and Trackline Maneuver with a $10 \mathrm{~N}$ Positive Buoyancy. See figure 4.17. 


\section{Chapter 5}

\section{Related Topics to the 6 DOF Sliding Controller}

In this chapter, two specific areas of UUV control that are facilitated by the framework of the 6 DOF sliding controller developed in Chapter 3 - variable buoyancy control and wave disturbance rejection - are briefly discussed accompanied by appropriate simulations. Directions further research in these areas might take is also discussed..

\subsection{Variable Buoyancy}

As the 6 DOF sliding controller operates, a vector consisting of the earthreferenced control forces/moments needed to achieve trajectory following is generated $\left(\tau_{\eta}\right.$ - see (3.15)). This signal is precisely what is needed for the input to a variable buoyancy controller which is trying to maintain the UUV in a neutrally buoyant condition!

Consider, for instance, the scenario depicted in figures 5.1-5.3 in which the 21UUV of figure 1.1 with the 4 DOF version of the 6 DOF sliding controller is commanded to travel a straight line at 5 knots. The vehicle is simulated to have $20 \mathrm{~N}$ positive buoyancy for the first portion of the "maneuver" which is then decreased to 10 
$\mathrm{N}$ positive buoyancy (e.g. the vehicle encounters a region of less dense water). As the vehicle orients itself to the weight/buoyancy mismatch, the $Z$ portion of $\tau_{\eta}$ approaches the negative value of this mismatch. This signal, perhaps passed though a low-pass filter to eliminate wave and other disturbances, is easily processed to control the requisite changing of the weight of the vehicle to restore neutral buoyancy.

Note how the 6 DOF sliding controller directly provides the signals needed for the task of buoyancy control. There is no need to correct for vehicle orientation with respect to the earth (it is already done), nor is their a need to resolve the vehicle control actions into the various DOF's as is necessary if trying to compute the weight/buoyancy mismatch from a measurement of the sternplane angle alone.

As mentioned, further work is needed get a viable buoyancy control system based on the 6 DOF sliding controller, mostly in the area of signal processing - e.g. filtering disturbances out of $\tau_{\eta}$, getting a good pitch commanded moment signal free of the 4 DOF feedthrough from the depth DOF etc. 


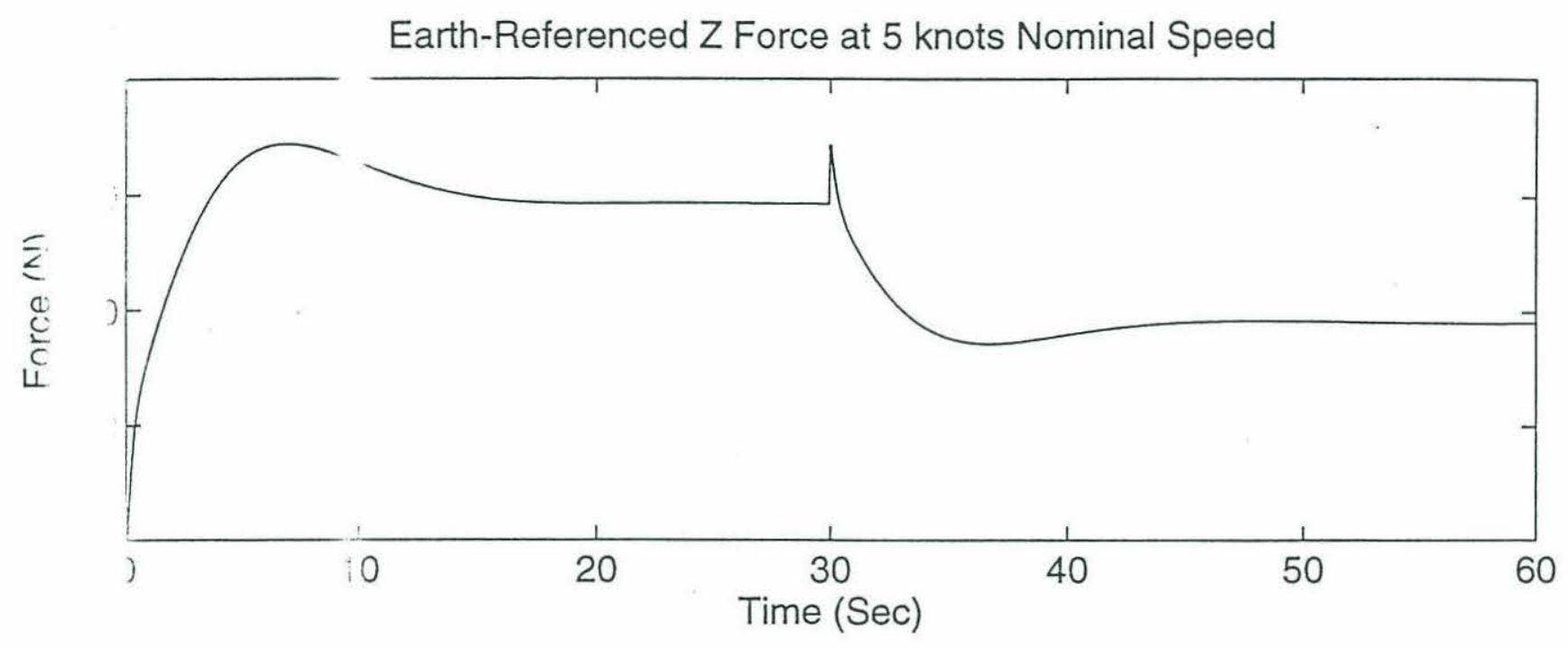

Earth-Referenced M Moment at 5 knots Nominal Speed

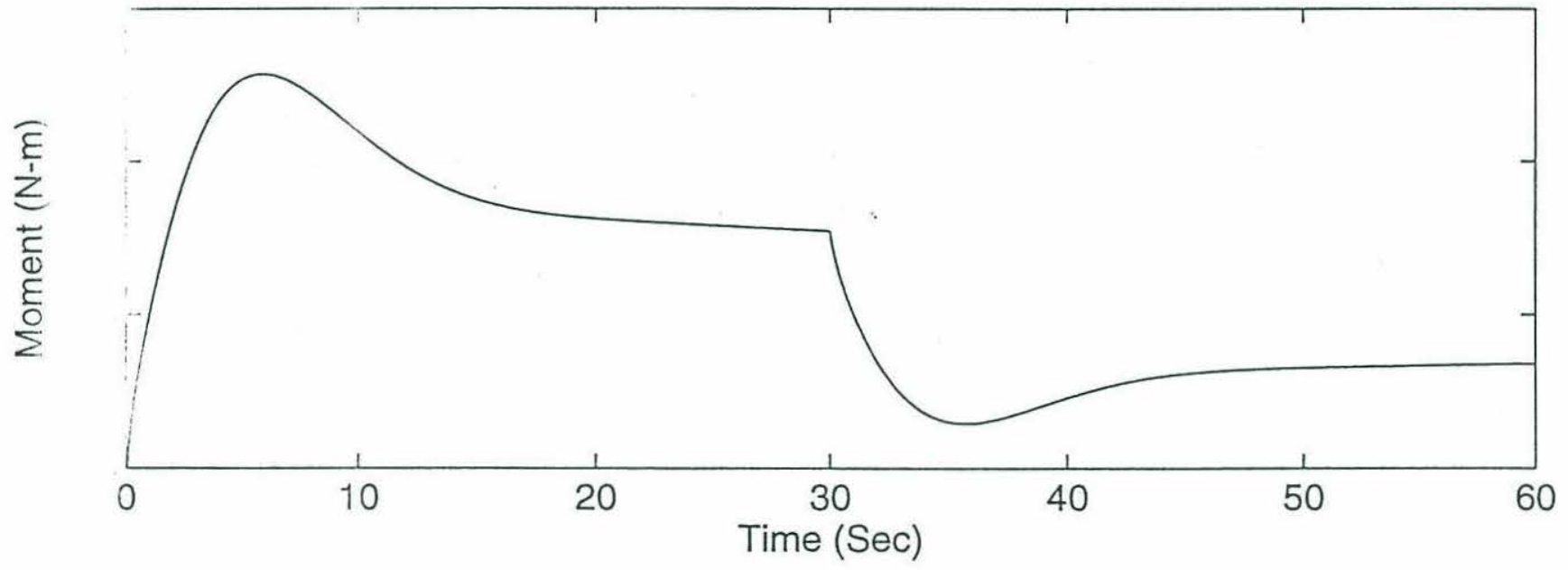

Figure 5.1 - 6 DOF Sliding Controller Generated Earth-Referenced Z Force and M Moment During a Step Change in Vehicle Buoyancy. The vehicle is travelling at 5 knots. Vehicle buoyancy initially assumed to be a positive $20 \mathrm{~N}$ and changed to (+) $10 \mathrm{~N}$ at 30 seconds (e.g. vehicle entered region of warmer water); $\mathrm{x}_{\mathrm{G}}$ assumed $.01 \mathrm{~m}$ forward of $\mathrm{x}_{\mathrm{B}}$ during the entire maneuver. These controller outputs are not colored by vehicle orientation - though for this particular scenario little error would be introduced by considering bodyreferenced forces and moments - and can be processed to provide control signals for a variable buoyancy system. The commanded moment is much larger than one would expect for the actual derivations from perfect trim - the depth buoyancy mismatch is feeding its way through the 4 DOF controller to this DOF. 


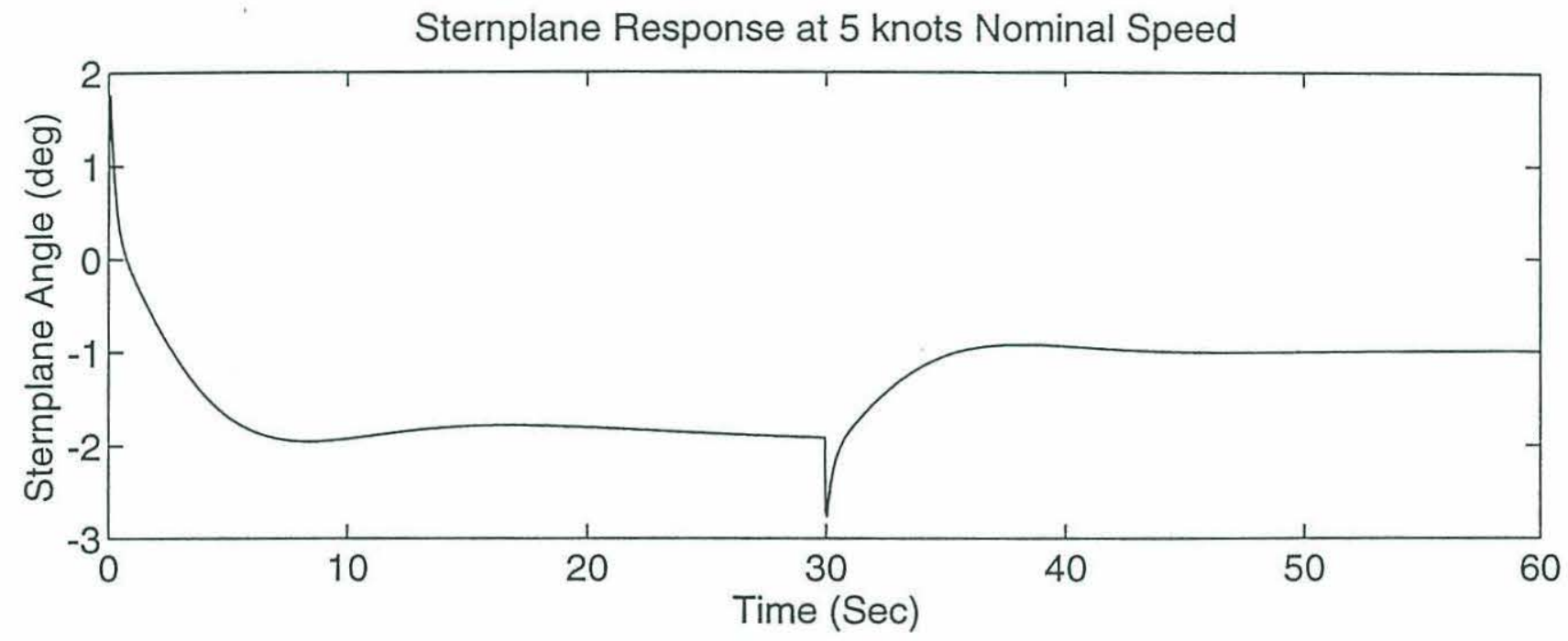

Figure 5.2 - Sternplane Response During a Step Change in Vehicle Buoyancy. Actually, the sternplane angle can be processed to provide control signals for a variable buoyancy system. However, vehicle orientation would have to be taken into account (though not a concern for this particular scenario) and the sternplane angle would somehow have to be split into a moment and force component. Why not just use the earth-referenced forces and moments already generated by the 6 DOF sliding controller? 

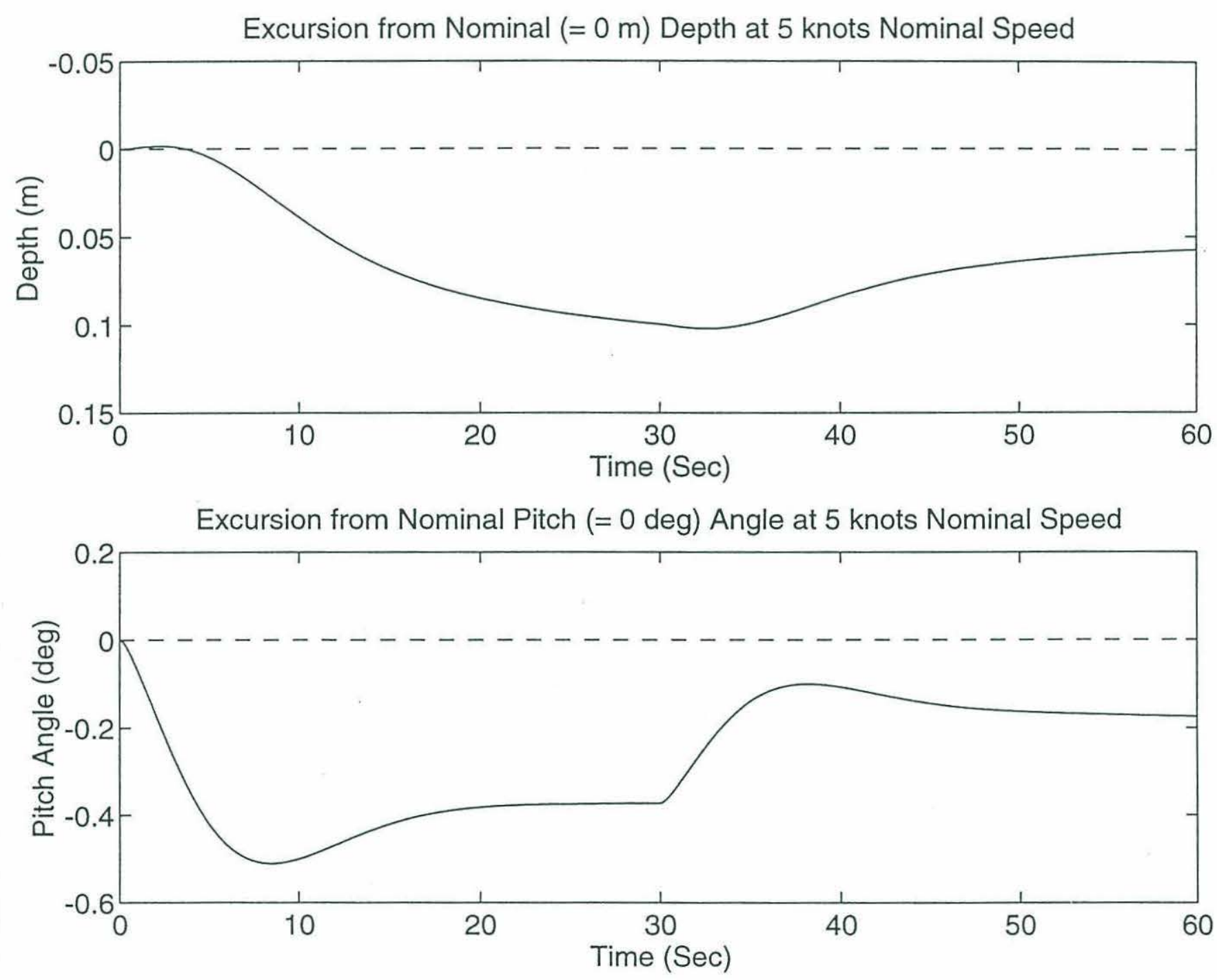

Figure 5.3 - Excursions from Nominal Depth and Pitch Angle During a Step Change in Vehicle Buoyancy. As expected the 4 DOF implementation of the 6 DOF sliding controller commanded control vector works well for this benign maneuver. 


\subsubsection{Disturbance Response}

A large motivating factor in the design of the 6 DOF sliding controller is to permit satisfactory vehicle operation in the dynamically-challenging regime of shallow water. As discussed, the direct accounting of the non-linearities and coupling inherent in the 6 DOF equations of motion by the controller that was designed in Chapter 3 allows the vehicle to withstand larger and more dynamically complicated disturbances than for simpler controller designs which largely ignore the coupling between the DOF's.

This is apparent in the simulation results depicted in figures 5.4-5.10 in which a coordinated trackline and depth change is performed in the presence of significant wave disturbances (assumed pitch/depth disturbance of 0.1 meters amplitude coupled with a yaw/heading disturbance of .05 meters amplitude at $.2 \mathrm{~Hz}$ ). The vehicle remains stable as it performs the maneuver though it does oscillate about its nominal orientation.

It is possible to reduce this oscillation by means of adaptive sliding control techniques (see Slotine and Li [1991] for instance). As mentioned in chapter 2, the force/moment vector $\tau$ can be viewed as consisting of a propulsion/control part and an environmental part. By assigning the proper structure to the environmental part - e.g. sinusoid at a known frequency but unknown amplitude - one can estimate the effect the wave field has on each DOF via an adaptive sliding controller which is fundamentally similar to sliding controller design. This is the topic of the sister study to this thesis Willy [1994]. We envision his single DOF adaptive sliding controllers being run in parallel with the $6 \mathrm{DOF}$ vehicle controller of this paper and providing the estimates of the

disturbances to the vehicle controller. This should significantly improve UUV 
performance in waves and warrants further analysis. 

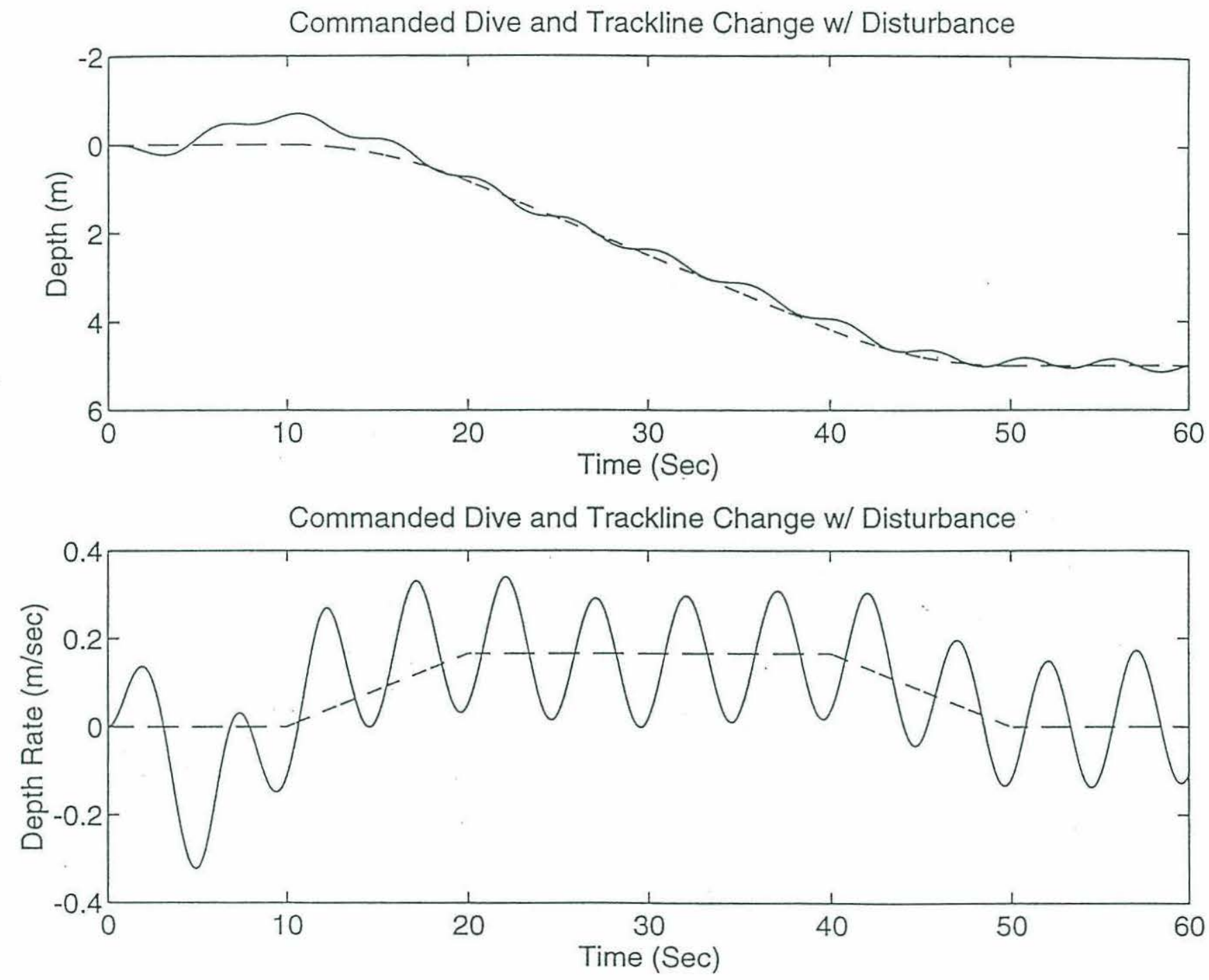

Figure 5.4 - Depth and Depth Rate for a Coordinated Dive and Trackline Maneuver with a $10 \mathrm{~N}$ Positive Buoyancy in the Presence of Simulated Wave Disturbances. The 4 DOF implementation of the 6 DOF controller provides excellent control in this coupled maneuver despite the significant imposed disturbance. 
Commanded Dive and Trackline Change w/ Disturbance

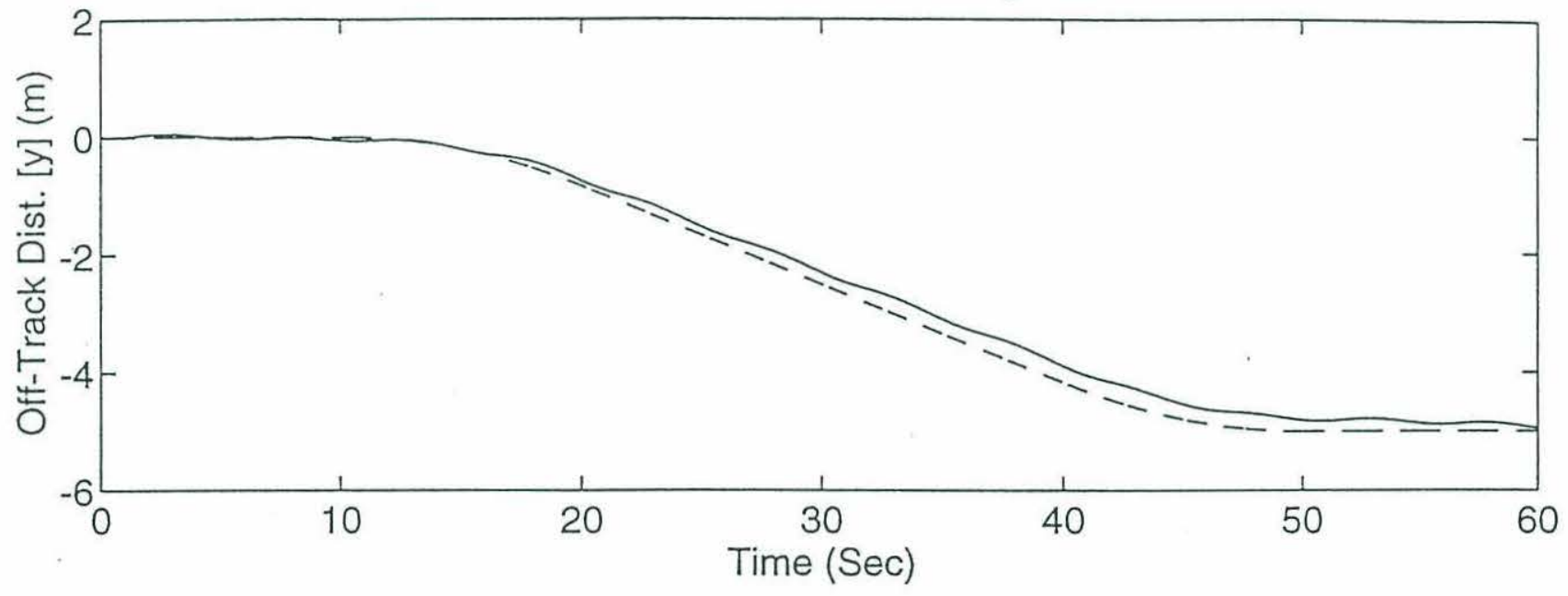

Commanded Dive and Trackline Change w/ Disturbance

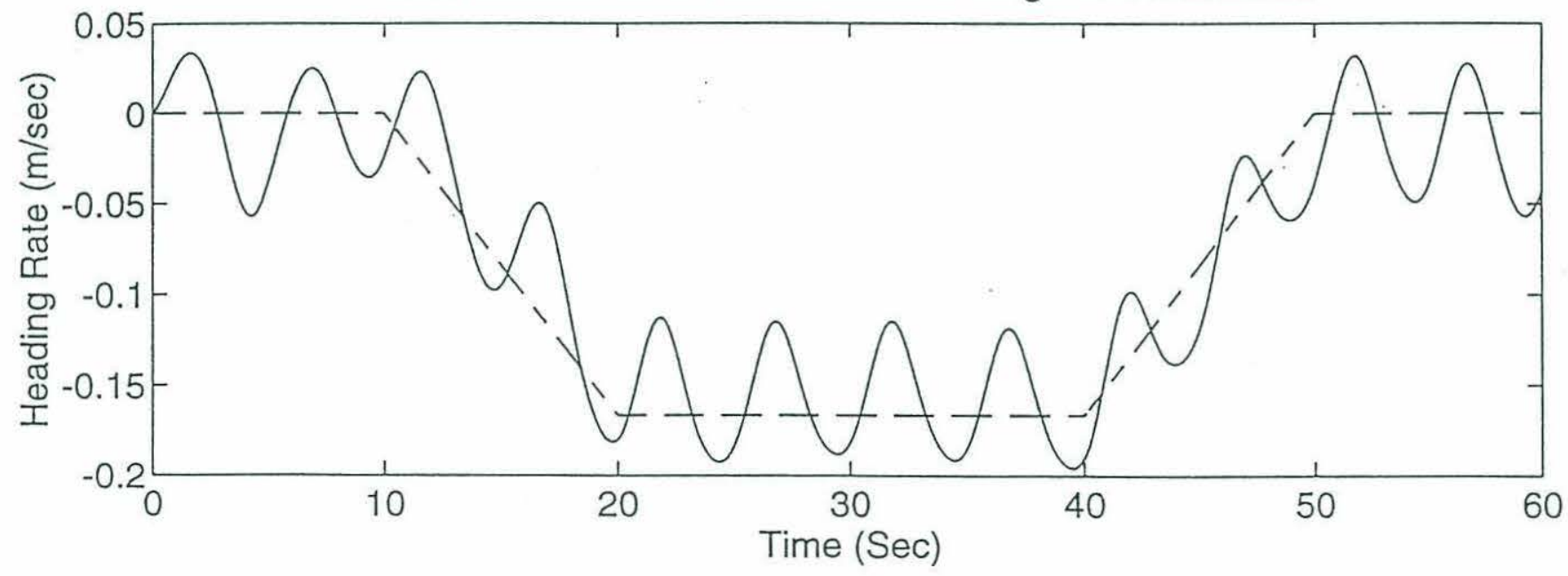

Figure 5.5 - Trackline and Heading Rate for a Coordinated Dive and Trackline Maneuver with a $10 \mathrm{~N}$ Positive Buoyancy in the Presence of Simulated Wave Disturbances. The 4 DOF implementation of the 6 DOF controller provides excellent control in this coupled maneuver despite the significant imposed disturbance. 


\section{Commanded Dive and Trackline Change w/ Disturbance}
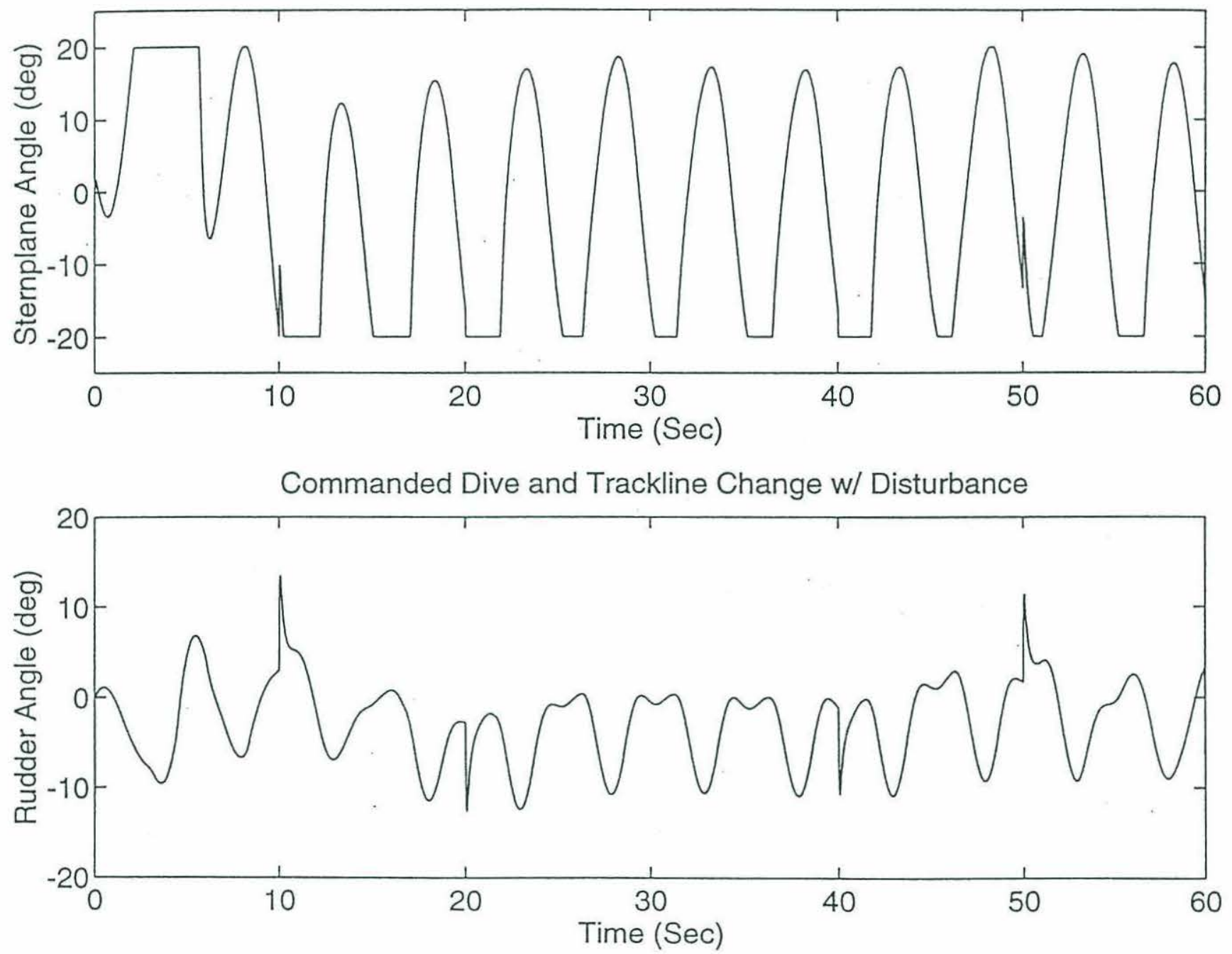

Figure 5.6 - Sternplane and Rudder Responses for a Coordinated Dive and Trackline Maneuver with a $10 N$ Positive Buoyancy in the Presence of Simulated Wave Disturbances. The imposed disturbance causes intermittent saturation of the sternplanes - the $y$-axis is not subjected to as high a disturbance magnitude, thus the rudders do not saturate - yet the vehicle controller is still able to maintain excellent trajectory following for this coupled maneuver as shown in figures 5.4 and 5.5. 

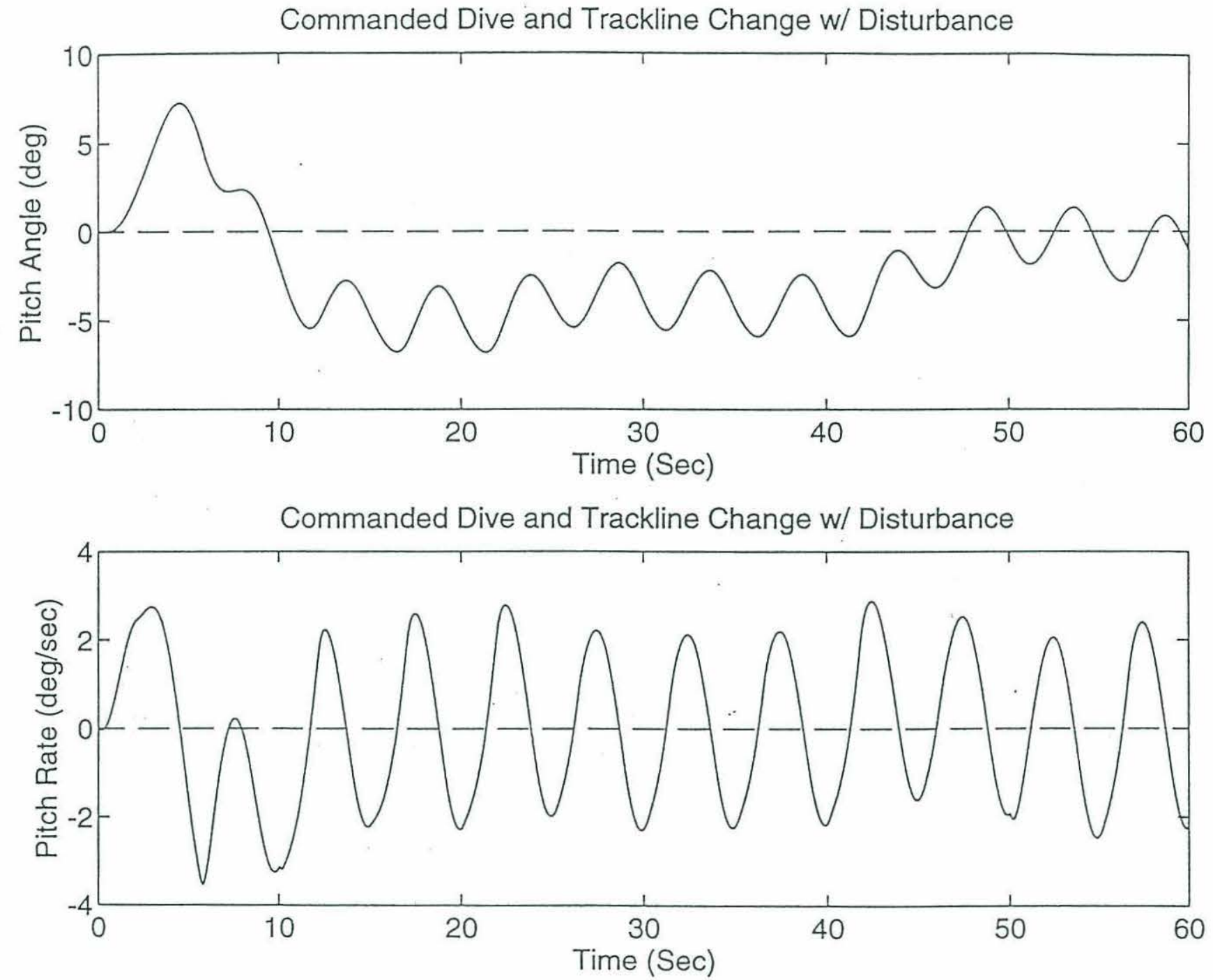

Figure 5.7- Pitch and Pitch Rate for a Coordinated Dive and Trackline Maneuver with a $10 \mathrm{~N}$ Positive Buoyancy in the Presence of Simulated Wave Disturbances. Again, despite the significant effect the disturbance has on the dynamics as shown here, the vehicle controller remains stable. 

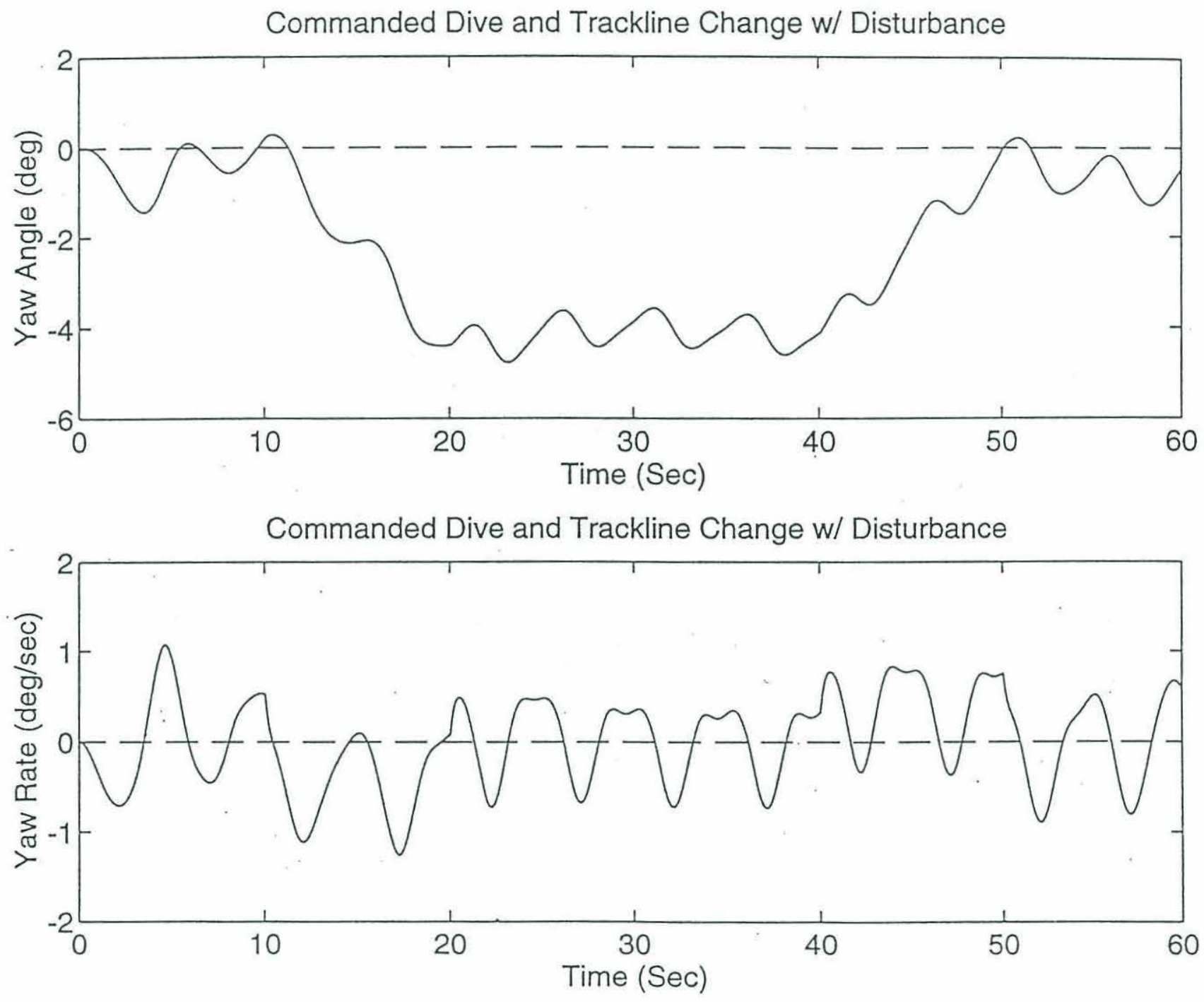

Figure 5.8- Yaw and Yaw Rate for a Coordinated Dive and Trackline. Maneuver with a $10 \mathrm{~N}$ Positive Buoyancy in the Presence of Simulated Wave Disturbances. See figure 5.7. 


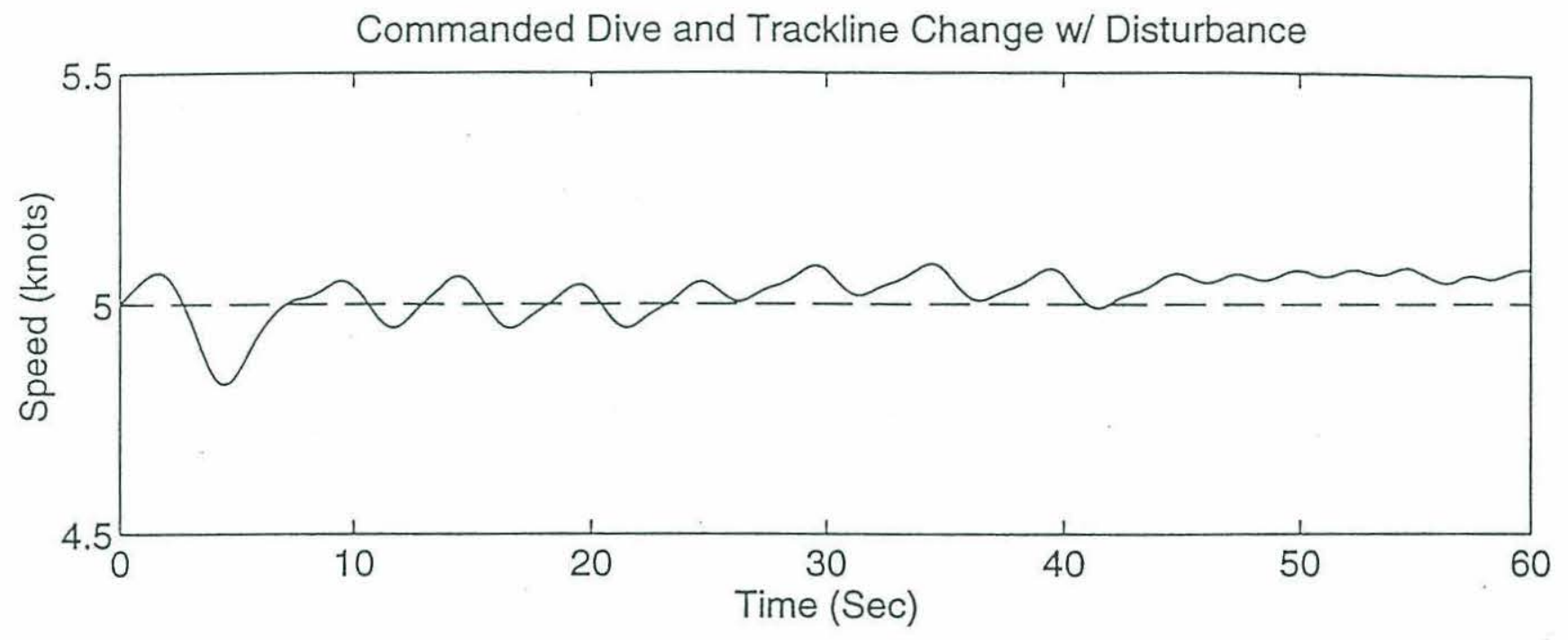

Commanded Dive and Trackline Change w/ Disturbance

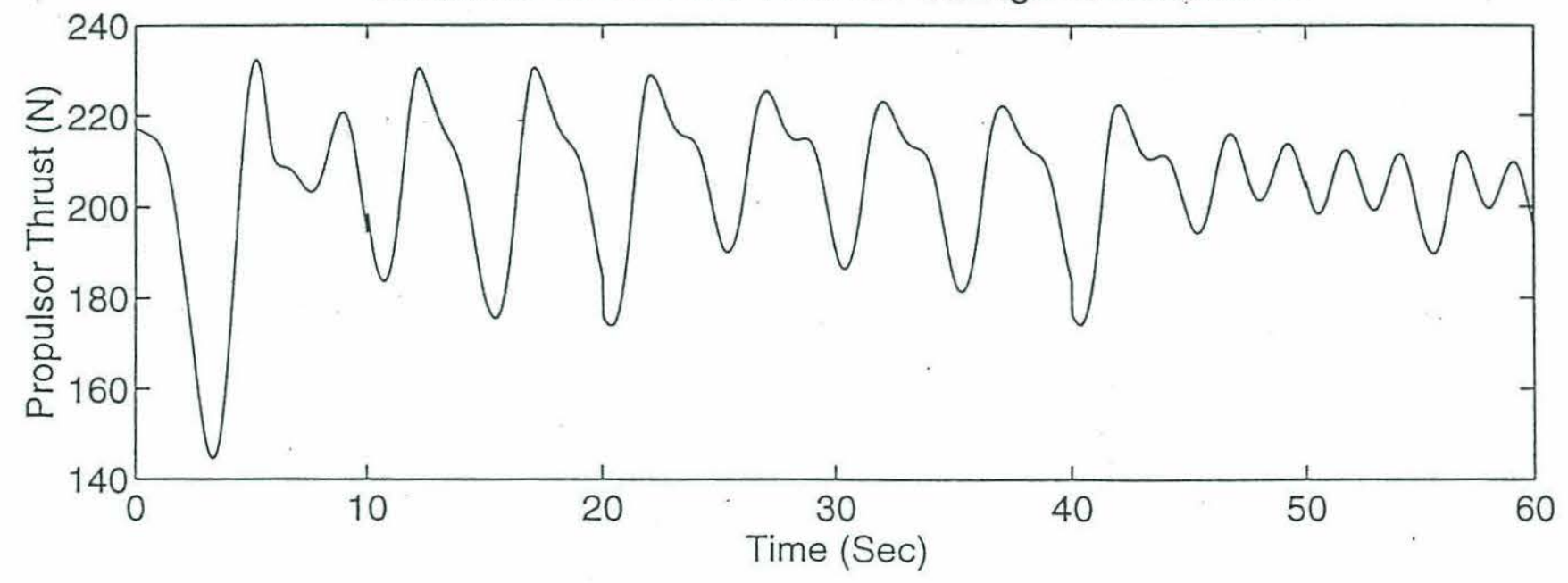

Figure 5.9- Forward Speed and Thrust for a Coordinated Dive and Trackline Maneuver with a $10 \mathrm{~N}$ Positive Buoyancy in the Presence of Simulated Wave Disturbances. The thrust response may not be realistically achievable. A better model of the propulsor is needed. 
Commanded Dive and Trackline Change w/ Disturbance

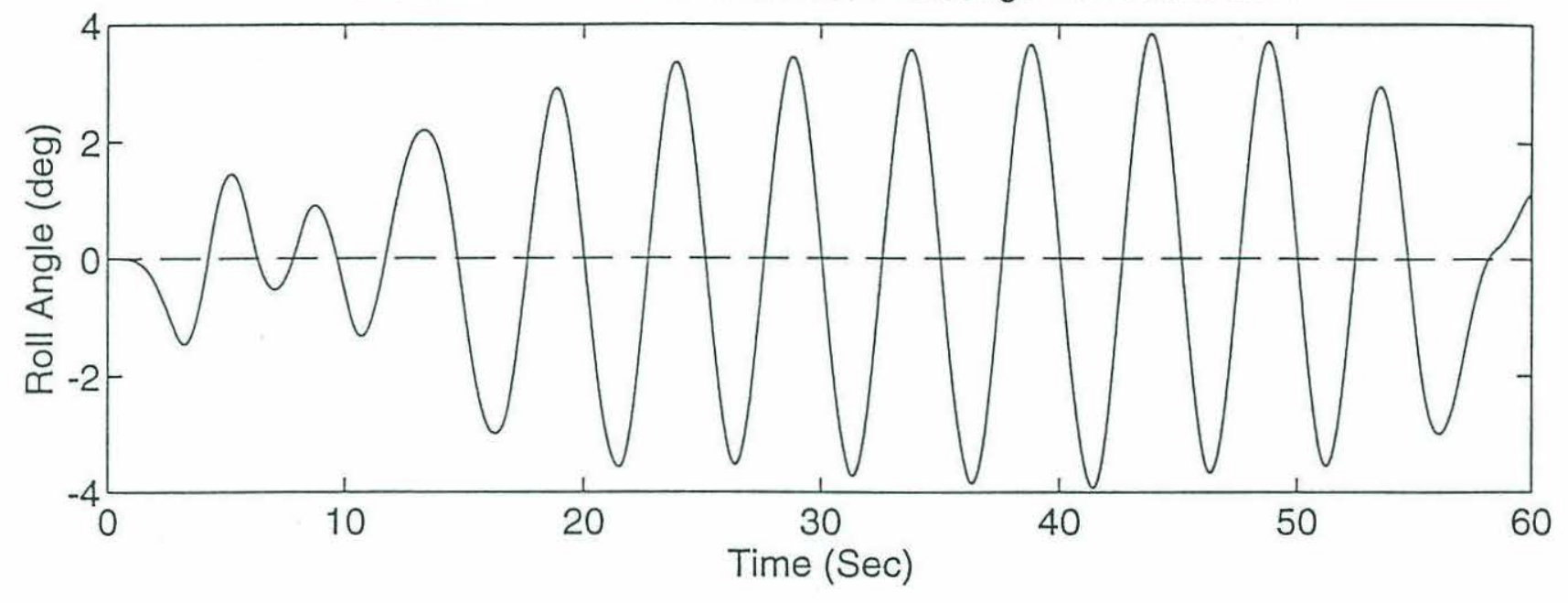

Commanded Dive and Trackline Change w/ Disturbance

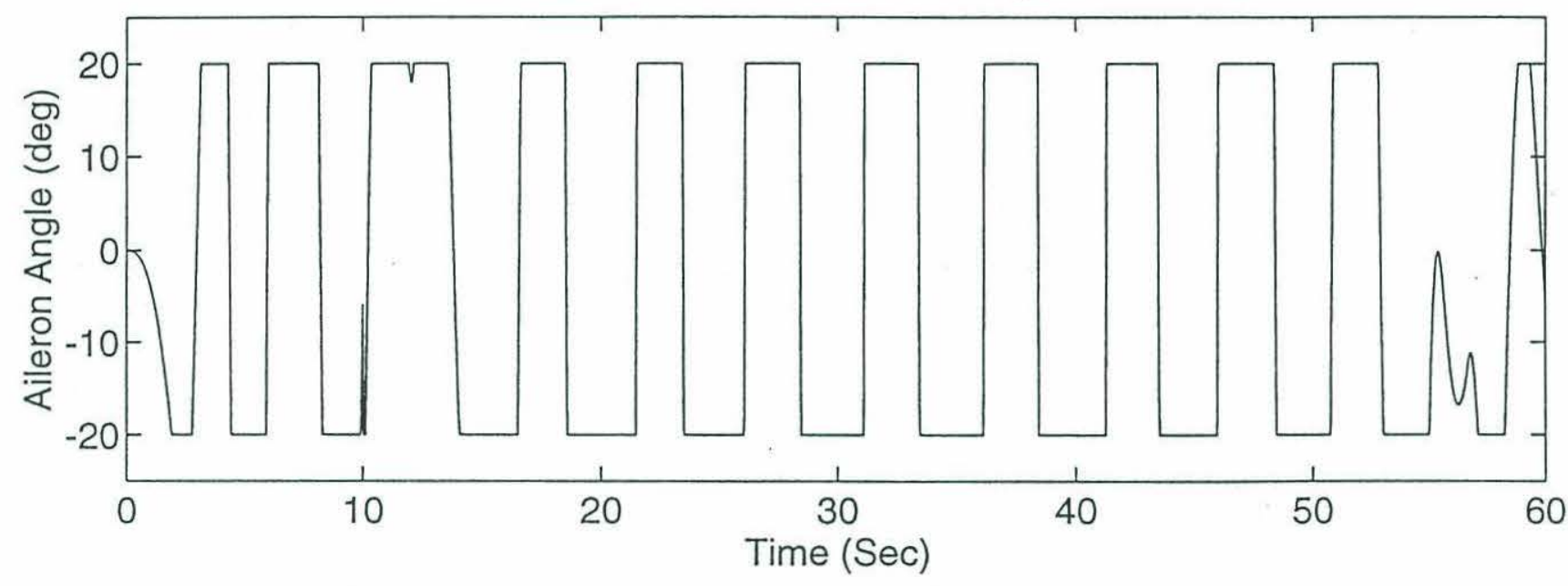

Figure 5.10- Roll and Aileron Angles for a Coordinated Dive and Trackline Maneuver with a $10 \mathrm{~N}$ Positive Buoyancy in the Presence of Simulated Wave Disturbances. Despite the near-constant aileron saturation, the roll response stays within reasonable bounds. Of course, this is primarily due to the sinusoidal nature of the disturbance and resultant sinusoidal yaw and pitch motion rather than by any control authority exerted by the ailerons (refer to figures 4.17 and 4.27). Matters could be worse for a constant disturbance affecting the roll DOF but it is difficult to imagine a natural phenomenon providing such a disturbance. For instance, a cross-current - though constant - can not really "catch" the control surfaces enough to cause the vehicle to start rolling as the force imposed on the upper control surfaces is counterbalanced by the force imposed on the lower ones. 


\section{Chapter 6}

\section{Conclusion}

A full accounting of the conclusions drawn from this research and suggested directions for continued investigation can be found in the preceding chapters. Section 6.1 provides an overview of the work summarizing the important results. Section 6.2 similarly summarizes the main areas where work remains to be done.

\subsection{Summary and Conclusions}

This study focused on the development of a six degree of freedom (6 DOF) robust sliding controller to be used on a variety of UUV designs in dynamically difficult regimes like shallow water as well as for complicated maneuvering such as hovering. As indicated, such operating conditions require full consideration of the non-linearities and coupling inherent in the UUV's equations of motion in addition to the parameter and structural uncertainties posed by these equations.

The hydrodynamic theory necessary for the development of this 6 DOF sliding controller was laid out in Chapter 2. Indeed, the main thrust of the development was the identification of a particularly useful structuring of the UUV equations of motion so that 
the non-linear control techniques used in Chapter 3 would result in an elegant and powerful controller. An important relationship between the vehicle- and earth-reference frames was established.

Chapter 3 formulated the robust sliding controller which was shown to be stable - assuming full 6 DOF control authority - from any vehicle configuration except at \pm 90 degrees of pitch.

As full 6 DOF control authority is usually not available, an attempt was made to develop a method to modify this sliding controller so that lesser degree of freedom vehicles (in terms of available control authority) would perform satisfactorily. As demonstrated with the 4 DOF 21UUV, this method - weighted least squares - is based heavily on the non-minimum phase relationship between pitch/depth and yaw/trackline.

Finally, in Chapter 5, two related control issues - buoyancy control and wave disturbance control/adaptation were briefly discussed and their connection with robust 6 DOF sliding control explored.

All developments in this study were extensively simulated, indicating that many of the ideas presented in this thesis have some practical use.

\subsection{Directions for Further Research}

Although a considerable amount of design effort has been expended in the development of the robust 6 DOF sliding controller, significant work remains to be done before it can be practically used on the 21UUV or similar vehicles. The body and hydrodynamic coefficients need to be as accurately computed as possible with good 
estimates of the bounds on their ranges. A dynamic model of the propulsor needs to be formulated and incorporated into the controller; dynamic models of the control surfaces can also be incorporated (or the $\Lambda$ matrix values in the sliding controller can be tuned down where appropriate).

If the UUV to be controlled is similar to the $21 \mathrm{UUV}$ in terms of control authority available (i.e. $4 \mathrm{DOF}$ ) then it is important to verify that the weightings in the weighted least squares methodology. If the controller is to be used for a vehicle designed to hover, then the $D$ matrix in the equations of motion should be extended to include those hydrodynamic terms that become important in this operating regime.

The variable buoyancy control ideas of Chapter 5 are easily implemented. Also, the disturbance rejection/adaptation ideas in this chapter need to be incorporated with the results of an adaptive controller developed in the sister study to this thesis - Willy [1994]. 


\section{References}

J. M. Anderson (1992). Unmanned Undersea Vehicle Model Analysis for Low Speed Controller Development. Naval Undersea Warfare Center Technical Memo. \#922087, Sept. 1992.

F. Dougherty and G. Woolweaver (1990). At-Sea Testing of an Unmanned Underwater Vehicle Flight Control System. In Symposium on Autonomous Underwater Technology, pp. 65-68, Washington DC.

O. M. Faltinsen (1990). Sea Loads on Ships and Offshore Structures. Cambridge University Press, New York.

T. I. Fossen (1991). Nonlinear Modeling and Control of Underwater Vehicles. Doctoral thesis, Division of Engineering Cybernetics, Norwegian Institute of Technology, Trondheim.

T.I. Fossen (1994). Guidance and Control of Ocean Vehicles. John Wiley and Sons, London.

S.J. Hills and D.R. Yoerger (1994). A Nonlinear Sliding Mode Autopilot for Unmanned Undersea Vehicles. To be published in Oceans '94 Proceeding, 1994.

D. E. Humphreys (1989). Hydrodynamic Design and Six-Degree-of-Freedom Maneuvering Evaluation of Naval Undersea Systems Center Unmanned Underwater Vehicle. A.R.A.P Report \#636, California Research \& Technology, Inc., McLean VA, March 1989.

S. I. Sagatun and T. I. Fossen (1991). Lagrangian Formulation of Underwater Vehicles' Dynamics. In Proceedings of the IEEE International Conference on Systems, Man and Cybernetics, pp. 1029-1034, Charlottesville, VA, October 13-16, 1991.

J. J. E. Slotine and W. Li (1991). Applied Nonlinear Control. Prentice-Hall Int., Englewood Cliffs, New Jersey 07632.

SNAME (1950). The Society of Naval Architects and Marine Engineers. Nomenclature for Treating the Motion of a Submerged Body Through a Fluid. In Technical and Research Bulletin No. 1-5.

G. Strang (1986). Introduction to Applied Mathematics. Wellesly-Cambridge Press, Wellesly, MA. 
K. Wendel (1956). Hydrodynamic Masses and Hydrodynamic Moments of Inertia. Technical Report. TMB Translation 260, July 1956.

C. J. Willy (1994). Attitude Control of an Underwater Vehicle Subjected to Waves. Engineer's/Master's thesis, MIT/WHOI Joint Program in Oceanographic Engineering.

D. R. Yoerger correspondence to S. Hills, Naval Underseas Warfare Center, Jan. 18,1994.

D. R. Yoerger, J. B. Newman and J. J. E. Slotine (1986). Supervisory Control System for the JASON ROV. IEEE Oceanic Engineering, OE-15, No. 3 pp. 167-179.

D. R. Yoerger and J. J. E. Slotine (1984). Nonlinear Trajectory Control of Autonomous Underwater Vehicles using the Sliding Methodology. In Proceedings of the ROV' 84 Conference, pp. 245-251.

D. R. Yoerger and J. J. E. Slotine (1985). Robust Trajectory Control of Underwater Vehicles. IEEE Oceanic Engineering, OE-10, No. 4 pp. 462-470. 


\section{Appendix I}

\section{UUV Body and Hydrodynamic Coefficients}

Body Coefficients - Assumed 5\% uncertainty

$\begin{array}{rlllll}\mathrm{W} & = & 15882 \mathrm{~N} & \mathrm{x}_{\mathrm{G}} & = & 2 \times 10^{-6} \mathrm{~m} \\ \mathrm{~B} & = & \text { varies } & \mathrm{y}_{\mathrm{G}} & = & 0 \mathrm{~m} \\ \mathrm{I}_{\mathrm{xx}} & = & 55.03 \mathrm{~kg}-\mathrm{m}^{2} & \mathrm{z}_{\mathrm{G}} & = & .005 \mathrm{~m} \\ \mathrm{I}_{\mathrm{yy}}=\mathrm{I}_{\mathrm{zz}} & = & 7100 \mathrm{~kg}-\mathrm{m}^{2} & \mathrm{x}_{\mathrm{B}}=\mathrm{y}_{\mathrm{B}}=\mathrm{z}_{\mathrm{B}} & = & 0 \mathrm{~m} \\ \mathrm{~m} & = & 1619 \mathrm{~kg} & & & \end{array}$

Hydrodynamic Coefficients - Assumed 20\% uncertainty

\begin{tabular}{|c|c|c|c|c|c|}
\hline $\mathrm{X}_{\mathrm{u}|\mathrm{u}|}$ & $=$ & $-33.69 \mathrm{~kg} / \mathrm{m}$ & $\mathrm{X}_{\delta \delta \delta s}=\mathrm{X}_{8 \mathrm{r} \delta \mathrm{r}}$ & $=$ & $-6.89 \mathrm{~kg} / \mathrm{m}$ \\
\hline $\mathrm{X}_{\mathrm{u}}$ & $=$ & $-41.5 \mathrm{~kg}$ & $\mathrm{X}_{\delta \mathrm{a} \delta \mathrm{a}}$ & $=$ & $-12.9 \mathrm{~kg} / \mathrm{m}$ \\
\hline$X_{\mathrm{wq}}=-\mathrm{X}_{\mathrm{vr}}$ & $=$ & $-1818 \mathrm{~kg}$ & $\mathrm{X}_{\mathrm{vor}}=-\mathrm{X}_{\mathrm{w} \delta \mathrm{s}}$ & $=$ & $5.25 \mathrm{~kg} / \mathrm{m}$ \\
\hline $\mathrm{Y}_{\mathrm{v}}=\mathrm{Z}_{\mathrm{w}}$ & $=$ & $-200 \mathrm{~kg} / \mathrm{m}$ & $\mathrm{X}_{\mathrm{r} \delta \mathrm{r}}=\mathrm{X}_{\mathrm{q} \delta \mathrm{s}}$ & $=$ & $-162 \mathrm{~kg}$ \\
\hline$Y_{r}=-Z_{a}$ & $=$ & $770 \mathrm{~kg}$ & $\mathrm{Y}_{\delta \mathrm{r}}=-\mathrm{Z}_{\delta \mathrm{s}}$ & $=$ & $11.5 \mathrm{~kg} / \mathrm{m}$ \\
\hline$Y_{i}=Z_{\dot{w}}$ & $=$ & $-1619 \mathrm{~kg}$ & $M_{\delta s}=N_{\delta r}$ & $=$ & $-355 \mathrm{~kg}$ \\
\hline$Y_{\dot{r}}=-Z_{\dot{q}}$ & $=$ & $-121.5 \mathrm{~kg}-\mathrm{m}$ & $\mathrm{K}_{\delta \mathrm{a}}$ & $=$ & $.556 \mathrm{~kg} / \mathrm{m}$ \\
\hline$M_{w}=-N_{v}$ & $=$ & 849 kg & $\mathrm{M}_{\mathrm{r} \delta \mathrm{a}}$ & $=$ & $-17.2 \mathrm{~kg}$ \\
\hline$M_{q}=N_{r}$ & $=$ & -3066 kg-m & $\mathrm{M}_{\mathrm{v} \delta \mathrm{a}}$ & $=$ & $.556 \mathrm{~kg} / \mathrm{m}$ \\
\hline$M_{\mathrm{w}}=-\mathrm{N}_{\mathrm{v}}$ & $=$ & $-121.5 \mathrm{~kg}-\mathrm{m}$ & $\mathrm{N}_{\mathrm{q} \delta \mathrm{a}}$ & $=$ & $14.7 \mathrm{~kg}$ \\
\hline $\mathrm{M}_{\dot{q}}=\mathrm{N}_{\dot{i}}$ & $=$ & $-7255 \mathrm{~kg}-\mathrm{m}^{2}$ & $\mathrm{~N}_{\mathrm{w} \delta \mathrm{a}}$ & $=$ & $.080 \mathrm{~kg} / \mathrm{m}$ \\
\hline
\end{tabular}

Notes: Notation of SNAME [1950] is used; for example, $\mathrm{X}_{\mathrm{u} \mid \mathrm{u}}$ is the coefficient for the term $X_{u|u|}|u|$ in the matrix $\boldsymbol{D}(v)$ of (2.23).

All control surfaces are assumed to have \pm 20 degree range of motion.

The propulsor is not dynamically modelled as its design (for the 21UUV) is largely unknown. Assumed a "perfect" thruster - delivers with no time delays what is commanded. 


\section{Appendix II}

\section{Proof of the Stability of the 6 DOF Sliding Controller}

A Lyapunov-like analysis using Barbalat's Lemma is performed to demonstrate the asymptotic stability of the 6DOF controller of Chapter 3 from any point in the vehicle state-space (except for the singularity at pitch angles of \pm 90 degrees). For a more complete treatment of Lyapunov stability theory, the reader is referred to texts such as Slotine and Li [1991].

From (3.8), the Lyapunov function chosen for the underwater vehicle equations of motion in the controller design is:

$$
V(t)=1 / 2 s^{T} M_{\eta} s>0
$$

with time derivative from (3.19):

$$
\dot{V}(t) \leq\left(-\sum_{i=1}^{6} \epsilon_{i}\left|s_{i}\right|-s^{T} D_{\eta} s\right) \leq 0
$$

The latter relationship implies that $V(\mathrm{t})<V(0)$ and, thus, $\boldsymbol{s}$ is upper bounded since $\boldsymbol{M}_{\eta}$ is positive definite. From (3.5) $\tilde{\eta}$ and $\tilde{\eta}$ are bounded and since the desired trajectories $\eta_{d}, \dot{\eta}_{d}$ and $\ddot{\eta}_{d}$ are bounded the reference trajectories $\eta_{r}$ and $\dot{\eta}_{r}$ and the actual trajectories $\eta$ and $\dot{\eta}$ are bounded from (3.6).

Combining the system dynamics of (2.26) with (3.5) yields:

$$
M_{\eta}\left(\dot{s}+\ddot{\eta}_{r}\right)+\left(C_{\eta}+D_{\eta}\right) \dot{\eta}=\tau_{\eta}-g_{\eta}
$$

Thus $\dot{s}$ is bounded since all the matrices (except at pitch angles of \pm 90 degrees) and vectors are bounded, and from (3.5) it follows $\ddot{\eta}$ must also be bounded. This implies that $\dot{V}(\mathrm{t})$ is bounded.

Application of Barbalat's Lemma then leads to the conclusion that $V(\mathrm{t}) \rightarrow 0$ as $\mathrm{t} \rightarrow \infty$, which implies that the tracking error $s$ and thus $\tilde{\eta}$ and $\dot{\tilde{\eta}}$ converge to zero. 